\title{
Theoretical Astrophysics in the Byurakan Observatory (1946 - 2021)
}

\author{
A.G. Nikoghossian* \\ NAS RA V. Ambartsumian Byurakan Astrophysical Observatory (BAO), Byurakan 0213, Aragatzotn Province, Armenia
}

\begin{abstract}
The purpose of this review is to give a general idea of the results on theoretical astrophysics investigations carried out at the Byurakan Observatory during the period of its existence. A special place in the review is given to the research work in the Department of "Theoretical Astrophysics", founded in 1965. The review consists of two parts. The first part is mainly devoted to works on the theory of radiation transfer somehow related to Ambartsumian's ideas developed in his fundamental studies of the 40-50s of the last century. The second part presents, on the one hand, the results which are of importance for theoretical physics in general and, on the other hand, gives their application to the interpretation of observational data of various cosmic objects. In order to get an idea of the importance of each result, the background and prerequisites of the research are given, indicating its significance from the point of view of astrophysics. Naturally, the most important results are presented in more detail. At the same time, the review in one way or another includes almost all of the most important results obtained by various members of the department at different times. It should be noted that works on some other areas of theoretical astrophysics are not included in the review, since they are presented in other reviews of this series.
\end{abstract}

\section{THEORY OF RADIATIVE TRANSFER}

\section{INTRODUCTION. PREREQUISITES.}

The middle of the last century was marked by the rapid development of radiation transfer theory, which was increasingly being used in a wide variety of astrophysical research fields. Along with classical theory, which dates back to the works of its founders Schuster, Milne, Eddington in connection with modeling the stellar atmospheres, new more advanced and flexible methods, better adapted to numerical calculations, appeared. Around these years, when the Byurakan observatory was founded, Ambartsumian's fundamental works appeared (Ambartsumian, 1941; 1942a,b; 1943a,b; 1944a,b,c; 1947; see also his Scientific Papers, 1960), which developed new ideas and approaches to solving various, in particular, classical problems of the radiative transfer. Since then, the mentioned theory with variety of its applications have always been one of the main directions of theoretical studies in the observatory. The following is a comprehensive description of the observatory's achievements in this and adjacent areas.

The study of the equation of radiation transfer and its solutions in various forms occupied important place in the first works aimed at interpreting the stellar spectra. Of course, there were investigated first of all simplest and therefore the rough models, in which the medium assumed to be plane-parallel, stationary, homogeneous and purely absorbing. The last assumption substantially simplifies the problem of finding the field of radiation in the atmosphere, since in this case the state of the radiating gas obeys the equilibrium laws of Saha and Boltzmann with the local values of temperature and density. In this approximation, referred to as the approximation of LTE (local thermodynamic equilibrium), the source function, appeared in the transfer equation, is given by the Kirchhoff-Planck law. The situation changes drastically, when one takes into account the scattering of radiation which is of particular importance in the problem of the spectral line formation. Now the state of the radiating gas depends not only on the local values of thermodynamic parameters, but also on the field of radiation at the particular point, which establishes coupling between different volumes inside the atmosphere. The equation of radiation transfer in this case is integral-differential and its solution, in general, encounters great difficulties.

In spite of the rough assumptions, the first works in this direction in many respects contributed to the physical understanding of the studied processes and stimulated the development of theory. The approach, which was developed in them and became classical, consisted in finding the source function as a function of the depth in the atmosphere, which, in its turn, made it possible to determine the field of radiation in it. In the simplest cases of isotropic and monochromatic scattering the problem mathematically is reduced to the solution of an integral equation of Fredholm's

*nikoghoss@bao.sci.am 
type with the kernel, which is the exponential integral depended on the modulus of a difference of the arguments. For instance, in the simplest case of isotropic scattering the problem of finding the radiation field in a semi-infinite atmosphere illuminated by a beam of parallel rays at angle of $\arccos \varsigma$ is reduced, as it is known well, to the solution of the following integral equation

$$
S(\tau, \varsigma)=\frac{\lambda}{2} \int_{0}^{\infty} \operatorname{Ei}\left(\left|\tau-\tau^{\prime}\right|\right) S\left(\tau^{\prime}, \varsigma\right) d \tau^{\prime}+\frac{\lambda}{4} e^{-\frac{\tau}{\varsigma}}
$$

where $\lambda$ is the single-scattering albedo (or the probability of the photon re-radiation during elementary act of scattering) and $S$ is the source function. Its solution allows, in particular, determining the intensity of the radiation emerging from the atmosphere, i.e., the quantity, directly measured during the observations.

\section{THE PRINCIPLE OF INVARIANCE}

In contrast to conventional approach described above, Ambartsumian proposed the new method, named by him 'the principle of invariance', which allowed to find the outgoing intensity without the preliminary determination of the light regime at all depths in the atmosphere. By principle of invariance, he implies such transformation of the initial atmosphere, which does not influence the global optical characteristics of a medium (Ambartsumian, 1942a,b; 1943a,b, 1980). The application of principle significantly facilitates the solution of the problems of radiation transfer, revealing from the very beginning the structure of the desired solutions what, in its turn, is a great help in determining the field of radiation inside the atmosphere. As it was shown subsequently (see below, Sect. 5, and also (Nikoghossian, 1999), the principle of invariance is, in fact, a special case of the more general variational principle, connected with the translational transformation of optical depth.

The invariance principle was formulated for the first time by Ambartsumian (1943a) in treating the problem of diffuse reflection of light from a semi-infinite homogeneous atmosphere. Considerations underwent the principle based on the apparent fact that the addition to this medium a layer of infinitesimal optical thickness $\Delta \tau$, possessing the same properties as the original one, must not change its reflectivity. This thesis was referred by the author to as the principle of invariance. This implies that the total contribution of processes relevant to the added layer will be equal to zero.

The reflectance of a medium is characterized by the reflection function $\rho(\eta, \varsigma)$, where $\varsigma$ and $\eta$ are respectively the cosines of the angles of incidence and reflection. Contribution of the all other possible processes is of the higher order of smallness with respect to $\Delta \tau$ and may be ignored. Then in the simplest case of isotropic scattering the condition of invariance of the reflection function for the semi-infinite atmosphere can be written in the form

$$
(\eta+\xi) \rho(\eta, \xi)=\frac{\lambda}{2} \varphi(\eta) \varphi(\xi)
$$

where the function

$$
\varphi(\eta)=1+\eta \int_{0}^{1} \rho\left(\eta, \eta^{\prime}\right) d \eta^{\prime}
$$

is referred to as the Ambartsumian $\varphi$ - function. The last two equations imply that the function $\varphi$ satisfies the following functional equation

$$
\varphi(\eta)=1+\frac{\lambda}{2} \eta \int_{0}^{1} \frac{\varphi(\eta) \varphi\left(\eta^{\prime}\right)}{\eta+\eta^{\prime}} d \eta^{\prime}
$$

usually called the Ambartsumian equation. Eq. (2) shows that the reflectance $\rho(\eta, \varsigma)$ is expressed through a function of one variable and is a symmetrical function of its arguments. The quantity $\eta \rho(\eta, \varsigma) d \eta$ possesses a probabilistic meaning, namely, it gives the probability that the quantum incident on the medium in the direction $\varsigma$, will be reflected by it in the directional interval $(\eta, \eta+d \eta)$. In the same paper the principle of invariance was applied for solving the problem of the diffuse reflection and transmission for the medium of finite optical thickness. In this case the layer of infinitesimal optical thickness $\Delta \tau$ is added to one of boundaries while such layer is subtracted from the opposite side. For the reflectance $\rho(\eta, \varsigma)$ and diffuse part of transmittance $\sigma(\eta, \varsigma)$ this results (for convenience of the further discussion, we adopt here somewhat different notations)

$$
\rho(\eta, \varsigma)=\frac{\lambda}{2} \frac{\varphi(\eta) \varphi(\varsigma)-\psi(\eta) \psi(\varsigma)}{\eta+\varsigma}, \quad \sigma(\eta, \varsigma)=\frac{\lambda}{2} \frac{\psi(\eta) \varphi(\varsigma)-\varphi(\eta) \psi(\varsigma)}{\eta-\varsigma} .
$$

The auxiliary functions $\varphi(\eta)$ and $\psi(\eta)$ are determined from the following system of functional equations 


$$
\begin{gathered}
\varphi(\eta)=1+\frac{\lambda}{2} \eta \int_{0}^{1} \frac{\varphi(\eta) \varphi\left(\eta^{\prime}\right)-\psi(\eta) \psi\left(\eta^{\prime}\right)}{\eta+\eta^{\prime}} d \eta^{\prime}, \\
\psi(\eta)=\mathrm{e}^{-\frac{\tau_{0}}{\eta}}+\frac{\lambda}{2} \eta \int_{0}^{1} \frac{\psi(\eta) \varphi\left(\eta^{\prime}\right)-\varphi(\eta) \psi\left(\eta^{\prime}\right)}{\eta-\eta^{\prime}} d \eta^{\prime},
\end{gathered}
$$

where $\tau_{0}$ is the optical thickness of the medium. These functions also are called the Ambartsumian functions. It is clear that the reflectance and transmittance, as well as the functions $\varphi(\eta)$ and $\psi(\eta)$, depend also on optical thickness of the medium, nevertheless, for brevity, $\tau_{0}$ is not indicated explicitly among arguments. As has already been indicated, the starting point for the determination of the intensity of radiation outgoing from the medium is here not the equation of transfer, which allows finding the required quantity only after the regime of radiation is found for all depths in the atmosphere. It is obvious that, in view of the linearity of problem, knowledge of the functions of reflection and transmission makes it possible to determine the intensity of the outgoing radiation for any flux falling on the medium. On the other hand, formulas (2) and (5) give solution not only of one particular problem of diffuse reflection (for the semi-infinite medium) or the problem of diffuse reflection and transmission (for the finite medium). In fact, they make it possible to reveal the structure of the global optical characteristics of medium, as such, expressing in this case the unknown quantities through the functions of one variable. Approach itself in many respects contributed to the presence of a number of important relations connecting with each other different characteristics of the radiation field, in the problems of the radiative transfer theory most frequently encountered in the astrophysical applications. Some of them follow directly from invariance property. Such relationships were obtained in the different time by a number of the authors both abroad (Sobolev, 1963; Chandrasekhar, 1960; Preisedorfer, 1976; Rybicky, 1977; Ivanov, 1978) and in Byurakan observatory (Yengibarian \& Mnatsakanian, 1974; Nikoghossian, 1995, 1997, 1999; Krikorian \& Nikoghossian, 1996).

It should be noted that the relations (2), (4) were obtained by Ambartsumian earlier by another way in considering the scattering of light by the atmospheres of planets (Ambartsumian, 1942b). The way chosen in the mentioned work consists in the formal differentiation of the initial integral equation for the source function over the optical depth. From a purely mathematical point of view the way proposed is of large importance, since it shows, how the solution of the integral equation of the Fredholm-type with the difference kernel can be reduced to the solution of functional equation.

The idea on the invariant property of the global optical characteristics of the scattering and absorbing atmosphere with respect to the layer addition was employed in the problem of the radiation diffusion through the optically thick medium (Ambartsumian, 1941; 1944a). This research implies that the function $\varphi(\eta)$ admits a physical interpretation which concerns the angular distribution of the intensity of radiation transmitted through the optically thick atmosphere in the absence of true absorption. Various meanings can be ascribed to this function, of which the limb-darkening law for the Sun is one of astrophysical examples.

In various astrophysical problems one encounters, as it is known, the necessity to find the radiation field within the medium. An important advantage of the invariance principle is that knowledge of the intensities of radiation outgoing from a medium facilitates the solution of this problem essentially (see, e.g., Sobolev, 1959). For instance, being applied to Eq. (1), the invariance principle makes it possible to reduce the solution of this Fredholm-type integral equation to the solution of following Volterra-type equation for an auxiliary function $\Phi(\tau)$ related with the resolvent function of Eq.(1) (see, e.g., Sobolev, 1957)

$$
\Phi(\tau)=L(\tau)+\int_{0}^{\tau} L\left(\tau-\tau^{\prime}\right) \Phi\left(\tau^{\prime}\right) d \tau^{\prime}
$$

where the kernel-function

$$
L(\tau)=\frac{\lambda}{2} \int_{0}^{1} \varphi(\varsigma) e^{-\frac{\tau}{\varsigma}} \frac{d \varsigma}{\varsigma}
$$

is known well in the radiative transfer theory (Ivanov, 1969; Case \& Zweifel, 1967). An explicit expression for the function $\Phi(\tau)$ was obtained in (Minin, 1958). For illustration, we limited our consideration by the simplest case of monochromatic scattering, though the described picture and conclusions remain valid for the much more general statement of the transfer problem. From the pure mathematical point of view, principle of invariance may be considered as a way reducing the boundary-value problem usually formulated for the source function to the solution of the initial-value or Cauchy problem.

The invariance principle has played an important role in the theory of radiative transfer. It has been especially effective as applied to relatively complicated problems in radiative transfer theory. According to the idea suggested in 


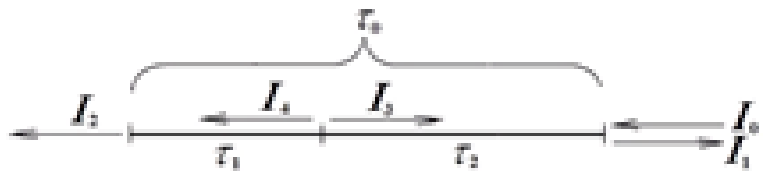

Figure 1: Schematic picture of the radiation transfer in two added layers

(Preisendorfer, 1976), this theory can be constructed so that it is based on the invariance principle, while the radiative transfer equation and the invariance relationships follow directly from it. Subsequently, as the theory developed, the feasibility of this approach became obvious and the direct use of this approach seemed preferable in some cases. The advantage of the approach lies in the profound intuitive content of the invariance principle and the existence of a close connection with the characteristic features of the physical problem under consideration, the symmetry property, and the boundary and initial conditions. In addition, as it is well known from physics, there is the relationship between invariance properties and conservation laws. In view of the importance of all these questions, they are examined them in more detail in Sect. 5 using radiative transfer in a plane-parallel atmosphere as an example.

\section{THE LAYERS ADDING METHOD}

As a result of Ambartsumian's studies in 1941-1948 on the theory of radiative transfer also another rather effective method, named by him the method of addition of layers, was proposed (Ambartsumian, 1944b). It gives response to the question, how the global optical characteristics of the absorbing and scattering media (coefficients of reflection and transmission) during their joining are added. It is obvious that this sufficiently general posing of the question appears naturally, if we abandon the requirement that the optical properties of a medium would remain unchanged during the addition to it of additional layer.

Because of importance of Ambartsumian's arguments in deriving the laws of addition of global optical characteristics for the further discussion, we present together with an explanatory figure the starting auxiliary equations written for the 1D homogeneous media. Fig. 1 shows a medium of optical thickness $\tau_{0}$ divided into two parts with thicknesses $\tau_{1}$ and $\tau_{2}$. Each of the media is characterized by reflection $r$ and transmission $q$ coefficients. The medium is illuminated externally and is supposed to be free of internal sources of energy. Based on some simple physical and probability arguments, one can write

$$
\begin{gathered}
I_{1}=r\left(\tau_{2}\right) I_{0}+q\left(\tau_{2}\right) I_{3}, \\
I_{2}=q\left(\tau_{1}\right) I_{4}, \\
I_{3}=r\left(\tau_{1}\right) I_{4}, \\
I_{4}=q\left(\tau_{2}\right) I_{0}+r\left(\tau_{2}\right) I_{3} .
\end{gathered}
$$

In view of the fact that $I_{1}=r\left(\tau_{1}+\tau_{2}\right) I_{0}$, and $I_{2}=q\left(\tau_{1}+\tau_{2}\right) I_{0}$, it is easy to obtain the requisite addition laws for the reflection and transmission coefficients of scattering and absorbing media

$$
\begin{gathered}
q\left(\tau_{1}+\tau_{2}\right)=\frac{q\left(\tau_{1}\right) q\left(\tau_{2}\right)}{1-r\left(\tau_{1}\right) r\left(\tau_{2}\right)}, \\
r\left(\tau_{1}+\tau_{2}\right)=r\left(\tau_{2}\right)+\frac{r\left(\tau_{1}\right) q^{2}\left(\tau_{2}\right)}{1-r\left(\tau_{1}\right) r\left(\tau_{2}\right)} .
\end{gathered}
$$

The quantities $q, r$ have a probabilistic meaning and may be correspondingly interpreted as the probabilities of the transmission and reflection of a photon incident on the medium.

Replacing $\tau_{2}$ by the infinitesimal $\Delta$ and passing to the limit when $\Delta \rightarrow 0$, one finds

$$
\begin{aligned}
\frac{\mathrm{d} q}{\mathrm{~d} \tau_{0}} & =-\left(1-\frac{\lambda}{2}\right) q\left(\tau_{0}\right)+\frac{\lambda}{2} q\left(\tau_{0}\right) r\left(\tau_{0}\right), \\
\frac{\mathrm{d} r}{\mathrm{~d} \tau_{0}} & =\frac{\lambda}{2}-(2-\lambda) r\left(\tau_{0}\right)+\frac{\lambda}{2} r^{2}\left(\tau_{0}\right) .
\end{aligned}
$$


The obtained system of non-linear differential equations satisfies the initial conditions $q(0)=1, r(0)=0$. Its solution is:

$$
r\left(\tau_{0}\right)=r_{0} \frac{1-\mathrm{e}^{-2 k \tau_{0}}}{1-r_{0}^{2} \mathrm{e}^{-2 k \tau_{0}}}, \quad q\left(\tau_{0}\right)=\left(1-r_{0}^{2}\right) \frac{\mathrm{e}^{-k \tau_{0}}}{1-r_{0}^{2} \mathrm{e}^{-2 k \tau_{0}}},
$$

where $k=(\lambda / 4)\left(1-r_{0}^{2}\right) / r_{0}$, and $r_{0}$ is the coefficient of reflection from a 1D semi-infinite atmosphere.

Thanks to their generality, these relations became a base for various modifications and stimulated the development of new methods in the radiative transfer theory. Some results obtained in the field are, in essence, nothing but elaboration of some special cases of the law of the layers addition. For instance, taking as one of the layers semi-infinite atmosphere and for another a layer of infinitesimal optical thickness, we are led to the problem considered in previous section, for which the invariance principle was formulated. When the infinitesimal layer is added to a finite layer, the requisite optical characteristics are found as the functions of optical thickness. Thus, the problem becomes 'imbedded' in a family of similar problems differing by the value of optical thickness. The generalization of this approach to the three-dimensional case was given by Chandrasekhar (1960). It underlies the method of invariant imbedding developed by Bellman and his co-authors $(1960,1963)$.

Finally, the method of addition of layers plays an important role in numerical solving the problems of radiation transfer in inhomogeneous atmospheres (see Sect.5.xvi). In this case the medium is divided into a number of layers in such a way as each of them can be regarded as homogeneous and the addition formulas (14), (15) are repeatedly applied (see, e.g., Bellman, Kalaba \& Wing, 1960; Bellman; Kalaba \& Prestrud, 1963; Van de Hulst, 1980; Nikoghossian, 2004). It is noteworthy that in the course of derivation of requisite optical parameters the fluxes appearing at the interfaces between adjacent layers are eliminated. Accordingly, in each application of the addition formulas one deals only with the intensities at the boundaries of the composite atmosphere. This is pointed out in some papers (Redheffer, 1962; Grant \& Hrant, 1968; Peraiah, 1999) in which the addition formulas are treated in the case when the component layers are allowed to be inhomogeneous. In the last two works the law of addition of layers is called "the star product".

\section{OTHER DIRECTIONS}

i. Statistical description of the radiation diffusion. In astrophysical problems, it is often necessary to estimate various statistical averages which describe the diffusion of radiation in an atmosphere. It is especially important to get some idea of the average number of scattering events underwent by a photon as a result of multiple scattering. In one of his early papers, Ambartsumian (1948) derived for this value the formula

$$
N=\lambda \frac{\partial \ln I}{\partial \lambda}
$$

where $I$ is the intensity of radiation. This formula served as a starting point for the study of statistical mean values describing the process of the radiation diffusion in the scattering and absorbing medium. It has been shown in a series of papers that the invariance principle offers an effective method for determining the mean number of scattering events, as well as the average time, for a photon travel in a medium. In particular, it was found that Eq. (19) is only valid for estimating the average number of scattering events for "moving" photons, i.e., those which have not been thermalized in the medium (see below Sect. 5.iv).

ii. Anisotropic scattering. The first works on the principle of invariance concern the monochromatic and isotropic scattering. It was apparent, however, that the principle may also be applied under much more general assumptions on the elementary act of scattering. That is why as early as in the paper Ambartsumian (1943b) handles the problem of diffuse reflection of light from a semi-infinite plane-parallel atmosphere for anisotropic scattering with arbitrary phase function. It is noteworthy that here he employs the expansion of the phase function $x(\gamma)$ ( $\gamma$ is the angle of scattering) in a series of Legendre polynomials which was suggested earlier by him (Ambatsumian, 1941, 1942a)

$$
x(\gamma)=\sum_{i=0}^{\infty} x_{i} P_{i}(\cos \gamma) .
$$

or with use of the summation theorem for spherical functions

$$
x\left(\eta, \eta^{\prime}, \varphi-\varphi^{\prime}\right)=\sum_{m=0}^{\infty} \cos m\left(\varphi-\varphi^{\prime}\right) \sum_{i=m}^{\infty} c_{i m} P_{i}^{m}(\eta) P_{i}^{m}\left(\eta^{\prime}\right),
$$

where the constants $c_{i m}$ are expressed through the coefficients $x_{i}$, and $P_{i}^{m}(\eta)$ are the associated Legendre polynomials. 
Then an expansion similar to (21) holds for the reflection function

$$
\rho\left(\eta, \eta^{\prime}, \varphi-\varphi^{\prime}\right)=\sum_{m=0}^{\infty} \rho_{m}\left(\eta, \eta^{\prime}\right) \cos m\left(\varphi-\varphi^{\prime}\right) .
$$

Now the solution of the problem is written by means of the functions $\varphi_{i}^{m}(\eta)$

$$
\rho_{m}\left(\eta, \eta^{\prime}\right)=\frac{\lambda}{4} \sum_{i=m}^{\infty}(-1)^{i+m} c_{i m} \frac{\varphi_{i}^{m}(\eta) \varphi_{i}^{m}\left(\eta^{\prime}\right)}{\eta+\eta^{\prime}},
$$

which are determined from the following set of functional equations

$$
\varphi_{i}^{m}(\eta)=P_{i}^{m}(\eta)+2 \frac{(-1)^{i+m}}{2-\delta_{0 m}} \int_{0}^{1} \rho_{m}\left(\eta, \eta^{\prime}\right) P_{i}^{m}\left(\eta^{\prime}\right) d \eta^{\prime},
$$

where $\delta_{k m}$ is the Kronecker symbol.

In the same paper these results were illustrated in treating two special cases: the scattering with two-term and the Rayleigh phase functions, which are of astrophysical importance.

iii. Non-LTE atmospheres. Since the mid-60s, the principle of invariance began to be applied to solve various problems of radiation transport of interest to astrophysics. One of directions was non-linear problems relating with the transfer in the Non-LTE atmospheres, in which the effect of the radiation field in the medium on the local optical properties of the medium is taken into account. These problems are extremely complicated but also important from an astrophysical standpoint. The nonlinear effect shows up even in the case of a single spectral line (Ambartsumian, 1964a,b,c, 1966, 1988, 1998). In these papers Ambartsumian studies the possibility of generalizing the layers adding method to non-linear problems. It was shown, in particular, that finding the intensity of radiation emerging from a finite medium reduces to solving a quasilinear partial differential equation that includes both the derivative with respect to the limiting optical thickness and the derivative with respect to the intensity of the flux incident on the medium.

A special place in the non-linear theory of radiative transfer is occupied by so-called multilevel problems, in which radiation is redistributed over different lines as it diffuses in a medium. One of the simplest problems this type was examined by Ambartsumian (1964a). It was assumed that the medium consists of three-level atoms, with transitions between the two lowest levels being neglected. It was found that depending on the magnitude of the fluxes incident on the medium, it can become transparent in one of the lines. The approach employed for this problem involved first finding the radiation field and, therefore, the degree of excitation in the medium in terms of the ordinary linear theory for an arbitrary value of the actual optical thickness and then finding the latter as a function of limiting optical thickness and of the incident radiation flux. Ambartsumian called this approach the method of self-consistent optical depths.

\section{DEPARTMENT OF THEORETICAL ASTROPHYSICS}

The department of theoretical astrophysics in the Byurakan observatory was established in 1965 to further develop the theory of radiation transfer and its applications in interpreting the spectra of various space objects.

i. Nonlinear problems. The first works in the department studied the possibility of generalizing the method of addition of layers and invariance principle to nonlinear problems in which the effect of the radiation field in the medium on optical properties of the medium becomes essential. The problems are highly important from an astrophysical standpoint since the effect shows up even in the case of a single spectral line, when the density of the radiation is relatively high and induced emission processes begin to play an important role (Ambartsumian, 1964a,b, 1966). In this relatively simple situation the problem mathematically becomes linear by proper replacement of variables. In the simplest case of pure scattering in homogeneous atmospheres, the usual expression $q=1 /[1+(\tau / 2)]$ for the transmission coefficient is replaced by

$$
q=\frac{1+\alpha F}{1+\frac{\tau}{2}+\alpha F}
$$

where $F$ is the intensity of the flux incident on the medium, $\alpha=(1 / a)\left(1+g_{2} / g_{1}\right), g_{1}$ and $g_{2}$ are the statistical weights of the upper and lower layers, respectively, and $a=8 \pi h v^{3} / c^{2}$ is the Planck factor, $\tau$ represents the limiting value of the optical thickness of the medium corresponding to the case when all the atoms lie in the lower state. The latter is introduced in nonlinear problems because the actual optical thicknesses depend on the radiation fluxes incident on the medium and are found after solving the problem. The method of self-consistent optical depths was used by Terebizh (1967) to solve two linearized transfer problems. An important result obtained in the area is that finding 
the intensity of the radiation emerging from a finite medium now reduces to solving a quasilinear partial differential equation that includes both the derivative with respect to the limiting optical thickness and the derivative with respect to the intensity of the flux incident on the medium. The further progress in the theory is connected with relatively recently published works by Pikichyan $(2010,2016 \mathrm{a}, \mathrm{b}, \mathrm{c}, 2019)$.in which an alternative way of linearization of the problems was proposed and some inverse problems were treated.

The so-called multilevel problems, in which radiation is redistributed over different lines as it diffuses in a medium, occupy an important place in the nonlinear theory of radiative transfer. As it was above said, the simplest problems of this type was examined by Ambartsumian (1964b, 1996), and also (1988, 1998). It was found that, depending on the magnitudes of the fluxes incident on the medium with three-level atoms, the medium can become transparent in one of the lines.

The three-level problem of diffuse reflection from a semi-infinite atmosphere under more general assumptions particularly when all the transitions between energy levels are permitted has been examined using the invariance principle by Nikoghossian $(1964,1965)$. The problems were reduced to the solution of a partial quasilinear differential equations, the first integrals of which were found analytically. Later, the same problems, however, with use of the method of addition of layers were considered by Gros \& Magnan (1981) and Magnan (1993) who developed a convenient numerical procedure for the solution. The three-level problem in the Schwarzschild-Shuster approximation for pure scattering was considered by Terebizh (1969).

ii. Homogeneous atmosphere. An important place in investigations of the department occupied an analytical work aimed at developing new schemes for the solution of classical problems of radiation transfer, facilitating the obtaining of the required solutions numerically. With limited computing capacity in the early years of the department, such work became particularly important. At the initial stage, relatively simple problems concerning mainly isotropic scattering of radiation in a homogeneous atmosphere were considered.

For instance, Danieian (1976) showed that the internal field of radiation in the finite medium illuminated by the parallel beam of rays can be found without integration over the optical depth. Approximate analytic solutions were derived by Mnatsakanian $(1975,1976)$ for determining the internal field of radiation in the medium of finite optical thickness under assumption that scattering is monochromatic and isotropic. Although these solutions are essentially asymptotic, in terms of the optical thickness of the medium, they are accurate within a few percent for the media of any thickness.

Due to the effectiveness of Ambartsumian's methods in the mentioned classes of problems, numerical algorithms for calculating fundamental $\varphi$ - function for semi-infinite media, as well as $\varphi$ - and $\psi$ - functions for atmospheres of finite optical thickness, were developed (Danielian \& Pikichyan, 1977; Andreasian \& Danielian, 1978, 1983). Thus, for example, in the last of these works, a new scheme of numerical calculation of the $\varphi$-function was proposed, which has a relatively high convergence rate. A special place in the research was occupied by issues related to the study of properties and the definition of ways to facilitate the determination of Green's function (Pikichyan, 1978a, 1980). The Green function approach in the non-coherent transfer problems was considered in (Pikichyan, 1978b). The specific properties of homogeneous atmospheres were used by Pikichyan (1982a,b; 1983, 1984) to treat media of arbitrary geometrically and physical characteristics. The explicit expression for the resolvent function for the problem of radiation transfer in an atmosphere with horizontally homogeneities was obtained by Danielian (1989).

The structure of the functions characterizing the non-stationary field of radiation has been studied by Terebizh (1968). It was shown that the similarity principle allows to reduce the solutions of the vast class of problems to the solution for a special case for only one value of the scattering albedo.

iii. Anisotropic scattering. Following Ambartsumian's approach to the problems of anisotropic scattering in its general formulation was treated by Nikoghossian \& Harutyunian (1978). The asymptotic behavior of Ambartsumian functions $\varphi_{i}^{m}(\eta)$ for large values of indices is employed in elaborating a simplified method of solving the problem of diffuse reflection of radiation from a semi-infinite atmosphere. The method was exemplified on the Henyey-Greenstein phase function

$$
x(\gamma)=\frac{1-g^{2}}{\left(1+g^{2}-2 g \cos \gamma\right)^{3 / 2}},
$$

where $g$ is a parameter that determines the lengthening of the phase function. There was estimated the rate at which the functions $\varphi_{i}^{m}(\eta)$ approach to spherical functions $P_{i}^{m}(\eta)$ for large values of $n$, as well as, the dependence of the rate of convergence to true values of the reflection function on the degree of the phase functions elongation.

iv. Partial redistribution over frequencies and directions. It is well known that the multiple scattering of the line radiation in various astrophysical media undergoes redistribution over frequencies and directions. The transfer problem arisen in the general case of partial redistribution is similar in many respects to that for anisotropic scattering. This analogy is especially distinct when one uses the bilinear expansions of the redistribution functions. Thus, for 
example, in the simplest case of a pure Doppler redistribution $r_{1}$ in Hummer's numeration (Hummer, 1962; Mihalas, 1970) it has been shown by Nikoghossian (1977) that the expansion

$$
r_{\mathrm{I}}\left(x^{\prime}, x, \gamma\right)=\frac{1}{\sqrt{\pi} \sin \gamma} \exp \left\{\frac{x^{2}+x^{\prime 2}-2 x x^{\prime} \cos \gamma}{\sin ^{2} \gamma}\right\}=\sum_{k=0}^{\infty} \cos ^{k} \gamma \alpha_{k}(x) \alpha_{k}\left(x^{\prime}\right)
$$

holds, where $x$ and $x$ ' are the so-called dimensionless frequencies of the incident and scattered photons measured from the center of the spectral line in units of the Doppler width and

$$
\alpha_{k}(x)=\left(2^{k} \sqrt{\pi} k !\right)^{-1 / 2} \exp \left(-x^{2}\right) H_{k}(x)
$$

is an orthonormal system of functions with weight $\exp \left(x^{2}\right)$ expressed in terms of Hermite polynomials, $H_{k}(x)$. Analogous bilinear expansion was obtained in the same paper also for the case of the combined Doppler and damping effects $\left(r_{I I I}(x, x, \gamma)\right)$, the latter being due to radiation and collision.

$$
r_{I I I}\left(x^{\prime}, x, \gamma\right)=\frac{\sigma \csc \gamma}{\pi^{3 / 2} U(0, \sigma)} \int_{-\infty}^{\infty} \mathrm{e}^{-u^{2}} \frac{U(x \csc \gamma-u \operatorname{ctg} \gamma, \sigma \csc \gamma)}{\left(x^{\prime}-u\right)^{2}+\sigma^{2}} \mathrm{~d} u,
$$

where $U(x, \sigma)$ is the Voigt function with the parameter $\sigma$, Note that when $\sigma \rightarrow 0, r_{\mathrm{III}} \rightarrow r_{\mathrm{I}}$. The proper bilinear expansion has a form

$$
r_{\mathrm{III}}\left(x^{\prime}, x, \gamma\right)=\frac{1}{U(0, \sigma)} \sum_{k=0}^{\infty} \cos ^{k} \gamma \alpha_{k}\left(x^{\prime}, \sigma\right) \alpha_{k}(x, \sigma)
$$

where

$$
\alpha_{k}(x, \sigma)=\frac{\sigma}{\pi} \int_{-\infty}^{\infty} \frac{\alpha_{k}(u) \mathrm{d} u}{(x-u)^{2}+\sigma^{2}}=(-1)^{k}\left(2^{k / 2} \pi^{1 / 4} \sqrt{k !}\right)^{-1} \frac{\mathrm{d}^{k} U(x, \sigma)}{\mathrm{d} x^{k}} .
$$

It is easy to see that the case $\gamma=0$ corresponds to coherent scattering, while $\gamma=\pi / 2$ - to the scattering with complete redistribution.

For directionally averaged redistribution laws, there was obtained

$$
r_{\mathrm{I}}\left(x^{\prime}, x\right)=\int_{\max \left(|x|,\left|x^{\prime}\right|\right)}^{\infty} \exp \left(-u^{2}\right) d u=\sum_{k=0}^{\infty} A_{k} \alpha_{2 k}\left(x^{\prime}\right) \alpha_{2 k}(x),
$$

where $A_{k}=1 /(2 k+1)$. Analogously,

$$
\begin{aligned}
r_{\text {III }}\left(x^{\prime}, x\right)= & \frac{1}{\pi^{2} U(0, x)} \int_{0}^{\infty} \exp \left(-u^{2}\right) f\left(x^{\prime}, u\right) f(x, u) d u= \\
& \frac{1}{U(0, x)} \sum_{k=0}^{\infty} A_{k} \alpha_{2 k}\left(x^{\prime}, \sigma\right) \alpha_{2 k}(x, \sigma),
\end{aligned}
$$

where

$$
f(x, u)=\operatorname{tg}^{-1} \frac{x+u}{\sigma}-\operatorname{tg}^{-1} \frac{x-u}{\sigma} .
$$

In these non-coherent cases the one-dimensional problem of the spectral line formation in a semi-infinite atmosphere the analog of Ambartsumian equation (2) has a form

$$
\begin{gathered}
\frac{2}{\lambda}\left[\alpha(x)+\alpha\left(x^{\prime}\right)\right] \rho\left(x^{\prime}, x\right)=r\left(x^{\prime}, x\right)+\int_{-\infty}^{\infty} \rho\left(x, x^{\prime \prime}\right) r\left(x^{\prime \prime}, x^{\prime}\right) \mathrm{d} x^{\prime}+ \\
\int_{-\infty}^{\infty} r\left(x, x^{\prime \prime}\right) \rho\left(x^{\prime \prime}, x^{\prime}\right) \mathrm{d} x^{\prime \prime}+\int_{-\infty}^{\infty} \rho\left(x, x^{\prime \prime}\right) \mathrm{d} x^{\prime \prime} \int_{-\infty}^{\infty} r\left(x^{\prime \prime}, x^{\prime \prime \prime}\right) \rho\left(x^{\prime \prime \prime}, x^{\prime}\right) \mathrm{d} x^{\prime \prime \prime},
\end{gathered}
$$

where $r\left(x^{\prime}, x\right)$ is either of redistribution functions. Bilinear expansions of $r\left(x^{\prime}, x\right)$ reduce the problem to solving the infinite set of functional equations for the functions $\varphi_{k}(x)$

$$
\varphi_{k}(x)=\alpha_{2 k}(x)+\frac{\lambda}{2} \sum_{m=0}^{\infty} A_{m} \int_{-\infty}^{\infty} \frac{\varphi_{m}(x) \varphi_{m}\left(x^{\prime}\right)}{\alpha(x)+\alpha\left(x^{\prime}\right)} \alpha_{2 k}\left(x^{\prime}\right) d x^{\prime},
$$

where $\alpha(x)$ is the profile of the absorption coefficient. This is the natural generalization of equation (4) to the case of partial redistribution over frequencies. Using expansions of types (27) and (30) makes it much easier to solve the 
corresponding transfer problems for the line-radiation (Yengibarian \& Nikoghossian, 1972a,b,c; 1973a,b; Yengibarian, Nikoghossian \& Gevorkian, 1975). Quasi-analytic and numerical methods have been developed for solving these problems (Nikoghossian \& Harutyunian 1976, 1978, 1979; Harutyunian \& Nikoghossian, 1978a,b; Nikoghossian, 1979, 1986a; Harutyunian, 1980a,b, 1985, 1991a,b; 1993, 1996). It was shown that the accuracy in solving the truncated set of equations (36) can be highly increased in view of the fact that to any fixed value of $x$ there corresponds the number $n$ beginning of which the functions $\varphi_{k}(x)$ may be replaced by the known functions $\alpha_{2 k}(x)$. Physically this reflects the fact that the formation of the far wings of the spectral line is due to the single scattering. Mathematically, this follows from the asymptotic behavior of functions $\alpha_{n}(x)$ in Eq.(28)

$$
\alpha_{n}(x) \approx 2^{(n+1) / 2} n^{n / 2} \exp \left[\left(x^{2}-n\right) / 2\right] \cos \left(\sqrt{2 n+1} x-\frac{n \pi}{2}\right),
$$

This allows reducing the solution of the infinite set of functional equations (36) to the set of equations of the form

$$
\varphi_{k}^{*}(x)=\alpha_{2 k}(x)+\frac{\lambda}{2} \sum_{m=0}^{N-1} A_{m} \int_{-\infty}^{\infty} \frac{\varphi_{m}^{*}(x) \varphi_{m}^{*}\left(x^{\prime}\right)}{\alpha(x)+\alpha\left(x^{\prime}\right)} \alpha_{2 k}\left(x^{\prime}\right) d x^{\prime}+\frac{\lambda}{2} \int_{-\infty}^{\infty} \frac{r\left(x^{\prime}, x\right) \alpha_{2 k}\left(x^{\prime}\right)}{\alpha(x)+\alpha\left(x^{\prime}\right)} d x^{\prime},
$$

and makes it possible to construct solutions of various problems of line-radiation transfer in the general case of frequency redistribution..

The works carried out in the 60s in this direction in the Byurakan observatory allowed not only to develop effective mathematical methods for solving problems of spectral line formation for partial redistribution of radiation in frequencies and directions, but also to compare the obtained numerical results with the data in the commonly used assumption of completely incoherent scattering. For instance, Harutynian \& Poghosyan (2018) numerically realized the algorithm for solving Ambattsumian's functional equations obtained for finite medium. Calculations were carried out for the particular case of the complete frequency redistribution. To this end, the authors elaborated the software package for calculating the nodes and corresponding weights of Hermite polynomials with high number of significant digits. The influence of the number of the used quadrature nodes, as well as the effect of the values of slab's thickness and the photon survival probability on the solution of problem were studied in details.

v. Statistical description of the radiation diffusion in an absorbing and scattering atmosphere. Ambartsumian's result Eq.(19) for determining the mean number of scattering events (MNSE) photons undergo during their diffusion in an absorbing and scattering atmosphere was treated and developed in a series of papers (Nikoghossian, 1984a,b, 1986b; Harutyunian \& Nikoghossian, 1983, 1987). A new approach was elaborated to determining any statistical average characterizing a diffusion process in an atmosphere. It is based on invariance principle and extensive use of generating functions. It was shown that the method is applicable to determining the statistical averages of continuously distributed random variables as well, though it is then necessary to use appropriate characteristic functions. As an illustration, the problem of the average time of a photon travel (ATT) in an atmosphere under assumption that it spends time only in travelling between scatterings was treated by Nikoghossian (1986b). In the general case, when the photons are thermalizing not only in scatterings but also in flight, this average makes it possible to gauge the relative importance of energy dissipation in the medium and of energy flow through a boundary. Another important application of this average is associated with the problems, frequently encountered in astrophysical applications, of the radiation of an atmosphere subject to non-stationary energy sources. In these problems, knowledge of the ATT makes it possible to ascertain whether radiative equilibrium in the medium is established.

Under general assumptions concerning the elementary scattering event, equations were obtained in Nikoghossian's papers for determining the MNSE and ATT in a plain-parallel semi-infinite atmosphere. It was shown that, for moving photons (i.e. not for those being trapped in the course of multiple scattering) the problem simplifies and is reduced to differentiation of the reflectance correspondingly over the parameter $\lambda$ for the MNSE and $\beta$ - for ATT. Here we limit ourselves by presenting few results obtained on the base of invariance principle.

It has been shown that the formula Eq. (19) is valid only for 'moving' photons, i.e. those that have not been truly absorbed in the atmosphere and thermalized. The required values of MNSE and ATT for the photons reflected from a semi-infinite atmosphere satisfy integral-differential equations easily obtained by the mentioned formal differentiation of the proper equation for the reflectance $\rho$. A separate approach has been developed to account for the number of scatterings of thermalized photons, which, in turn, makes it possible to find the desired average characteristics for all photons, regardless of their further 'fate'. For instance, the requisite values of $\operatorname{MNSE},\langle N(\tau, x, \eta)\rangle$ and $\operatorname{ATT}\langle\Omega(\tau, x, \eta)\rangle$ the photons with frequency-directional characteristics $(x, \eta)$ moving at optical depth $\tau$ undergo and spent during their traveling in the semi-infinite atmosphere, are found from equations

$$
\eta \frac{\partial\langle N(\tau, x, \eta)\rangle}{\partial \tau}=-v(x)\langle N(\tau, x, \eta)\rangle+\frac{\lambda}{2} \int_{-1}^{+1} d \eta^{\prime} \int_{-\infty}^{\infty} r\left(x, \eta ; x^{\prime}, \eta^{\prime}\right)\left\langle N\left(\tau, x, \eta^{\prime}\right)\right\rangle d x^{\prime}+v(x),
$$



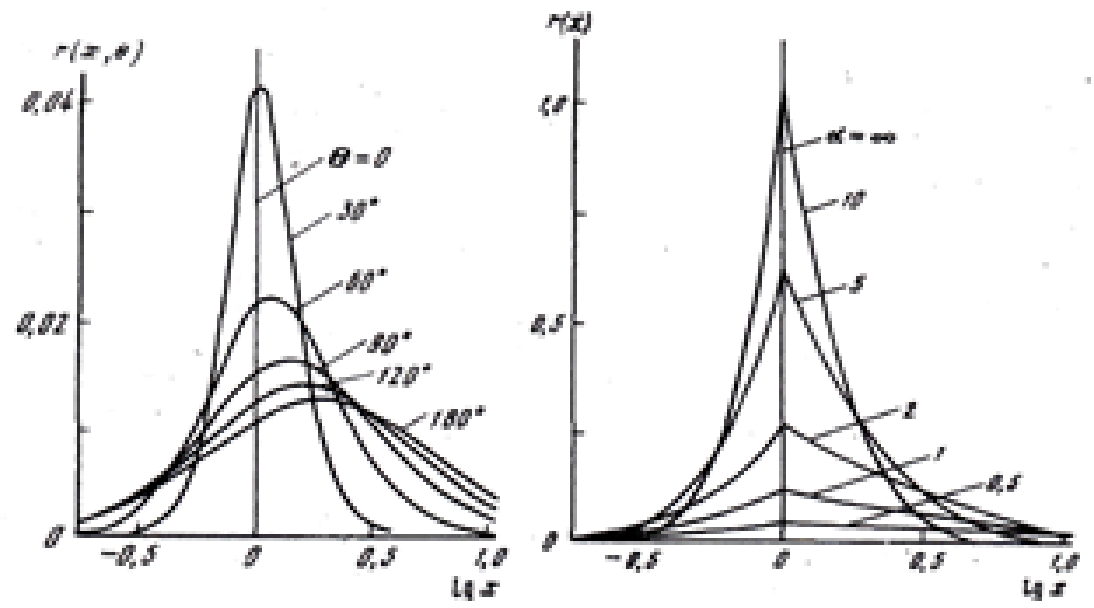

Figure 2: Frequency redistribution functions $r(x, \theta)$ and $r(x)$ for Compton scattering.

$$
\eta \frac{\partial\langle\Omega(\tau, x, \eta)\rangle}{\partial \tau}=-v(x)\langle\Omega(\tau, x, \eta)\rangle+\frac{\lambda}{2} \int_{-1}^{+1} d \eta^{\prime} \int_{-\infty}^{\infty} r\left(x, \eta ; x^{\prime}, \eta^{\prime}\right)\left\langle\Omega\left(\tau, x, \eta^{\prime}\right)\right\rangle d x^{\prime}+1,
$$

with the conditions $\langle N(0, x, \eta)\rangle=0,\langle\Omega(0, x, \eta)\rangle=0$ for $\eta>0$. Note that the time intervals represent the mean time taken in free path between two successive scattering events for a photon in the line center. The obtained equations differ from the transfer equations only by free terms, so that many of methods of solution for the latter cover these equations as well. It was proved that there is a rather general relation connecting considered average statistical values

$$
(1-\lambda)\langle N(\tau, x, \eta)\rangle+\lambda \beta\langle\Omega(\tau, x, \eta)\rangle=R_{0}(\tau, x, \eta),
$$

where $R_{0}(\tau, x, \eta)$ yields the probability the photon at optical depth $\tau$ with characteristics $(x, \eta)$ will be destroyed somewhere in the medium during its diffusion. Some of the presented results were generalized by Nikoghossian (2004b) to the case of inhomogeneous atmospheres.

vi. Compton scattering on free electrons. It is known that when interpreting the data obtained from observations of some astrophysical phenomena are widely used the mechanism of Compton scattering on electrons. In particular, this mechanism can be adopted to explain the sources of short X-ray bursts, X-ray radiation of quasars and nuclei of active galaxies (Zel'dovich \& Syunyaev, 1970; Illarionov \& Syunyaev, 1972; Basko, 1978). Before, the softening of the radiation in the course of multiple Compton scattering in the non-relativistic approximation was treated by Terebizh (1970).

It is of interest detection of the radiation spectra distortion caused by radiation scattering on free electrons and often referred to as "comptonization" of radiation. Especially .important in this case is the case of scattering on relativistic electrons, where the electrons transmit, in an average, some of the kinetic energy to the scattering photon. This phenomenon is commonly referred to as inverse Compton-effect.

To solve the problems of radiation transfer undergoing multiple Compton scattering, it is necessary to describe the elementary act of scattering, i.e., to know the law of the radiation redistribution on energy and directions. Under some simplifying assumptions the normalized redistribution function $r(x, \theta)$ analytically was derived by Harutyunian \& Nikoghossian (1980)

$$
r(x, \theta)=\frac{x}{2 K_{2}(\alpha) \sqrt{1+x^{2}-2 x \cos \theta}} \exp \left\{-\alpha \sqrt{\frac{1+x^{2}-2 x \cos \theta}{2 x(1-\cos \theta)}}\right\},
$$

where $\alpha=m_{e} c^{2} / k T_{e}, K_{2}(\alpha)$ is the cylindrical function of an imaginary argument of the second order, and $x$ is the ratio of frequency of the scattered photon to that of the incident photon. Hence the redistribution law averaged over all direction is of the form

$$
r(x)=\frac{|1-x|}{4 K_{2}(\alpha)} \int_{x_{0}}^{\infty} e^{-\alpha t}\left(t^{2}-1\right)^{-3 / 2} d t
$$

where $x_{0}=(1+x) / 2 \sqrt{x}$.

As it follows from Eq. (42), there is a drift in the frequency of the scattered photon towards high energy, the greater the scattering angle. These displacements are increasing also with decreasing $\alpha$. It is also seen that when scattered 
in the direction of motion of the original photon the energy of the latter does not change. The scattering indicatrix is lengthened in the opposite direction, the degree of which depends on the energy of electrons, Thus one can say that the most part of photons are scattered in the opposite direction with mostly increased energy. It is important to note that the redistribution function for the Compton scattering in this case depends only on the ratio of the scattered and incident photons energies. This implies that the global optical properties of the medium for this mechanism of scattering are also the functions of the mentioned ratio.

The problem of diffuse reflection from a semi-infinite medium with Compton scattering on free electrons was considered by Harutyunian \& Nikoghossian (1981). The rigorous statement of the problem in terms of the invariance procedure was given.

The elementary events of photon scattering by a chaotically moving monoenergetic electron gas has been examined by Harutyunian (2006) to obtain the angle-averaged redistribution function. Expressions were obtained for the averaged redistribution function and its asymptotes which describe the redistribution function for low frequency radiation. The question of determining the frequency-dependent absorption coefficient was discussed, as well.

vii. Intensity fluctuations. Dynamically active media. The need to create a theory of radiative transfer in media with a complicated fine structure subject to random variations has long been felt. Already in the 1970's (Cram, 1972) it was shown that the observed profiles of chromospheric lines of ionized calcium cannot be explained in terms of the theory developed for homogeneous stationary media. It is quite evident that a satisfactory interpretation of other nonstationary phenomena in the solar atmosphere, such as chromospheric spicules, coronal streams, and polar plums would require a new theory. Problems involving the propagation of radiation in randomly inhomogeneous media also appear in other branches of astrophysics. Examples include the scattering of light on molecular clouds in the interstellar medium and the stochastic attenuation of galactic radiation as it travels over cosmologic distances.

The first papers in the area appeared in the frameworks of scientific collaboration between Meudon Observatory in France and Byurakan Observatory examined the line formation problems in multicomponent static media, i.e. the possible motions of structural elements were ignored (Nikoghossian, Pojoga \& Mouradian, 1997, 1998a,b; Nikoghossian \& Mouradian, 2000; Nikoghossian, 2002; Nikoghossian, Aboudarham \& Mouradian, 2005). The direct implication of this research was interpretation of the EUV spectra of solar quiescent prominences (see below, Sect. 7, (i)).

An analytical procedure explored by Nikoghossian (2002) aimed at constructing the profiles of the spectral lines formed in a stochastic multicomponent atmosphere. A model one-dimensional problem was solved for the atmospheres in both local thermodynamic equilibrium (LTE) and non-LTE. The results previously obtained for the LTE atmosphere were generalized to include, in particular, correlations of the random variations in the physical properties of the structural elements. The line profiles formed in LTE stochastic atmosphere may have rather complicated shapes and, in general, differ significantly from those specific to the averaged homogeneous atmosphere. Particularly, it was shown that the stochastic multicomponent atmosphere may produce a kind of double-peaked profile, which is usually associated with the multiple scattering effects. The influence of the Markovian correlations between structural elements on the line profile was revealed.

The problem of the line formation in a stochastic non-LTE atmosphere was considered under assumption of the complete redistribution over frequencies with allowance for the absorption in continuum. For the line profile, a closed-form analytical expression was derived. The computed profiles were compared with those for a homogeneous deterministic atmosphere with preliminarily averaged physical properties. This allows one to conclude that the discrepancy between the line profiles relevant to these two cases may become significant, whereas the normalized profiles are fairly close to each other. The evident advantage of the model problem discussed in the cited papers is that it provides an important insight into specificity of the line radiation in an inhomogeneous atmosphere with randomly varied properties and it allows for various generalizations.

The theory was developed in (Nikoghossian, 2002, 2005) to determine the mean intensity and the relative mean square deviation (RelMSD) for the line radiation emerging from an one-dimensional atmosphere with randomly distributed inhomogeneities. Both the local thermodynamic equilibrium (LTE) and Non-LTE cases were considered. The results previously obtained for the LTE atmosphere were extended to encompass the more realistic situation when the random parameters describing the physical properties of structural elements may take an arbitrary number of possible values. The profiles of spectral lines constructed by solving the stochastic problem of radiative transfer in the LTE atmosphere allow one to check the accuracy of the commonly used approximations.

The LTE problem treating the number $N$ of structural elements each of which is described by the optical thickness $\tau$, and the power of the energy release, $B$ (the value of $B$ is assumed to be constant within each individual element) was presented by (Nikoghossian, Pojoga \& Mouradian, 1999). The paper considers the simplest situation, when the pair of quantities $(B, \tau)$ takes randomly only two possible sets of values with some probabilities $p_{1}$ and $p_{2}$. For the averaged characteristics of the emerging radiation such as the mean intensity $\left\langle I_{N}\right\rangle$ and the RelMSD $\delta_{N}$ were found 


$$
\left\langle I_{N}\right\rangle=L_{N}\left\langle I_{1}\right\rangle, \quad \delta_{N}=\frac{M_{N}}{L_{N}^{2}}\left(1+\delta_{1}\right)+2 \frac{K A_{N}}{\left\langle I_{1}\right\rangle L_{N}^{2}}-1,
$$

where the following notations are used

$$
L_{N}=\left(1-\alpha^{N}\right) /(1-\alpha), \quad M_{N}=\left(1-\beta^{N}\right) /(1-\beta), \quad A_{N}=\left(L_{N}-M_{N}\right) /(\alpha-\beta),
$$

and $\alpha=\langle\exp (-\tau)\rangle, \beta=\langle\exp (-2 \tau)\rangle, K=\langle J \exp (-\tau)\rangle, J=\langle B(1-\exp (-\tau))\rangle$.

An important salient trait inherent in Eqs. (43) is that the values of the mean intensity and the RelMSD for the multicomponent atmosphere with an arbitrary number of elements are determined by only a few parameters. These parameters for the general case of $n$ realizations of $(B, \tau)$ are $\alpha, \beta,\left\langle I_{1}\right\rangle=\sum_{i=1}^{n} p_{i} J_{i} K=\sum_{i=1}^{n} p_{i} J_{i}\left(1-\exp \left(-\tau_{i}\right)\right)$, and

$$
\delta_{1}=\frac{1}{2\left\langle I_{1}\right\rangle^{2}} \sum_{i=1}^{n} p_{i} \sum_{k=1}^{n} p_{k}\left(J_{i}-J_{k}\right)^{2} .
$$

All these five parameters are the average characteristics of a single structural element so that, in general, there must exist a great number of configurations, having a fixed number of components, that are characterized by the same values of the mean intensity and the RelMSD and, moreover, by the same behavior with varying $N$. The problem of determining the statistical characteristics of the line radiation formed in a multicomponent Non-LTE atmosphere was first treated for the case of conservative scattering. It was derived a closed-form analytical expression for the RelMSD of the outgoing intensity for an arbitrarily large number of structural elements in the medium.

The theoretical results were employed for interpreting some of the features specific to the spatial brightness fluctuations of prominences in extreme ultraviolet (EUV) lines (see Sect. 7.i). The estimates obtained for the mean value of the line-of-sight number of threads are in agreement with those inferred by the other authors. Some important conclusions on the multithread structure of prominences were drawn.

The theory was generalized afterwards to cover the frequency-dependent problem in (Nikoghossian, 2002; Nikoghossian, Aboudarham, Mouradian, 2007). It was particularly concluded that when the intensity fluctuations of radiation escaping from a multicomponent atmosphere are due to random variations in the distribution of internal energy sources, the RelMSD of the measured intensity decreases monotonically from the center of the line to its wings, while if these fluctuations are resulted mostly from the difference in opacities of structural elements the run is opposite: the RelMSD grows towards wings.

Further generalization of the theory concerns the dynamically active stochastic and multicomponent atmospheres (Nikoghossian, 2007a). Two types of problem for the LTE lines were discussed. In the first it was assumed that the realization of one or another type of non-thermal motion depends on the type of structural element and in the other this type of dependence is absent, i.e., it was supposed that the assumed random values of the velocity are distributed according to a law that is common to all the components.

The general effect of large-scale motions on spectral line profiles and on the scale of their spatial (or temporal) fluctuations was considered for spectral lines in LTE, which can be regarded, with a high probability, as valid for most of the non-hydrogen lines in the EUV spectrum of quiescent prominences. The theoretical results were compared with observational data obtained with the SUMER spectrometer in the SOHO space mission (Fig.3). The distinctive feature of the RelMSD of the radiation in a line formed in a dynamically active stochastic atmosphere is local "spikes" (maxima) in the wings of the line. The theoretical results obtained were compared with spectral observations of quiescent solar prominences obtained in framework of the SOHO space mission.

The "spikes" (local maxima) in the wings of the lines predicted by the above theory for non-thermal (hydrodynamic) motions can be seen distinctly. It can also be seen that for lines with a high effective formation temperature (i.e., the lines formed primarily in the surrounding corona), such as OVI $\lambda \lambda 1032 \AA, \lambda \lambda 1037 \AA$, and NV $\lambda \lambda 1242 \AA$, the magnitude of these spikes appears to be smaller than for the lines formed entirely within the prominence. The appearance of isolated single spikes is an indication that the velocities of the non-thermal motions are only slightly greater than the thermal speed, if they exceed it at all.

viii. Turbulent atmospheres. The formation of spectral lines in a turbulent atmosphere with a spatially correlated velocity field were examined in a series of papers Nikoghossian (2007b,c, 2017). A new approach for solving this problem proposed in the first of these papers does not base on the Fokker-Planck formalism commonly used in the literature. The invariant imbedding method makes it possible to reduce the problem of finding the mean radiant intensity observed in a line to solving a system of differential equations. This possibility is based on determining the mean intensity of the radiation emerging from the medium for a fixed value of the turbulent velocity at its boundary (Yengibarian \& Nikoghossian, 1969). A separate integral equation is derived for this quantity. The dependence of the line profile, integrated intensity, and width on the mean correlation length and the average value of the hydrodynamic velocity was studied. It was shown that the transition from a micro-turbulent regime to a macro-turbulent regime occurs within a comparatively narrow range of variation in the correlation length. Fig.4 demonstrates the dependence 


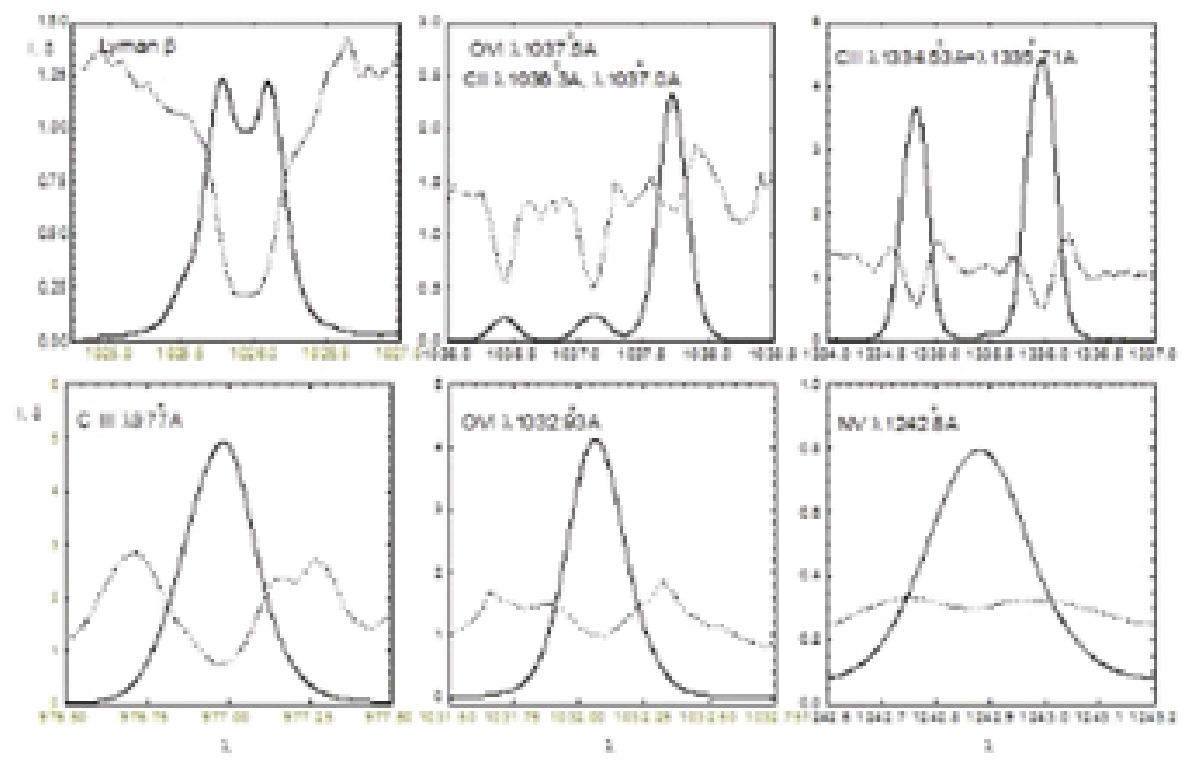

Figure 3: Statistical mean profiles (smooth curves) and the corresponding relative mean square deviations (dotted curves) for a series of relatively strong EUV lines of a solar prominence observed on August 10, 1999, as part of the SOHO space mission.

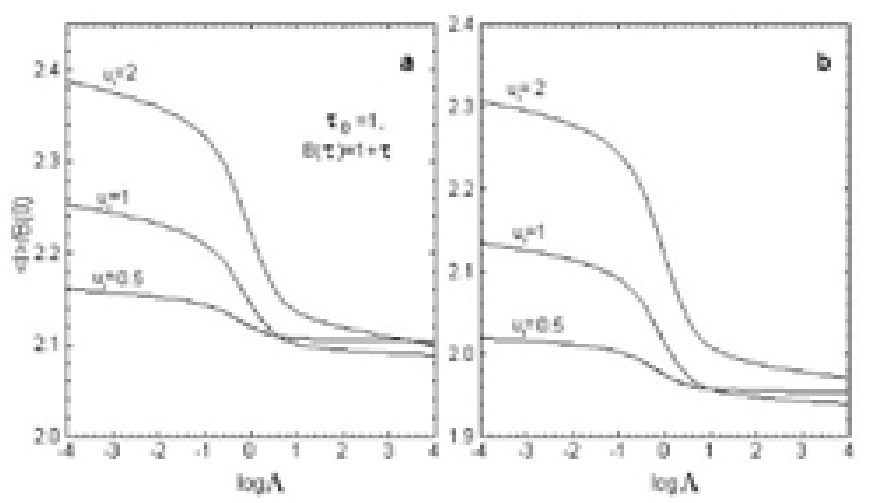

Figure 4: Integrated intensities of spectral lines observed at the boundaries $\tau=\tau_{0}$ (a), and $\tau=0$ (b) of a turbulent atmosphere of optical thickness $\tau_{0}=1$, with the energy sources $B(\tau)$ that vary linearly with depth.

of intensities emergent from an atmosphere of optical thickness $\tau_{0}=1$ on the correlation length $\Lambda$. The sources of energy in the medium are assumed to be linearly varying with optical depth.

The proposed method yields a solution to the problem for a family of inhomogeneous atmospheres with different optical thicknesses, which makes it easy to determine the radiation field inside the turbulent medium. This approach can be generalized in various ways, in particular, it can be applied without significant changes to the case, where the correlation length depends on the position within the atmosphere.

The diffuse reflection of line radiation from a one dimensional semi-infinite turbulent atmosphere was examined in (Nikoghossian, 2007c) in two limiting regimes of micro- and macro-turbulence assuming that turbulence developed in the atmosphere is homogeneous. This means that the hydrodynamic velocity vector $\mathbf{v}$ is a random function that depends on depth, while the average characteristics of the velocity field are independent of depth in the atmosphere (Batchelor, 1970). Because of the homogeneity of the process, the correlation coefficient $l=\left|\tau-\tau^{\prime}\right|\left(\tau, \tau^{\prime}\right.$ - optical depths) depends only on the distance $\rho$ between two points. It was also assumed that the probability law according to which the hydrodynamic velocity $u$, measured in units of the mean thermal speed, takes one or another value is also independent of depth. In addition, it was supposed that the variations in the velocity inside the medium are correlated with one another. It was found the mean intensity of the radiation emerging from the boundary of the medium. The effect of the radiation on the velocity field was neglected. Formulated in this way, the problem models the case, often realized in astrophysics, where the optical depth of a radiating medium is large in the absence of turbulence. 


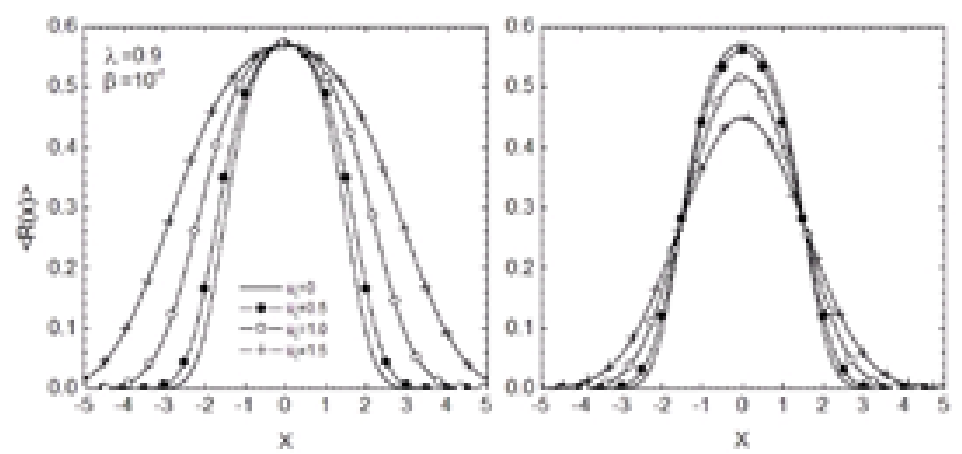

Figure 5: Profiles of lines formed during reflection from a semi-infinite micro-turbulent (left) and macro-turbulent (right) atmospheres. The central intensity of a line formed by reflection from a micro-turbulent atmosphere essentially remains unchanged as the average non-thermal velocity increases. In the macro-turbulent regime, the equivalent width of the line does not change.
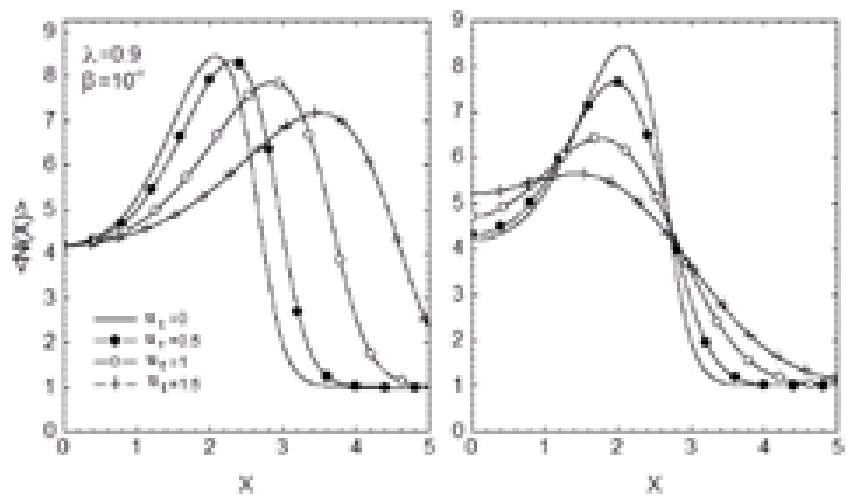

Figure 6: Mean number of scattering events experienced by a photon during diffusion in an atmosphere with micro(left) and macro-turbulence (right). True absorption in the continuum is also treated as scattering.

The problem was solved by applying invariance principle. In addition to the observed spectral line profile, statistical averages describing the diffusion process in the atmosphere (mean number of scattering events, average time spent by a photon in the medium) were determined. The dependence of these quantities on the average hydrodynamic velocity and scattering coefficient was shown in Figs.4-7. It was shown that in the micro-turbulent regime the intensity at the line center depends only slightly on the mean non-thermal velocity. In both regimes, photons in the far wings undergo scattering more frequently than in a static atmosphere, although they spend, on average, less time in it.

The method of invariant imbedding used in the above-mentioned papers enables solution of the problem under rather general assumptions about the character of the turbulence, as well as about elementary scattering events and the distribution of energy sources in the medium. It was concluded that in the case of micro-turbulence, the reflectivity of the medium and its opacity are low over the entire frequency range. It was also found that the dependence of the observed characteristics on the correlation length is stronger when the medium is thicker and the average velocity of the turbulent motions is higher.

ix. Non-linear relations of the radiative transfer theory. As early as in 1977 Rybicky derived quadratic integrals of the transfer equation, however, some important points risen there remained unanswered for a long time. He studied the problems, for which the transfer equation admits integrals that involve quadratic moments of the radiation field. When the boundary conditions (or other constraints) are required to be met, the integrals convert into nonlinear relations of specific form for quantities characterizing the radiation field in the atmosphere. The so-called $Q$ - and $R$ - integrals involve as a particular case a certain class of 'surface' results, some of which were known well in the transfer theory.

Further generalization of Rybicki's results for monochromatic, isotropic scattering in the plane-parallel medium was given in (Ivanov, 1978). The so-called 'two-point' relations have been found that couple the intensities of equally directed radiation at two different depths in the atmosphere. The more general concept of 'bilinear integrals' (or relations) was introduced in (Nikoghossian, 1997) for quadratic integrals that connect the radiation fields of two separate transfer problems but referred to the same optical depth. Following the given terminology, it was reasonable to introduce also the concept of 'two-point bilinear integrals' for those coupling with each other the radiation fields 


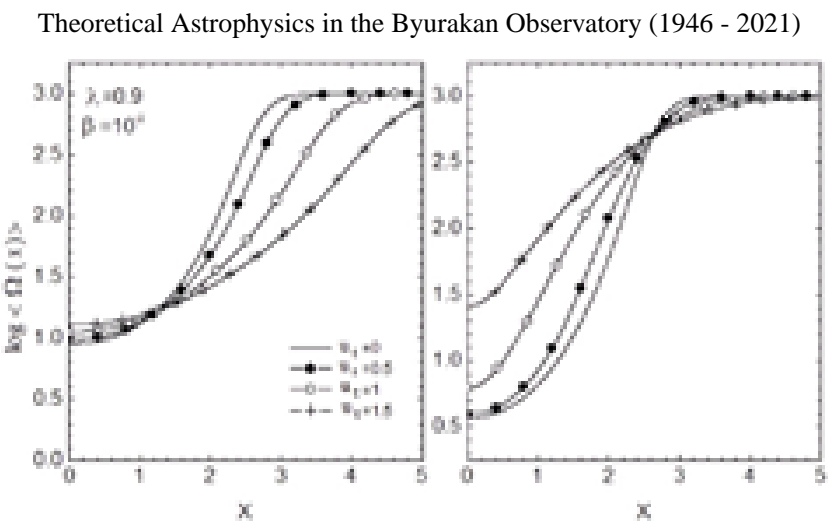

Figure 7: Average photon residence time in semi-infinite micro- (left) and macro-turbulent (right) atmospheres.

referred to both different transfer problems and diverse optical depths. Evidently this latter type of relations comprises all other types as the specific cases. Regardless of the new results, the main question on the physical nature of existence of quadratic and bilinear relations remained abstruse for a long time until appearing the mentioned Nikoghossian's paper. The idea that there must exist some relationship to the invariance principle suggested itself and is justified if for no other reason than that the majority of nonlinear equations in the transfer theory are associated in some or other way with the invariance technique. Most commonly these equations admit a plain physical interpretation and can be established by means of a direct use of the mathematical model of the physical process. Having this in mind, one may naturally be tempted to derive the requisite bilinear relations by the similar manner, thus making clear the physical significance of these relations. This kind of attempt has been made by Hubeny (1987a,b), who provided some intuitive insight into physical nature of quadratic results of the transfer theory.

The more general and mathematically rigorous derivation of quadratic and bilinear relations was given in (Krikorian \& Nikoghossian, 1996, Nikoghossian, 1999) on the base of the principle of invariance. The authors elucidated the profound connection between invariance principle, quadratic relations and conservation laws resulting from general variational principle.

Due to the importance of the results obtained, it is expedient to reproduce here some of them for the simplest case of monochromatic, isotropic scattering in a semi-infinite, plane-parallel atmosphere. Suppose also that the atmosphere is homogeneous and does not contain energy sources. Turning to Eqs. (2), (3) and making use the expression for the zero-moment $\alpha_{0}$ of $\varphi(\eta)$

$$
\alpha_{0}=\int_{0}^{1} \varphi(\eta) d \eta=2(1-\sqrt{1-\lambda}) / \lambda
$$

one can derive

$$
(1-\lambda)(\eta+\xi) \rho(\eta, \xi)=\frac{\lambda}{2}\left(1-\int_{0}^{1} \rho\left(\eta, \eta^{\prime}\right) \eta^{\prime} d \eta^{\prime}\right)\left(1-\int_{0}^{1} \rho\left(\xi, \eta^{\prime}\right) \eta^{\prime} d \eta^{\prime}\right) .
$$

Alongside with the reflection coefficient, it is of importance the function $Y(\tau, \eta, \mu)$ that characterizes the probability of the photon exit from atmosphere in the direction $\mu$, if originally it was moving at depth $\tau$ with the directional cosine $\eta$. The symmetry property of the $Y$ - function follows from the reciprocity principle and can be represented in the form:

$$
|\eta| Y(\tau, \eta, \mu)=|\mu| Y(\tau,-\mu,-\eta)=|\mu| \tilde{Y}(\tau, \mu, \eta),
$$

where for convenience it was introduced the function $\tilde{Y}$ with angular arguments referenced from inner normal direction. This function also admits a probabilistic interpretation, namely, $\tilde{Y}(\tau, \eta, \mu) d \eta$ is the probability that a photon incident on the atmosphere with the directional cosine $\mu$ will move (in general, as a result of multiple scattering) at depth $\tau$ within the directional interval $(\eta, \eta+d \eta)$. It is clear that $\tilde{Y}(0, \mu, \eta)=\eta \rho(\eta, \mu)$. In fact, $\tilde{Y}(\tau, \mu, \eta) /|\eta|$ is none other than the Green function for the source-free problem called also the surface Green function (Case \& Zweifel, 1967). This quantity completely determines the radiation field throughout the semi-infinite atmosphere that is illuminated by the external mono-directional source of unit intensity. Taking into account that

$$
Y(\tau,-\eta, \mu)=\int_{0}^{1} Y\left(\tau, \eta^{\prime}, \mu\right) \rho\left(\eta^{\prime}, \eta\right) \eta^{\prime} d \eta^{\prime}, \quad \tilde{Y}(\tau, \mu,-\eta)=\eta \int_{0}^{1} \tilde{Y}\left(\tau, \mu, \eta^{\prime}\right) \rho\left(\eta, \eta^{\prime}\right) d \eta^{\prime} .
$$

and using Eq. (47), it is easy to obtain

$$
\int_{-1}^{1} Y(\tau, \xi, \mu) Y\left(\tau^{\prime},-\xi, \mu^{\prime}\right) d \xi=\frac{\lambda}{2}\left(\int_{-1}^{1} Y(\tau, \xi, \mu) d \xi\right)\left(\int_{-1}^{1} Y\left(\tau^{\prime}, \xi, \mu^{\prime}\right) d \xi\right)
$$


We shall see below that the described procedure admits a simple physical interpretation which makes it possible to obtain the same result directly by means of some modification of invariance idea. The probabilistic meaning assigned to the function $Y$ sets one thinking that some statistical explanation may be suggested for equation (50) as well. Indeed, this equation implies that $\lambda / 2$ can be regarded as the correlation coefficient of two random events so that this result can be stated in the probabilistic language as follows.

Two random events of two photons exit from a semi-infinite atmosphere in certain fixed (diverse, in general) directions, if they were originally moving in opposite directions at some different optical depths, are correlated with the correlation coefficient equaled to $\lambda / 2$.

The second fundamental result generating quadratic and bilinear $R$-relations, can be found by a similar manner from Eq. (47). Multiplication of this equation by $\tilde{Y}(\tau, \mu, \eta) \tilde{Y}\left(\tau^{\prime}, \mu^{\prime}, \xi\right)$, and integration over $\eta$ and $\xi$ in the range $(0,1)$, in light of the second of Eqs. (49), yields

$$
(1-\lambda) \int_{-1}^{+1} \tilde{Y}(\tau, \mu, \xi) \tilde{Y}\left(\tau^{\prime}, \mu^{\prime},-\xi\right) d \xi=\frac{\lambda}{2}\left(\int_{-1}^{+1} \tilde{Y}(\tau, \mu, \xi) \xi \frac{d \xi}{|\xi|}\right)\left(\int_{-1}^{+1} \tilde{Y}\left(\tau^{\prime}, \mu^{\prime}, \xi\right) \xi \frac{d \xi}{|\xi|}\right) .
$$

With use of the reversibility property Eq. (48) in Eq. (51), one can obtain

$$
(1-\lambda) \int_{-1}^{+1} Y(\tau, \xi, \mu) Y\left(\tau^{\prime},-\xi, \mu^{\prime},\right) \xi^{2} d \xi=\frac{\lambda}{2}\left(\int_{-1}^{1} Y(\tau, \xi, \mu) \xi d \xi\right)\left(\int_{-1}^{1} Y\left(\tau^{\prime}, \xi, \mu^{\prime}\right) \xi d \xi\right) .
$$

Relations (51), (52) constitute a wealth of information about radiation fields in the source-free media. Some of their versatile consequences will be examined below. Here merely the special results following from these relations, when $\tau=\tau^{\prime}$ and $\mu=\mu^{\prime}$ are presented

$$
\begin{gathered}
\int_{0}^{1} Y(\tau, \xi, \mu) Y(\tau,-\xi, \mu) d \xi=\frac{\lambda}{4}\left(\int_{-1}^{+1} Y(\tau, \xi, \mu) d \xi\right)^{2} \\
(1-\lambda) \int_{0}^{+1} Y(\tau, \xi, \mu) Y(\tau,-\xi, \mu,) \xi^{2} d \xi=\frac{\lambda}{4}\left(\int_{-1}^{+1} Y(\tau, \xi, \mu) \xi d \xi\right)^{2} .
\end{gathered}
$$

Thus, we arrived at quadratic $Q$ - and $R$ - relations representing the prototypes of those obtained in (Rybicky, 1977). The derived bilinear and quadratic relations may be applied to different transfer problems of astrophysical interest to give a number of new results.

Let us start, for instance, with the problem of diffuse reflection from a semi-infinite atmosphere, which is illuminated from outside by parallel beam of radiation of unit intensity with directional cosine $\mu$. Using superscripts ' + ' and '-' to denote the intensities with angular arguments $+\eta$ and $-\eta$, respectively, by virtue of the probabilistic meaning of function $Y$ given above, one may write

$$
I^{+}(\tau, \eta, \mu)=\tilde{Y}(\tau, \mu,-\eta) / \eta, \quad I^{-}(\tau, \eta, \mu)=\tilde{Y}(\tau, \mu, \eta) / \eta
$$

Now equations Eqs. (50) and (51) correspondingly yield

$$
\begin{gathered}
Q\left(\tau, \mu ; \tau^{\prime} \mu^{\prime}\right)=\lambda J(\tau, \mu) J\left(\tau^{\prime}, \mu^{\prime}\right), \\
(1-\lambda) R\left(\tau, \mu ; \tau^{\prime} \mu^{\prime}\right)=\lambda H(\tau, \mu) H\left(\tau^{\prime}, \mu^{\prime}\right),
\end{gathered}
$$

where

$$
\begin{aligned}
& J(\tau, \mu)=\frac{1}{2} \int_{-1}^{+1}\left[I^{+}(\tau, \eta, \mu)+I^{-}(\tau, \eta, \mu)\right] d \eta \\
& H(\tau, \mu)=\frac{1}{2} \int_{-1}^{+1}\left[I^{+}(\tau, \eta, \mu)-I^{-}(\tau, \eta, \mu)\right] \eta d \eta
\end{aligned}
$$

are the mean intensity and flux, respectively; we introduced also two quadratic moments of the radiation field given by

$$
\begin{gathered}
Q\left(\tau, \mu ; \tau^{\prime} \mu^{\prime}\right)=\frac{1}{2} \int_{-1}^{+1}\left[I^{+}(\tau, \eta, \mu) I^{-}\left(\tau^{\prime}, \eta, \mu^{\prime}\right)+I^{+}\left(\tau^{\prime}, \eta, \mu^{\prime}\right) I^{-}(\tau, \eta, \mu)\right] d \eta \\
R\left(\tau, \mu ; \tau^{\prime} \mu^{\prime}\right)=\frac{1}{2} \int_{-1}^{+1}\left[I^{+}(\tau, \eta, \mu) I^{-}\left(\tau^{\prime}, \eta, \mu^{\prime}\right)+I^{+}\left(\tau^{\prime}, \eta, \mu^{\prime}\right) I^{-}(\tau, \eta, \mu)\right] \eta^{2} d \eta
\end{gathered}
$$


The angular arguments $\mu$ and $\mu^{\prime}$, which specify the directions of incidence, enter into Eqs.(??) as a parameter so that the relations of this type can be written for arbitrary angular distribution of illuminating radiation. Moreover, they enable one to establish the relationship between radiation fields of two diverse problems with different angular distribution for incident radiation.

An important insight into the source-free problem and the group of related problems can be given upon the simple and physically intelligible formula, second of Eq. (49) which was employed in deriving Eqs.(??). By virtue of formulas Eq. (55) it can be rewritten as

$$
I^{+}(\tau, \eta, \mu)=\eta \int_{0}^{1} \rho(\eta, \varsigma) I^{-}(\tau, \varsigma, \mu) d \varsigma
$$

which states the obvious fact that, in the absence of internal sources, the upward radiation (and ultimately the radiation field at any optical depth $\tau$ is completely determined by the intensity of inward radiation. Indeed, taking account of that

$$
I^{-}(\tau, \eta, \mu)=\lambda \int_{0}^{\tau} J(\tau, \mu) e^{-\frac{\varsigma-t}{\eta}} \frac{d t}{\eta}+\frac{1}{2} \delta(\eta-\mu) e^{-\frac{\tau}{\mu}}
$$

and the source function $S(\tau, \mu)=\lambda J(\tau, \mu)$. The formula (61) being inserted into Eq. (60) yields

$$
S(\tau, \mu)=\int_{0}^{\tau} L(\tau-t) S(t, \mu) d t+\frac{\lambda}{2} \varphi(\mu) e^{-\frac{\tau}{\mu}} .
$$

Thus, knowledge of the reflectance of the atmosphere makes it possible to reduce the classical boundary-value problem of determining the internal field of radiation to the Volterra-type associated with an initial value problem. This result is not unexpected, however, and is important in the sense that similar equations may be written (see below) for some special internal-source problems as well while formula (60) is no more valid.

It has been shown that discussion similar to that given above be carried out for some other classic problems of the theory of radiative transfer. For instance, consider the atmosphere with the uniformly distributed internal sources of energy so that

$$
S(\tau)=\lambda J(\tau)+(1-\lambda) B
$$

where $B=$ const is related to the Planck function. Two-point quadratic relations now have a form

$$
\begin{gathered}
Q\left(\tau, B ; \tau^{\prime}, B^{\prime}\right)=\lambda S(\tau, B) S\left(\tau^{\prime}, B^{\prime}\right)-(1-\lambda) B B^{\prime}, \\
(1-\lambda) R\left(\tau, B ; \tau^{\prime}, B^{\prime}\right)=\lambda H(\tau, B) H\left(\tau^{\prime}, B^{\prime}\right)+ \\
(1-\lambda)\left[B K\left(\tau^{\prime}, B^{\prime}\right)+B^{\prime} K(\tau, B)\right]-(1-\lambda) B B^{\prime} / 3,
\end{gathered}
$$

where

$$
K(\tau, B)=\frac{1}{2} \int_{0}^{1}\left[I^{+}(\tau, \eta, B)+I^{-}(\tau, \eta, B)\right] \eta^{2} d \eta
$$

is the $K$-moment and, for clarity, we marked $B$ explicitly as an argument identifying the problem under consideration. It was shown that here again the source satisfies Volterra-type integral equation analogous to that of Eq. (62)

$$
S(\tau, B)=\int_{0}^{\tau} L(\tau-t) S(t, B) d t+B \sqrt{1-\lambda} .
$$

Using the same approach, two-point quadratic relations were derived also for the Milne problem and for the radiation transfer problem concerning the atmosphere with the exponential distribution of internal energy sources of the form $b(\tau, m)=(1-\lambda) B e^{-m \tau}$ with constant $B$ and $m$. Sending the reader after $Q$ - and $R$ - non-linear relations to [17], we limit ourselves by presenting here the equations, counterparts of Eqs. (62), (67) for corresponding source functions

$$
S(\tau, F)=\int_{0}^{\tau} L(\tau-t) S(t, F) d t+(\sqrt{3} / 4) F
$$

where $F$ is the constant factor in the Milne problem known as astrophysical flux, and

$$
S(\tau, m)=\int_{0}^{\tau} L(\tau-t) S(t, m) d t+(1-\lambda) B \varphi\left(m^{-1}\right) e^{-m \tau} .
$$

It must be emphasized that all the presented relations are valid if only $\lambda=$ const, more generally, they are true wherever the local optical characteristics of the atmosphere do not change. 


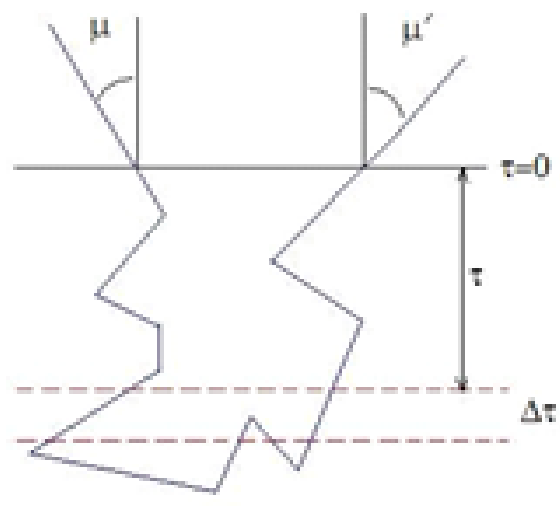

Figure 8: Derivation of the two-point bilinear $Q$ relation.

x. The modified principle of invariance. The preceding results suggest an idea on that there must exist a close inter-connection between bilinear relations and the invariance principle. For this reason, let us return to the original formulation of the invariance principle given in the outset of the review. Consider a semi-infinite, plane-parallel and source-free homogeneous atmosphere. For simplicity of exposition, the probabilistic approach to the problem will be adopted.

Let a photon is incident upon the boundary plane $\tau=0$ of the atmosphere at an angle $\cos ^{-1} \mu$ (Fig.8) to the inner normal, and we are interested in the probability of the photon exit at some angle $\cos ^{-1} \mu \prime$ to the upper normal direction. The classical formulation of the invariance principle assumes the addition (or removal) of a thin layer to (from) the surface of the atmosphere, presuming the optical properties of the layer and atmosphere to be the same. It is completely clear, however, that physically there is no difference whether we add (or remove) the layer to (from) the top of the atmosphere or do it somewhere within it with some selected layer $(\tau, \tau+\Delta)$. In the latter case we are interested in the probability of reflection subject to condition that the level $\tau$ intersected. Recalling the probabilistic meaning of functions $Y$ and $\tilde{Y}$ introduced in the outset of this subsection, it is readily seen that the probability of the mentioned event is

$$
d \mu \int_{0}^{1} Y\left(\tau,-\varsigma, \mu^{\prime}\right) \tilde{Y}(\tau, \mu, \varsigma) d \varsigma
$$

Further, we must determine the same probability accounting of the elementary processes of interaction with the selected layer $(\tau, \tau+\Delta)$ and equate to that of the formula Eq. (70) in accordance with what have been said. One may distinguish three types of processes of the first order of $\Delta \tau$ associated with the layer.

a.The scattering photon passes the selected layer with no interaction.

b.The photon enters the selected layer by crossing the plane $\tau$ and is scattered in the layer in some direction specified by $\eta$.

c.The photon enters the selected layer from below, i.e. by crossing the plane $(\tau+\Delta \tau)$ and is scattered in the layer. The proper probabilities for these three events are derived in (Nikoghossian, 1997). By adding up the obtained values, after some simple transformation we arrive at the specific version of the bilinear $Q$ - relation (50) written for $\tau=\tau^{\prime}$.

$$
\int_{-1}^{1} Y(\tau, \xi, \mu) Y\left(\tau,-\xi, \mu^{\prime}\right) d \xi=\frac{\lambda}{2}\left(\int_{-1}^{1} Y(\tau, \xi, \mu) d \xi\right)\left(\int_{-1}^{1} Y\left(\tau, \xi, \mu^{\prime}\right) d \xi\right)
$$

Finally, on setting $\tau=0$ in and taking account of the boundary condition $Y(0, \zeta, \mu)=\delta(\zeta-\mu)$ we are led to invariance equation (2) for the reflection coefficient. We see that equation (71) is more informative (as compared to Eq. (??) and can be regarded as the extension of Ambartsumian's equation to all depths in the atmosphere.

xi. The variational formalism. As was above said, the theory of radiative transfer can be made to rest on the principles of invariance. It was shown that the invariance equation, being combined with some simple physical reasoning, makes it possible to derive the more informative quadratic and bilinear relations, for which the former equation is a special (surface) result. It turned out that the resulting $Q$ - relations may be envisioned as a manifestation of a somewhat generalized version of the classical principle of invariance and can be obtained immediately. These facts indicate the fundamental nature of the invariance property of transfer problems and set one thinking that there must exists some general formulation of problems that implies both the equation of transfer and the principles of invariance as a kind of laws.

As it was shown by Nikoghossian (1999), the problems of transfer of radiation in the plane-parallel homogeneous atmosphere admit a variational formulation, the equation of transfer then being the Euler - Lagrange equation and the 
bilinear $Q$ - relation being the conservation law due to form-invariance of the suitable Lagrangian. In fact, a single functional comprises all the information on features of the problem and allows a systematic connection between symmetries and conservation laws. Being the first integrals of the Euler - Lagrange equation, the conservation laws may facilitate the solution of the problem under consideration and assist in its interpretation. Two salient problems, encountered in having recourse to the variational principle, are the existence of the principle for a given problem and the derivation of appropriate conservation laws. The former of these problems for systems of partial differential equations was solved by Vainberg (1964), who showed that this problem is equivalent to determining whether an operator is potential or not. The derivation of the conservation laws is based on Noether's theorem (see, Gelfand \& Fomin, 1965), which suggests a systematic procedure for establishing these laws from a direct study of the variational integral. An important generalization of the Noether's theorem to encompass the integral-differential equations was given by Tavel (1971).

While the variational approach is widely used in various branches of theoretical physics, it was not the case in the field of the radiative transfer theory, with the only exception being the paper of Anderson (1973) who employs Tavel's results to establish the conservation law suitable for the case of non-isotropic scattering. The results of the rigorous mathematical theory in applying the Lagrangian formalism is used in (Krikorian \& Nikoghossian, 1996) to the one-dimensional transfer problem. Due to the importance of the issue, let us consider it in more detail.

Let us start with the angle-dependent transfer equations for the function $Y$

$$
\pm \eta \frac{d Y(\tau, \pm \eta, \mu)}{d \tau}=-Y(\tau, \pm \eta, \mu)+\frac{\lambda}{2} \int_{-1}^{1} Y\left(\tau, \eta^{\prime}, \mu\right) \mathrm{d} \eta^{\prime}
$$

From these equations one can easily obtain

$$
\eta^{2} \frac{\mathrm{d}^{2} \Phi}{\mathrm{d} \tau^{2}}=-\Phi(\tau, \eta, \mu)-\lambda \int_{0}^{1} \Phi\left(\tau, \eta^{\prime}, \mu\right) \mathrm{d} \eta^{\prime}
$$

where $\Phi(\tau, \eta, \mu)=Y(\tau,+\eta, \mu)+Y(\tau,-\eta, \mu)$.

One may readily check the self-adjointness of this equation so that the variational formulation is admitted. The Lagrangian density $L$ corresponding to Eq. (73) was obtained in (Anderson, 1973)

$$
L\left(\Phi, \Phi^{\prime}, \tau, \eta, \mu\right)=\Phi^{2}+\left(\eta \Phi^{\prime}\right)^{2}-2 \Phi U,
$$

where

$$
U(\tau, \mu)=\frac{\lambda}{2} \int_{0}^{1} \Phi\left(\tau, \eta^{\prime}, \mu\right) \mathrm{d} \eta^{\prime}
$$

In accordance with this result, the Euler-Lagrange equation has a form

$$
\frac{\partial L}{\partial \Phi}-\frac{\mathrm{d}}{\mathrm{d} \tau} \frac{\partial L}{\partial \Phi^{\prime}}+\lambda \int_{0}^{1} \frac{\partial L}{\partial U} \mathrm{~d} \eta^{\prime}=0
$$

One will make sure that insertion of the Lagrangian (74) into (76) yields the transfer equation (73). It is important that both the transfer equation (73) and the Lagrangian density (74) do not depend explicitly on $\tau$, or stated differently, they are form-invariant under infinitesimal transformation

$$
\tau \rightarrow \tau^{\prime}=\tau+\delta \tau, \quad \eta=\eta^{\prime}, \quad \mu=\mu^{\prime},
$$

where the quantity $\delta \tau$ is allowed to be an arbitrary infinitesimal function of $\tau$. This implies that the transformation Eq. (77), i.e. translation of the optical depth, is the symmetry transformation for Eq. (72) and suggests a certain conservation law as follows

$$
\int_{0}^{1}\left[L-\frac{\partial L}{\partial \Phi} \Phi^{\prime}\right] \mathrm{d} \eta=\text { const }
$$

which, in view of (74), takes a form

$$
\int_{0}^{1}\left[\Phi^{2}(\tau, \eta, \mu)-\eta^{2} \Phi^{\prime 2}(\tau, \eta, \mu)-2 U(\tau, \mu) \Phi(\tau, \eta, \mu)\right] \mathrm{d} \eta=\mathrm{const}
$$

or

$$
\int_{0}^{1} Y(\tau, \varsigma, \mu) Y(\tau,-\varsigma, \mu) \mathrm{d} \varsigma=\frac{\lambda}{4}\left(\int_{-1}^{+1} Y(\tau, \varsigma, \mu) \mathrm{d} \varsigma\right)^{2}+\text { const. }
$$


This relation is, in essence, a prototype of the $Q$ - integral obtained by Rybicki (1977). The above considerations imply that by its content the integral (80) is an analog of the momentum conservation law in mechanics and is due to the axes translation transformation.

For semi-infinite atmosphere $Y(\tau, \pm \varsigma, \mu) \rightarrow 0$ as $\tau \rightarrow \infty$ so that, const $=0$, and we arrive at Eq. (53). More general relation for this case Eq. (71) may be derived in treating the problems differing with each other by the value of the parameter $\mu$. It holds everywhere where $\lambda$ does not vary with depth, i.e., if the medium is homogeneous.

The variational formalism suggested in the Byurakan observatory allows not only to elucidate the physical meaning of invariance principle but enables one to derive along with many known results a great number of new relations of great importance for the theory and applications. It allows also to find out some statistical characteristics of the diffusion process in the atmosphere (Nikoghossian, 1997, 2000). Some of the known non-linear relations possess a fairly obvious physical or/and probabilistic meaning and could be written immediately on the base of simple arguments. However, as we shall show below, by no means all of them follow from the variational principle, so they cannot be recognized as invariance relations or, even more, invariance principles.

xii. The polynomial distribution of sources. An important question to be answered is the applicability of the Lagrangian formalism to the transfer problems for atmospheres containing energy sources. Inasmuch as both the diffuse reflection and Milne problems obey the homogeneous integral-differential equation, the suitable quadratic and bilinear integrals are possible to write directly. In the case of the source-containing atmospheres we are led to the inhomogeneous integral-differential equations with appropriate source terms. Generally, the self-adjointness of the transfer equations may be violated so that the direct application of variational principle becomes impossible. Nevertheless, as it was shown by Nikoghossian (1999), in some cases such as the uniformly and exponentially distributed sources this difficulty was get over by using the apparent connection between these two problems treated in Sect. vii and the source-free problem. Now an additional possibility based on the conservation law (79) is appeared to reduce the source-containing problems to the source-free problem. As an example we shall briefly reproduce here the results obtained for the case of polynomial distribution of sources (Nikoghossian, 1999). The first attempt in this direction was made in (Rybicki, 1977). The approach proposed there was successful, however, for polynomials of the degree not higher than second, whereas, as it was shown in the mentioned Nikoghossian's paper, the $Q$-integrals exist for polynomials for arbitrary high order. Let we have the transfer equation of the form

$$
\eta^{2} \frac{\mathrm{d}^{2} \Phi}{\mathrm{d} \tau^{2}}=-\Phi(\tau, \eta)-\lambda \int_{0}^{1} \Phi\left(\tau, \eta^{\prime}\right) \mathrm{d} \eta^{\prime}-2 g(\tau),
$$

where

$$
g(\tau)=(1-\lambda) B(\tau), \quad B(\tau)=\sum_{i=0}^{N} B_{i} \tau^{i} .
$$

where $N$ is the degree of polynomials, $B^{(m)}=d^{m} B / d \tau^{m}$. By introducing the functions

$$
F_{N}(\tau, \eta)=2 \sum_{k=0}^{[N / 2]} B^{(2 k)}(\tau) p_{2 k}(\eta)
$$

where brackets mean the integer part, one can check by direct substitution that $F_{N}$ is a partial solution of Eq.(81) if only $p_{2 k}(\eta)$ are polynomials of the order $2 k(k=1,2, \ldots)$ defined as follows

$$
\begin{aligned}
& p_{2 k}(\eta)=\sum_{i=0}^{k} p_{2 i}(0) \eta^{2(k-i)},(\text { ??) and } \\
& \qquad p_{2 k}(0)=\frac{\lambda}{1-\lambda} \sum_{i=1}^{k} \frac{p_{2(k-i)}(0)}{2 k+1}, p_{0}(\eta)=1 .
\end{aligned}
$$

Having the rule for construction the function (84), one can derive the requisite $Q$-integrals for any source term with polynomial depth-dependence. Some results for small values of $N$ are presented in (Nikoghossian, 1999).

xiii. The group of the RSF (reducible to the source-free) problems. Thus, one may conclude that there exists a group of different frequently occurring radiation transfer problems of astrophysical interest which admit quadratic and bilinear integrals. They can be reduced to the source-free problem. This group includes the Milne's problem, the problem of diffuse reflection (and transmission in the case of the atmosphere of finite optical thickness) as well as the problems with exponential and polynomial laws for the distribution of internal energy sources. An important special case of the last type of problems is the problem of the radiative transfer in an isothermal atmosphere (i.e. in atmosphere with homogeneous distribution of internal sources). This group of problems is characterized at least by three common features. First of all, the invariance principle implies bilinear relations connecting the solutions 
of the listed problems. As it was shown in (Nikoghossian, 2011b), the group of the RSF-problems admits a class of integrals involving quadratic and bilinear moments of the intensity of arbitrarily high orders. Secondly, if the problem can be formulated for finite atmosphere then the principle allows connecting its solution with that of the proper problem for a semi-infinite atmosphere. Finally, knowledge of the $\varphi$-function reduces their solutions to the Volterra-type equation for the source function with the kernel-function (9). The author refers to these problems as the group of RSF-problems.

xiv. Arbitrarily varying sources. For further insight to the point can by given by considering the general treatment of the problem of sources. Consider the transfer of monochromatic radiation through semi-infinite homogeneous and plane-parallel atmosphere that contains energy sources varied arbitrarily with depth. The source function of this problem, as is known, satisfies integral equation

$$
S(\tau)=\frac{\lambda}{2} \int_{0}^{\infty} \operatorname{Ei}(|\tau-t|) S(t) d t+g(\tau)
$$

As it was shown in (Yengibarian, 1972, Yengibarian \& Nikoghossian, 1973b), the solution of this equation is equivalent to the solution of the following coupled pair of the Volterra-type equations

$$
\begin{aligned}
& S(\tau)=\omega(\tau)+\int_{0}^{\tau} L(\tau-t) S(t) d t, \\
& \omega(\tau)=g(\tau)+\int_{\tau}^{\infty} L(\tau-t) \omega(t) d t .
\end{aligned}
$$

As a matter of fact, the set of equations (86), (87) represents a kind of splitting the so-called $\Lambda$-operator (Hubeny, 1982) and may become useful in solving the transfer problems. Note that being applied to this system, the escape probability approach (Rybicky, 1984) leads, as could be expected, to sufficiently accurate results. For instance, when $B=$ const, one may easily find for $\tau=0$ that $\omega=g /(1-\lambda)$ and $S(0)=\omega=B \sqrt{1-\lambda}$. For deep interiors of the atmosphere it yields

$$
\lim _{t \rightarrow \infty} S(\tau)=\lim _{t \rightarrow \infty} \frac{\omega}{L(\tau)+\sqrt{1-\lambda}}=B .
$$

Thus in both cases his approach leads to exact values of the source function, whereas the same approach applied to Eq. (85) gives the rather crude estimate for the surface value: $S(0)=2(1-\lambda) B /(2-\lambda)$. At the same time, it easy to see that the kernel splitting method provides correct values of $S(0)$ for all the treated special RSF-problems. This follows from the fact that in these cases Eq. (87) allows exact analytical solution so that the problems resolve themselves into the solution of a single Volterra-type equation (86) for the source function.

The approach described for the semi-infinite atmosphere is easy to apply to an atmosphere of finite optical thickness. In fact, the generalization of previous results for such atmosphere is attained trivially and resolves itself to determining the proper values of the integration constants. To proceed, we introduce by analogy to the semi-infinite atmosphere the quantities $Y\left(\tau, \pm \eta, \mu ; \tau_{0}\right)$ with similar probabilistic meaning, characterizing the photon's exit from the boundary $\tau=0$ of an atmosphere for the photon moving in direction $\pm \eta$ at depth $\tau$ (angular arguments are referenced with respect to the outward normal to the surface $\tau=0$ ). This implies that

$$
\begin{gathered}
Y\left(0, \eta, \mu ; \tau_{0}\right)=\delta(\eta-\mu), \quad Y\left(\tau_{0}, \eta, \mu ; \tau_{0}\right)=\mu q\left(\mu, \eta, \tau_{0}\right), \\
Y\left(0,-\eta, \mu ; \tau_{0}\right)=\mu \rho\left(\mu, \eta, \tau_{0}\right), \quad Y\left(\tau_{0},-\eta, \mu ; \tau_{0}\right)=0,
\end{gathered}
$$

where

$$
q\left(\mu, \eta, \tau_{0}\right)=\eta^{-1} \delta(\eta-\mu) e^{-\frac{\tau_{0}}{\eta}}+\sigma\left(\mu, \eta, \tau_{0}\right),
$$

is transmittance of the medium. Since the functions $Y\left(\tau, \pm \eta, \mu ; \tau_{0}\right)$ satisfy the partition transfer equations Eqs. (??), the variational principle leads to the quadratic $Q$ - integral Eq. (80) with the value $(\lambda / 4) \psi^{2}\left(\mu, \tau_{0}\right)$ for the constant. It is apparent that with no more effort one may derive many different kind of bilinear and two-point bilinear relations linking with each other different quantities describing the field of radiation in different depths of the finite media of different optical thickness. Such relations for some of the RSF - problems for finite atmosphere are given in (Nikoghossian, 1999).

Of special interest are the nonlinear relations which establish connection between characteristics of the radiation transfer in finite and semi-infinite atmospheres. We limit ourselves to presenting here only two of them which will be mentioned below 


$$
\begin{gathered}
\eta^{\prime} \rho_{\infty}\left(\eta^{\prime}, \eta\right)=-\eta \rho\left(\eta, \eta^{\prime}, \tau\right)+\int_{0}^{1} q(\eta, \varsigma, \tau) Y_{\infty}\left(\tau,-\varsigma, \eta^{\prime}\right) \mathrm{d} \varsigma^{-} \\
-\frac{\lambda}{2} \psi(\eta, \tau) \int_{0}^{1} Y_{\infty}\left(\tau, \varsigma, \eta^{\prime}\right) \mathrm{d} \varsigma-\frac{\lambda}{2} \varphi_{\infty}\left(\eta^{\prime}\right) \varphi(\eta, \tau), \\
Y_{\infty}\left(\tau, \eta, \eta^{\prime}\right)=q\left(\eta, \eta^{\prime}, \tau\right)-\eta \int_{0}^{1} \rho(\eta, \varsigma, \tau) Y_{\infty}\left(\tau,-\varsigma, \eta^{\prime}\right) \mathrm{d} \varsigma- \\
-\frac{\lambda}{2} \varphi(\eta, \tau) \int_{0}^{1} Y_{\infty}\left(\tau, \varsigma, \eta^{\prime}\right) \mathrm{d} \varsigma-\frac{\lambda}{2} \varphi_{\infty}\left(\eta^{\prime}\right) \psi(\eta, \tau),
\end{gathered}
$$

where quantities pertaining to the semi-infinite atmosphere are marked by the sign of infinity.

xv. The nature of some non-linear relations of the radiation transfer theory. Invariant properties of the problems of radiation transfer in homogeneous atmospheres lead to a variety of non-linear relations. They are widely used and are especially efficient when being combined with other methods of solution. In light of the new results presented in the above cited works carried out in the Byurakan observatory, the question arises to what extent certain nonlinear relations in radiative transfer theory are connected with the variational principles resulting from invariance with respect to the translational transformation of the optical depth. Only those relations are implied here, which are used in connection with the invariance principle or with the method of adding layers. As it was shown by Nikoghossian (2009a,b), some nonlinear relations found in the theory sometimes are erroneously considered to be a consequence of the invariance properties of the problem. This question is crucial to the theory of radiative transfer, so it is expedient to summarize here the findings of the above mentioned works.

To begin, it is important to note that, for instance, Eqs. (10) - (13) in deriving the layers adding law have been written without invoking the invariance principle, to which they are, of course, not related. In fact, these formulas remain valid in general cases, when it is inappropriate to speak of invariance properties of the problem. For example, these equations remain in force even when the media are inhomogeneous, with, of course, their polarity property taken into account (see below).

We now rewrite Eqs. (10) - (13) for the three-dimensional plain-parallel homogeneous atmosphere. The initial problem can be re-formulated now in the following way: let a parallel beam of radiation with intensity $I_{0}$ be incident on the boundary $\tau=0$ of a medium at an angle of $\arccos \varsigma$ to its inner normal (Fig. 9). The intensity of the reflected radiation is related to the reflection function $\rho\left(\eta, \varsigma, \tau_{0}\right)$ by $I(0, \eta, \varsigma)=I_{0} \rho\left(\eta, \varsigma, \tau_{0}\right) \varsigma$. In analogous fashion for transmitted radiation we have $I\left(\tau_{0}, \eta, \varsigma\right)=I_{0} q\left(\eta, \varsigma, \tau_{0}\right) \varsigma$, in which we have retained the customary notation $\sigma$ for the diffuse part of the transmitted radiation. For brevity, the dependence of the intensities on the optical thickness of the medium is left out of the arguments. Since the medium can now be regarded as consisting of two parts with respective thicknesses $\tau$ and $\tau_{0}-\tau$, relations analogous to Eqs. (10) - (13) can be written in the form ( $I_{0}$ can be taken equal to unity without loss of generality).

$$
\begin{gathered}
\rho\left(\eta, \varsigma, \tau_{0}\right) \varsigma=\rho(\eta, \varsigma, \tau) \varsigma+I(\tau, \eta, \varsigma) \mathrm{e}^{-\tau / \eta}+\int_{0}^{1} \sigma\left(\eta, \eta^{\prime}, \tau\right) I\left(\tau, \eta^{\prime}, \varsigma\right) \eta^{\prime} \mathrm{d} \eta^{\prime} \\
\sigma\left(\eta, \varsigma, \tau_{0}\right) \varsigma=\sigma\left(\eta, \varsigma, \tau_{0}-\tau\right) \varsigma \mathrm{e}^{-\tau / \eta}+I^{*}(\tau,-\eta, \varsigma) \mathrm{e}^{-\left(\tau_{0}-\tau\right) / \eta}+ \\
\int_{0}^{1} \sigma\left(\eta, \eta^{\prime}, \tau_{0}-\tau\right) I^{*}\left(\tau,-\eta^{\prime}, \varsigma\right) \eta^{\prime} \mathrm{d} \eta^{\prime} \\
I(\tau, \eta, \varsigma)=\rho\left(\eta, \varsigma, \tau_{0}-\tau\right) \varsigma \mathrm{e}^{-\tau / \varsigma}+\int_{0}^{1} \rho\left(\eta, \eta^{\prime}, \tau_{0}-\tau\right) I^{*}\left(\tau,-\eta^{\prime}, \varsigma\right) \eta^{\prime} \mathrm{d} \eta^{\prime} \\
I^{*}(\tau,-\eta, \varsigma)=\sigma(\eta, \varsigma, \tau) \varsigma+\int_{0}^{1} \rho\left(\eta, \eta^{\prime}, \tau\right) I\left(\tau, \eta^{\prime}, \varsigma\right) \eta^{\prime} \mathrm{d} \eta^{\prime},
\end{gathered}
$$

where the diffuse part of the intensity of the downward moving radiation is indicated by an asterisk.

The nonlinear relations written down by Chandrasekhar in the 1950's and called invariance principles by him (Chandrasekhar, 1960) are well known in radiative transfer theory. These relations were examined by Nikoghossian $(2009 \mathrm{a}, \mathrm{b})$ from the standpoint of their possible connection with the invariance principle.

The problem of diffuse reflection and transmission of radiation by a medium of finite optical depth examined by Chandrasekhar was stated as follows: on a medium of optical thickness $\tau_{0}$ in direction $\left(-\mu_{0}, \varphi_{0}\right)$, falls a parallel beam of radiation with flux $\pi F$ per unit area perpendicular to the direction of incidence. The reflection function $S$ and the transmission function $T$ are introduced so that 


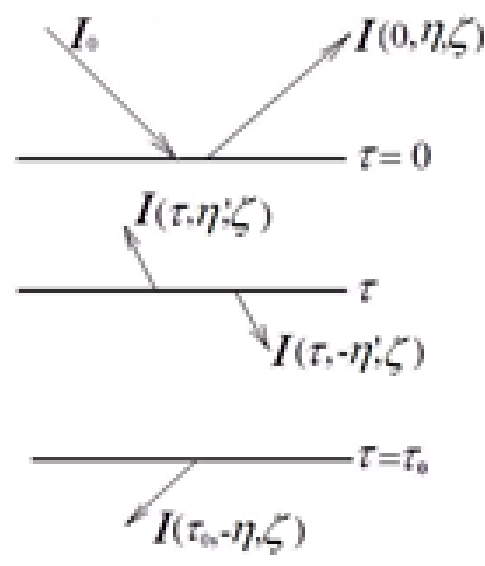

Figure 9: Diffuse reflection of light from a medium of finite thickness.

$$
I(0, \mu, \varphi)=\frac{F}{4 \mu} S\left(\tau_{0}, \mu, \varphi ; \mu_{0}, \varphi_{0}\right), \quad I^{*}\left(\tau_{0},-\mu, \varphi\right)=\frac{F}{4 \mu} T\left(\tau_{0}, \mu, \varphi ; \mu_{0}, \varphi_{0}\right) .
$$

Chandrasekhar wrote down the following four nonlinear equations:

$$
\begin{gathered}
I(\tau, \mu, \varphi)=\frac{F}{4 \mu} e^{-\tau / \mu_{0}} S\left(\tau_{0}-\tau, \mu, \varphi ; \mu_{0}, \varphi_{0}\right)+ \\
\frac{1}{4 \pi \mu} \int_{0}^{2 \pi} \mathrm{d} \varphi \int_{0}^{1} S\left(\tau_{0}-\tau, \mu, \varphi ; \mu^{\prime}, \varphi^{\prime}\right) I^{*}\left(\tau,-\mu^{\prime}, \varphi^{\prime}\right) \mathrm{d} \mu^{\prime}, \\
I^{*}(\tau,-\mu, \varphi)=\frac{F}{4 \mu} T\left(\tau, \mu, \varphi ; \mu_{0}, \varphi_{0}\right)+ \\
\frac{1}{4 \pi \mu} \int_{0}^{2 \pi} \mathrm{d} \varphi \int_{0}^{1} S\left(\tau, \mu, \varphi ; \mu^{\prime}, \varphi^{\prime}\right) I\left(\tau, \mu^{\prime}, \varphi^{\prime}\right) \mathrm{d} \mu^{\prime}, \\
\frac{F}{4 \mu} S\left(\tau_{0}, \mu, \varphi ; \mu_{0}, \varphi_{0}\right)=\frac{F}{4 \mu} S\left(\tau, \mu, \varphi ; \mu_{0}, \varphi_{0}\right)+e^{-\tau / \mu} I(\tau, \mu, \varphi)+ \\
\frac{1}{4 \pi \mu} \int_{0}^{2 \pi} \mathrm{d} \varphi \int_{0}^{1} T\left(\tau, \mu, \varphi ; \mu^{\prime}, \varphi^{\prime}\right) I\left(\tau, \mu^{\prime}, \varphi^{\prime}\right) \mathrm{d} \mu^{\prime}, \\
\frac{F}{4 \mu} T\left(\tau_{0}, \mu, \varphi ; \mu_{0}, \varphi_{0}\right)=\frac{F}{4 \mu} e^{-\tau / \mu_{0}} T\left(\tau_{0}-\tau, \mu, \varphi ; \mu_{0}, \varphi_{0}\right)+e^{-\left(\tau_{0}-\tau\right) / \mu} I(\tau,-\mu, \varphi)+ \\
\frac{1}{4 \pi \mu} \int_{0}^{2 \pi} \mathrm{d} \varphi \int_{0}^{1} T\left(\tau_{0}-\tau, \mu, \varphi ; \mu^{\prime}, \varphi^{\prime}\right) I^{*}\left(\tau,-\mu^{\prime}, \varphi^{\prime}\right) \mathrm{d} \mu^{\prime},
\end{gathered}
$$

We now set ourselves the task of comparing Eqs. (98) - (101) with Eqs. (93) - (96) of the preceding section. To do this we neglect the azimuthal dependence in Eqs. (98) - (101), set $F=1$, switch to the previous notation, and include the following easily verified relation between the corresponding coefficients of reflection and transmission:

$$
(1 / 2) S\left(\tau_{0}, \eta, \varsigma\right)=\eta \varsigma \rho\left(\eta, \varsigma, \tau_{0}\right), \quad(1 / 2) T\left(\tau_{0}, \eta, \varsigma\right)=\eta \varsigma \sigma\left(\eta, \varsigma, \tau_{0}\right)
$$

Then it is easy to show that Eqs. (98) - (101) are the same as Eqs. (95), (96), (93), and (94), respectively. Thus, neither these, nor the previous equations have a direct connection to the invariance principle associated with a translational transformation of the optical depth, and so they cannot be derived from the corresponding conservation law.

These considerations imply that the nonlinear relations discussed above can be divided into two classes. The first includes those formulas which characterize only the radiative transfer process, itself, in a plane parallel atmosphere; these have great generality. Equations (10) - (13), (98) - (101), belong to this class. The second, narrower class includes equations that are a consequence of the invariance properties of the specific transfer problem at hand. Many equations of this type for groups of the RSF-problems have been derived in the first part of this review.

xvi. Inhomogeneous atmosphere. When interpreting the radiation from objects in space it is usually necessary to apply various simplifying assumptions regarding their geometry and physical properties. For example, it is often 
assumed that a radiating medium is homogeneous and stationary, although it clearly has a rather complex structure and is subject to variation in time. This simplifies the problems to a great extent and makes it possible to estimate some characteristics of the radiating medium averaged in some sense. However, the high-resolution observational data available nowadays afford an opportunity for a more detailed investigation of astrophysical objects and analysis of their radiation. This leads to pressure to develop a suitable theory of radiation transfer trough an inhomogeneous atmosphere, providing new efficient methods of computations. Such attempts were made by a number of authors (see e.g., Jefferies, \& Lindsey, 1988; Secchi-Pestelini \& Barletti, 2001; Menkohn, \& Richling, 2002). In connection with solar prominences, it was in (Nikoghossian, S. Pojoga, \& Mouradian, 1997, 1998, 1999; Nikoghossian, \& Mouradian, 2000) treated the effect of physical inhomogeneities related to the distribution of internal energy sources and to geometrical factors. In an analysis of multiple scattering at the line frequencies, the scattering coefficient was usually assumed to be constant inside the radiating volume. It is, however, evident that such an assumption may be rather crude for interpretation of radiation in the optically thick lines. Henceforth under inhomogeneous atmosphere we will mean atmosphere with the scattering coefficient arbitrarily varying with depth, though methods developed in these works remain in force also in the case of variation of other parameters which determine an elementary scattering event and the distribution of primary energy sources.

The complexity of the boundary-value transfer problems makes it necessary to develop appropriate analytical techniques in order to make it somehow easier to get a numerical solution. In each individual case, depending on the initial assumptions about the properties of the medium, of elementary scattering events, etc., specialized methods have been developed. One of the first methods of this type was the method based on invariance principle, i.e., on the symmetry properties of the problem, which avoided the above difficulties in the case of homogeneous atmospheres and allowed to determine the intensity of the emerging radiation without prior knowledge of the radiation field in the entire atmosphere. There has also been a natural drive to find an alternative statement of the classical problems of radiative transfer theory with the aim of reducing them to initial-value problems (so-called Cauchy problems). With the development of high speed electronic computers, research in this area have become especially important in connection with the fact that solving this kind of problem is more suited to the computers' capabilities. Among the first papers in this area, we note those of Bellman (1957) and Sobolev $(1957,1959,1985)$ who developed a method based on extensive use of the "surface" resolvent function. The idea of this approach goes back to Krein's paper (1955).

Attempts to apply the invariance principle in solving the radiation transfer problems for inhomogeneous atmospheres were made by Sobolev (1956) (see also Yanovitskij, 1997). As it was above said, the method of invariant imbedding enables one to readdress standard problems in a way such as to reduce them to the initial value problems. Of the extensive literature in this area, besides the above cited works of Bellman's group of authors, we note also monographs (Scott, 1973; Casti \& Kalaba, 1976).

The method descrybed in Nikoghossian (2004, 2004a,b,c; 2012a,b; 2013a) includes a simple, but, at the same time, universal computational scheme that can be used to determine the radiation field and various characteristics of the scattering process as solutions of corresponding initial-value problems. The idea behind this method developed for solving a given linear problem of the radiation transfer involves a preliminary determination of the global optical properties of an atmosphere - the reflection and transmission coefficients, as well as some other related quantities, for a family of atmospheres with different optical thicknesses. This makes it possible to determine the radiation field inside the $1 D$ inhomogeneous medium without solving any new equations. However, as it was shown below, there exists another route that allows reducing the computational process to ordinary matrix multiplication. Regardless of the initial assumptions, the calculations are easily carried out on modern computers and, most importantly, are numerically stable.

Generalization of the layers adding law for the case of inhomogeneous atmospheres. As it was shown by Nikoghossian (2004), Ambartsumia's arguments and derivations described in Sect.3 for homogeneous atmosphere can be generalised by considering the division of the medium into two parts as a combination of two media generally of different optical properties.

For illustration of the basic idea, it is reproduced here the simplest scalar case involving the transfer of monochromatic radiation in a one-dimensional inhomogeneous atmosphere. As it was shown in (Nikoghossian, 2004), the inhomogeneous atmosphere exhibits the so-called polarity property with respect to the sense of the incoming illumination, i.e., its optical properties in the scalar case are described by three parameters: two reflection coefficients and one transmission coefficient.

Fig.10 is a schematic illustration of two cases where a photon is incident from outside on a composite medium formed as the result of adding two inhomogeneous scattering and absorbing media with optical thicknesses $\tau_{1}$ and $\tau_{2}$.

The formulas for addition in the case shown in the upper part of the figure are 


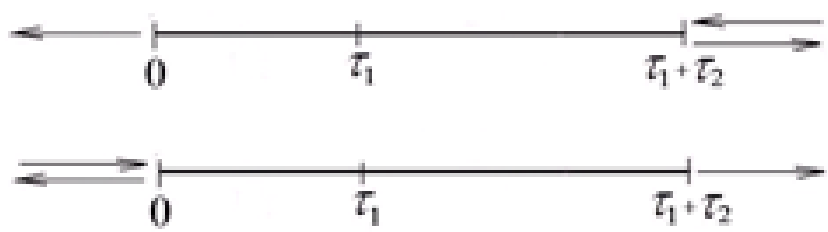

Figure 10: Reflection and transmission of a composite atmosphere.

$$
q\left(\tau_{1}+\tau_{2}\right)=\frac{q\left(\tau_{1}\right) q\left(\tau_{2}\right)}{1-r\left(\tau_{1}\right) \bar{r}\left(\tau_{2}\right)}, \quad r\left(\tau_{1}+\tau_{2}\right)=r\left(\tau_{2}\right)+\frac{r\left(\tau_{1}\right) q^{2}\left(\tau_{2}\right)}{1-r\left(\tau_{1}\right) \bar{r}\left(\tau_{2}\right)},
$$

where the barred values refer the direction inverse to that of radiation incidence. For the reflection coefficient of a composite atmosphere on the left (lower drawing of Fig.10) it was obtained

$$
\bar{r}\left(\tau_{1}+\tau_{2}\right)=\bar{r}\left(\tau_{1}\right)+\frac{r\left(\tau_{1}\right) q^{2}\left(\tau_{2}\right)}{1-r\left(\tau_{1}\right) \bar{r}\left(\tau_{2}\right)} .
$$

It should be noted that, in general, the two components of the composite medium differ one from another not only by optical thickness, but also by the form of the scattering coefficient. Even in the case of the scattering coefficient common for two components, their optical characteristics may differ by the range of variation of this coefficient. But this should not cause confusion in further discussion since henceforth $\tau_{2}$ will be replaced by infinitely thin layer.

Now consider an atmosphere of thickness $\tau_{0}$, where the inhomogeneity stems from variability of the scattering coefficient $\lambda$ with depth, is divided into two parts. Then, the ordinary procedure of taking the limit, when the thickness of one of components tends to zero, leads to equations which coincides with those for homogeneous atmosphere (see, Eq. (16), Eq. (17)). For the inverse illumination one finds

$$
\frac{d \bar{r}}{d \tau_{0}}=\frac{\lambda\left(\tau_{0}\right)}{2} q^{2}\left(\tau_{0}\right),
$$

where we take into account that in the scalar case $\bar{q}=q$ (Nikoghossian, 1999, 2004b),

This is important that $r\left(\tau_{0}\right)$ satisfies a separate equation. It is a Riccati equation and can be solved by one of the standard numerical methods. Note that a fairly high accuracy is obtained even in the simplest case of solving Eq. (17) by the Euler method.

After finding the reflectance, $r\left(\tau_{0}\right)$, the transmission coefficient is determined explicitly using the formula (Nikoghossian, 2011c)

$$
q\left(\tau_{0}\right)=\exp \left[-\int_{0}^{\tau_{0}} \varpi(\tau) d \tau\right]
$$

where

$$
\varpi\left(\tau_{0}\right)=1-\frac{\lambda\left(\tau_{0}\right)}{2}\left[1+r\left(\tau_{0}\right)\right] .
$$

It follows from Eqs. (106) and (105) that as $\tau_{0} \rightarrow \infty, r\left(\tau_{0}\right)$ and $q\left(\tau_{0}\right)$ correspondingly approach $\left(2-\lambda_{\infty}-2 \sqrt{1-\lambda_{\infty}}\right) / \lambda_{\infty}$ and zero, where $\lambda_{\infty}$ is the limiting value of the scattering coefficient.

An alternative approach to the theory of radiation transfer in inhomogeneous media was developed by Nikoghossian $(2012 b)$ by considering the new functions

$$
P\left(\tau_{0}\right)=\frac{1}{q\left(\tau_{0}\right)}, \quad S\left(\tau_{0}\right)=\frac{r\left(\tau_{0}\right)}{q\left(\tau_{0}\right)}=r\left(\tau_{0}\right) P\left(\tau_{0}\right) .
$$

From Eq. (105) it is easy to obtain (see e.g., Nikoghossian, 2004, 2004a, b, c)

$$
\frac{d P}{d \tau_{0}}=\left[1-\frac{\lambda\left(\tau_{0}\right)}{2}\right] P\left(\tau_{0}\right)-\frac{\lambda\left(\tau_{0}\right)}{2} S\left(\tau_{0}\right),
$$

which is the first of the requisite equations. On the other hand, Eq. (17) and (109) imply

$$
\frac{d S}{d \tau_{0}}=\frac{\lambda\left(\tau_{0}\right)}{2} P\left(\tau_{0}\right)-\left[1-\frac{\lambda\left(\tau_{0}\right)}{2}\right] S\left(\tau_{0}\right)
$$

Equations (109) and (110) represent the required linear system of differential equations. It must be supplemented by initial conditions $P(0)=1, S(0)=0$, which follow from the similar conditions for the reflection and transmission 
coefficients. Having solved the system, the quantities $r\left(\tau_{0}\right)$ and $q\left(\tau_{0}\right)$ are readily found from Eq. (108). The system of equations (109) and (110) plays an important role in the theory being put forward.

Note that, if $\lambda\left(\tau_{0}\right)$ is differentiable, Eqs. (109) and (110) may be used to obtain separate differential equations for determining the functions $P\left(\tau_{0}\right)$ and $S\left(\tau_{0}\right)$. Differentiating the mentioned equations, after some simple transformations, we arrive at the following linear differential equations of the second order (Nikoghossian, 2004)

$$
\begin{aligned}
& \frac{d^{2} P}{d \tau_{0}^{2}}-\frac{\lambda^{\prime}}{\lambda} \frac{d P}{d \tau}-\left(1-\lambda-\frac{\lambda^{\prime}}{\lambda}\right) P\left(\tau_{0}\right)=0, \\
& \frac{d^{2} S}{d \tau_{0}^{2}}-\frac{\lambda^{\prime}}{\lambda} \frac{d S}{d \tau}-\left(1-\lambda+\frac{\lambda^{\prime}}{\lambda}\right) S\left(\tau_{0}\right)=0,
\end{aligned}
$$

respectively, with the initial conditions $P(0)=1, P^{\prime}(0)=1-\frac{\lambda(0)}{2}, S(0)=0, S^{\prime}(0)=\frac{\lambda(0)}{2}$.

In the case of homogeneous atmospheres these equations, like the corresponding density of the Lagrangian, do not contain $\tau_{0}$ explicitly (i.e., $\tau_{0}$ is a cyclical coordinate), so that translation of an optical thickness is a symmetry transformation for the system Eqs. (109), (110)). The variation approach proposed in (Krikorian \& Nikoghossian, 1996; Nikoghossian, 1999) for equations of this type indicates that this system of equations allows a conservation law of the form

$$
\left[P\left(\tau_{0}\right)-S\left(\tau_{0}\right)\right]^{2}-(1-\lambda)\left[P\left(\tau_{0}\right)+S\left(\tau_{0}\right)\right]^{2}=\text { const. }
$$

The value of the constant is determined from the initial conditions for the functions $P\left(\tau_{0}\right)$ and $S\left(\tau_{0}\right)$ and equals $\lambda$. It can be expressed in terms of the reflection and transmission coefficients as

$$
\lambda\left[1+r\left(\tau_{0}\right)\right]^{2}-4 r\left(\tau_{0}\right)=\lambda q^{2}\left(\tau_{0}\right) .
$$

If the explicit dependence of $\lambda$ on $\tau_{0}$ is known, then Eqs. (111) and (112) can be solved using one of the available computational schemes for the Cauchy problem for linear differential equations. In order to clarify the particularly mathematical aspect of these results, we must point out the relationship of these equations to Riccati equations. Thus, for example, solving system of Eqs. (109), (110) is equivalent to solving the second order differential equation

$$
F^{\prime \prime}\left(\tau_{0}\right)-\left[1-\lambda\left(\tau_{0}\right)\right] F\left(\tau_{0}\right)=0
$$

For the function $F\left(\tau_{0}\right)=P\left(\tau_{0}\right)+S\left(\tau_{0}\right)$. The initial conditions will obviously be $F(0)=F^{\prime}(0)=1$. On the other hand, it is well known that the substitution $u=F^{\prime} / F$ yields the following Riccati equation

$$
u^{\prime}\left(\tau_{0}\right)+u^{2}\left(\tau_{0}\right)=1-\lambda\left(\tau_{0}\right) .
$$

Equations (111), (112) also are reducible to this type of equation. In some cases, solving this system reduces to finding the solution of ordinary differential equations of known types, whose solutions are expressed in terms of special and elementary functions.

One criticism sometimes raised about the applicability of the methods of adding layers and invariant imbedding is that the latter are supposedly not effective for determining the radiation field inside a medium. Here we show that, in fact, these methods make it easy to determine this field as well as a number of other quantities which describe the process of multiple scattering inside a medium.

One can now write the transfer equations in terms of $U\left(\tau, \tau_{0}\right)$ and $V\left(\tau, \tau_{0}\right)$, which represent the probabilities that photon will move at the optical depth $\tau$ in the direction of decreasing and increasing optical depths, respectively (see Fig.11):

$$
\begin{aligned}
& \frac{d U}{d \tau}=\left[1-\frac{\lambda(\tau)}{2}\right] U\left(\tau, \tau_{0}\right)-\frac{\lambda(\tau)}{2} V\left(\tau, \tau_{0}\right), \\
& \frac{d V}{d \tau}=\frac{\lambda(\tau)}{2} U\left(\tau, \tau_{0}\right)-\left[1-\frac{\lambda(\tau)}{2}\right] V\left(\tau, \tau_{0}\right),
\end{aligned}
$$

These equations satisfy the boundary conditions $U\left(\tau_{0}, \tau_{0}\right)=1, V\left(0, \tau_{0}\right)=0$. In classical astrophysical problems, the transfer equations are usually reduced to just these kind of boundary-value problems. It is easy to see on comparing the system of Eqs. (117) and (118) with Eqs. (109) and (110) that, as functions of optical depth, the functions $U$ è $V$ satisfy the same equations as the functions $P$ è $S$ of the optical thickness. However, in the first case, we have to deal with a boundary-value problem, and in the second, with an initial-value problem. 

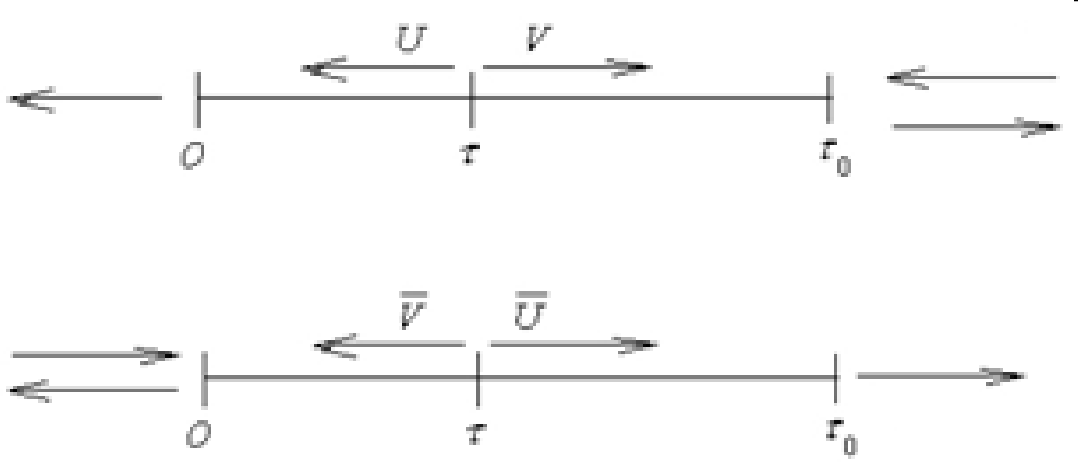

Figure 11: Schematic illustration of radiative transfer in a one-dimensional medium.

Based on some simple physical arguments it is easy to show that

$$
q\left(\tau_{0}\right)=q(\tau) U\left(\tau, \tau_{0}\right), \quad V\left(\tau, \tau_{0}\right)=r(\tau) U\left(\tau, \tau_{0}\right) .
$$

These equations follow mathematically by comparing the conditions at the boundary $\tau=0, U\left(0, \tau_{0}\right)=q\left(\tau_{0}\right)$ and $V\left(0, \tau_{0}\right)=0$, with the same conditions for the functions $P$ and $S$. In fact, these equations need no proof, since the addition rules for the reflection and transmission coefficients have been derived from them.

Eq. (119) yields

$$
U\left(\tau, \tau_{0}\right)=\exp \left[-\int_{\tau}^{\tau_{0}} \varpi\left(\tau^{\prime}\right) d \tau^{\prime}\right], \quad V\left(\tau, \tau_{0}\right)=r(\tau) \exp \left[-\int_{\tau}^{\tau_{0}} \varpi\left(\tau^{\prime}\right) d \tau^{\prime}\right] .
$$

Thus, for determining the radiation field inside a medium it is sufficient to first determine the reflectance of a family of atmospheres by solving Eq. (106). It should be noted that, as is clear from the above formulas, the variables $\tau$ and $\tau_{0}$ in expressions for $U$ and $V$ are separated:

$$
U\left(\tau, \tau_{0}\right)=q\left(\tau_{0}\right) P(\tau), \quad V\left(\tau, \tau_{0}\right)=q\left(\tau_{0}\right) S(\tau) .
$$

These equations follow mathematically from a comparison of the conditions at the boundary, $U\left(0, \tau_{0}\right)=q\left(\tau_{0}\right)$ and $V\left(0, \tau_{0}\right)=0$, with the same conditions for the functions $P$ and $S$.

We see that the radiation field inside the atmosphere is determined entirely by the reflectance and transmittance of its individual parts. In other words, knowledge of the global optical properties for a family of atmospheres with different optical thicknesses, but with a given type of inhomogeneity, can be used for directly determining the radiation intensity at an arbitrary optical depth, without solving any new equations.

Furthermore, the fact that the variables in $U$ and $V$ are separable makes it substantially easier to find these intensities, since the required solution can be obtained immediately for a family of atmospheres with different optical thicknesses. Equations (121) clarify certain features of the radiation field in an atmosphere. Thus, for example, they imply that the radiation fluxes in both directions at some depth $\tau$ in atmospheres with different optical thicknesses are proportional to the transmittance of these atmospheres, while the intensities at different depths inside a given atmosphere are proportional to the corresponding opacity $q(\tau)$.

Knowledge of $r$ makes it possible to find explicit solutions for a whole series of problems that are frequently encountered in astrophysical applications. Here we consider a few of them.

a. Internal energy sources. Suppose that an inhomogeneous atmosphere contains energy sources of power $B(\tau)$ and it is required to determine the intensity of the radiation emerging from the medium and the radiation field within the medium. If we denote the intensities emerging from the medium through the boundaries $\tau=\tau_{0}$ and $\tau=0$ by $I_{1}\left(\tau_{0}\right)$ and $I_{2}\left(\tau_{0}\right)$, then, after some simple reasoning, we can write (Nikoghossian, 2004b)

$$
\begin{gathered}
I_{1}\left(\tau_{0}\right)=\frac{1}{2} q\left(\tau_{0}\right) \int_{0}^{\tau_{0}}[1+r(\tau)] B(\tau) d \tau / q(\tau), \\
I_{2}\left(\tau_{0}\right)=\frac{1}{2} \int_{0}^{\tau_{0}}\left[\lambda(\tau) I_{1}(\tau)+B(\tau)\right] q(\tau) d \tau .
\end{gathered}
$$

Knowledge of the latter is sufficient for determining the intensities $I^{+}\left(\tau, \tau_{0}\right), I^{-}\left(\tau, \tau_{0}\right)$ of the radiation directed, respectively, toward decreasing and increasing depths, i.e., 


$$
\begin{aligned}
I^{+}\left(\tau, \tau_{0}\right) & =\left[I_{2}\left(\tau_{0}\right)-I_{2}(\tau)\right] / q(\tau), \\
I^{-}\left(\tau, \tau_{0}\right) & =r(\tau) I^{+}\left(\tau, \tau_{0}\right)+I_{1}(\tau) .
\end{aligned}
$$

b. Average number of scattering events. We introduce the notations $N_{r}\left(\tau_{0}\right), N_{q}\left(\tau_{0}\right)$ for the MNSE, respectively, for two categories of photons: reflected and transmitted. It has been shown by (Nikoghossian, 2004b) that these quantities can be completely expressed in terms of the above reflection and transmission coefficients for the family of atmospheres by

$$
\begin{aligned}
& N_{r}\left(\tau_{0}\right)=\frac{1}{2} \frac{q^{2}\left(\tau_{0}\right)}{r\left(\tau_{0}\right)} \int_{0}^{\tau_{0}} \lambda(\tau)\left[1+r^{2}(\tau)\right] \frac{d \tau}{q^{2}(\tau)}, \\
& N_{q}\left(\tau_{0}\right)=\frac{1}{2} \int_{0}^{\tau_{0}} \lambda(\tau)\left\{1+r(\tau)\left[1+N_{r}(\tau)\right]\right\} d \tau .
\end{aligned}
$$

c. Reflection from the opposite boundary. For completeness, here we also give the formula for the reflection coefficient of this medium when it is illuminated from the side of the boundary $\tau=0$ in Fig.10. It was shown (Nikoghossian, 2004, 2004a,b,c), that

$$
\bar{r}\left(\tau_{0}\right)=\frac{1}{2} \int_{0}^{\tau_{0}} \lambda(\tau) q^{2}(\tau) d \tau=\frac{1}{2} \int_{0}^{\tau_{0}} \lambda(\tau) e^{-2 \int_{0}^{\tau} \varpi\left(\tau^{\prime}\right) d \tau^{\prime}} d \tau
$$

Thus, we can note the major conclusion of this section: in order to solve the scalar problem of the radiation transfer in inhomogeneous atmosphere it is enough to know its reflectivity, since all the other quantities of interest are found explicitly.

\section{xvii. Group-theoretical description of radiative transfer.}

Media composition groups. Scalar case. Ambarsumian's method of addition of layers was further developed by Nikoghossian (2011a; 2014a,b; 2016, 2019), where, for the first time, the group theory was applied to solve the problems of radiative transfer in inhomogeneous absorbing and scattering atmospheres. It was introduced the concept of a composition or transformation of scattering and absorbing atmospheres, which involves the adding of an additional atmosphere to an initial one in the general case of an inhomogeneous atmosphere (or removing some part from an initial atmosphere). It was assumed that the added or subtracted parts do not contain primary energy sources. The transformations induced in this way form a group if the group product is taken to mean the resultant of two successive transformations. It is easy to see that the other conditions for formation of a group are satisfied. In particular, the role of a unit element is the identity transformation, which leaves the initial atmosphere unchanged, and the inverse elements are transformations which reverse the effect of one or another already performed transformation. The associativity of the group product is evident. This group of transformations was referred to as the GN2 group. It is easy to see that, in general, it is not commutative. Among this type of groups, an important role is played by groups associated with the formation of a multicomponent atmosphere. In that case, the transformation is taken to mean the addition (or removal) of a homogeneous medium characterized by an optical thickness and a scattering coefficient $\lambda$. This group (referred to as GNH2) is a two-parameter, noncommutative group. The special case where $\lambda$ is the same for all the added or removed media (group GH2) yields the case of homogeneous atmospheres. In this case, the group is obviously commutative, i.e., is abelian (Wigner, 1961). It is also a one-parameter, infinite, and continuous group.

Every homogeneous medium of optical thickness $\tau_{0}$ can be characterized by two quantities, the reflection $r\left(\tau_{0}\right)$ and transmission $q\left(\tau_{0}\right)$ coefficients, which have a probabilistic meaning. At the same time, it has been shown (Nikoghossian 2004, 2004a) that an inhomogeneous atmosphere has a polarity property, i.e., its optical properties are described by three parameters: two reflection coefficients and one transmission coefficient. Turning again to Fig. 10 and the layers adding laws Eq. (103), Eq. (104), one can rewrite these laws in terms of functions $P, S, \bar{S}$. These were derived in (Nikoghossian, 2011a) and read as

$$
\begin{gathered}
P\left(\tau_{1}+\tau_{2}\right)=P\left(\tau_{1}\right) P\left(\tau_{2}\right)-S\left(\tau_{1}\right) \bar{S}\left(\tau_{2}\right), \\
S\left(\tau_{1}+\tau_{2}\right)=P\left(\tau_{1}\right) S\left(\tau_{2}\right)+S\left(\tau_{1}\right) M\left(\tau_{2}\right),
\end{gathered}
$$

where $M(\tau)=[1-S(\tau) S(\tau)] / P(\tau)$. Similarly,

$$
\bar{S}\left(\tau_{1}+\tau_{2}\right)=P\left(\tau_{2}\right) \bar{S}\left(\tau_{1}\right)+\bar{S}\left(\tau_{2}\right) M\left(\tau_{1}\right) .
$$

It can be confirmed by direct testing that there is also an addition law for $M(\tau)$, 


$$
M\left(\tau_{1}+\tau_{2}\right)=M\left(\tau_{1}\right) M\left(\tau_{2}\right)-S\left(\tau_{1}\right) \bar{S}\left(\tau_{2}\right)
$$

On introducing the matrices

$$
A(\tau)=\left(\begin{array}{cc}
P(\tau) & -\bar{S}(\tau) \\
S(\tau) & M(\tau)
\end{array}\right)
$$

it is easy to confirm that they also constitute a group and form a representation of the group GN2. In fact, each element of the group GN2 corresponds to a transformation $T(g)$,

$$
\left(\begin{array}{c}
P\left(\tau_{1}+\tau_{2}\right) \\
S\left(\tau_{1}+\tau_{2}\right)
\end{array}\right)=\left(\begin{array}{cc}
P\left(\tau_{2}\right) & -\bar{S}\left(\tau_{2}\right) \\
S\left(\tau_{2}\right) & M\left(\tau_{2}\right)
\end{array}\right)\left(\begin{array}{c}
P\left(\tau_{1}\right) \\
S\left(\tau_{1}\right)
\end{array}\right)
$$

if the medium is illuminated from the right, and $T^{\prime}(g)$,

$$
\left(\begin{array}{c}
P\left(\tau_{1}+\tau_{2}\right) \\
\bar{S}\left(\tau_{1}+\tau_{2}\right)
\end{array}\right)=\left(\begin{array}{cc}
P\left(\tau_{1}\right) & -S\left(\tau_{1}\right) \\
\bar{S}\left(\tau_{1}\right) & M\left(\tau_{1}\right)
\end{array}\right)\left(\begin{array}{c}
P\left(\tau_{2}\right) \\
\bar{S}\left(\tau_{2}\right)
\end{array}\right)
$$

in the opposite case.

In addition, the group product $g_{1} \otimes g_{2}$ corresponds to the matrix product $A\left(\tau_{1}+\tau_{2}\right)=A\left(\tau_{2}\right) A\left(\tau_{1}\right)$, i.e., $T\left(g_{1} \otimes g_{2}\right)=$ $T\left(g_{2}\right) T\left(g_{1}\right)$, for illumination from the right and $\tilde{A}\left(\tau_{1}+\tau_{2}\right)=\tilde{A}\left(\tau_{1}\right) \tilde{A}\left(\tau_{2}\right)$, i.e., $T^{\prime}\left(g_{1} \otimes g_{2}\right)=T^{\prime}\left(g_{1}\right) T^{\prime}\left(g_{2}\right)$, for illumination from the left. (Here a tilde denotes the transposed matrix.) The identity transformation obviously corresponds to the unit matrix: $T(e)=\mathbf{E}$ and $T,(e)=\mathbf{E}$. The matrix $\mathbf{A}(\tau)$ is nonsingular (its determinant equals 1), so inverse matrices exist, with

$$
A^{-1}(\tau)=\left(\begin{array}{cc}
M(\tau) & S(\tau) \\
-\bar{S}(\tau) & P(\tau)
\end{array}\right), \tilde{A}^{-1}(\tau)=\left(\begin{array}{cc}
M(\tau) & \bar{S}(\tau) \\
-S(\tau) & P(\tau)
\end{array}\right)
$$

These two group representations are isomorphic, since the correspondence between the groups GN2 and $T(g)$, as well as between GN2 and $T^{\prime}(g)$, are mutually unique.

The vector-matrix case. Up to now the discussion was restricted to the simplest scalar problem for onedimensional media. It was shown by Nikoghossian $(2014 \mathrm{a}, \mathrm{b}, \mathrm{c})$ that the results can be generalized to the matrix case, which covers a wide range of astrophysical problems in which the spatial and frequency distributions of the radiation field are taken into account. Besides its purely theoretical significance, this generalization is of great practical importance since it makes it possible to develop new methods for numerical solution of many extremely complicated astrophysical problems. In particular, these include the formation of spectral lines in media with various kinds of inhomogeneities, particularly, in those with fine structure that can be observed with the aid of powerful modern instrumentation.

In general, the adding media can differ from one another in optical thickness, as well as in the character of their inhomogeneity related to variations in different parameters that control elementary scattering event, such as the absorption profile, scattering coefficient, scattering indicatrix, frequency distribution of the radiation, degree of polarization, etc. The matrix case described in the above-mentioned works includes both the diffusion of radiation in a three-dimensional plane-parallel medium, and in a one-dimensional medium where the frequency redistribution of the radiation is taken into account. The results were presented for the last of these two cases with partial frequency redistribution where the inhomogeneity of the atmosphere resulting from by variations in the scattering coefficient within it. As above, the composition or transformation of absorbing and scattering atmospheres was taken to mean adding another, generally, inhomogeneous atmosphere to one of the sides of an initial atmosphere. These transformations form a group if the group product (a binary operation) in it signifies the result of two successive transformations. Everything stated above for 1D case relating to the conditions for formation of a group in the scalar problem remains in force in the matrix case examined here. This generalized version of the composition group was referred nominally to as a $\mathrm{GN}(2, \mathrm{C})$ group which, like the $\mathrm{GN}(2)$ group in the scalar problem is infinite and, in general, noncommutative. The set of atmospheres obtained as a result of a described composition will evidently consist of multicomponent atmospheres with inhomogeneous components. There are two subgroups of $\mathrm{GN}(2, \mathrm{C})$, both of which are of independent interest. The first is related to the composition of homogeneous media, which differ from one another not only in thickness, but also in several optical properties that characterize the diffusion process in it. These groups referred to as $\mathrm{GNH}(2, \mathrm{C})$ are noncommutative and two, three-, or multicomponent, depending on the number of parameters that vary on going from one medium to another. This sort of multicomponent media, in which each component is homogeneous and differs from the others in its optical characteristics, can serve as a prototype for an entire series of real radiative media that are often encountered in astrophysical applications. 


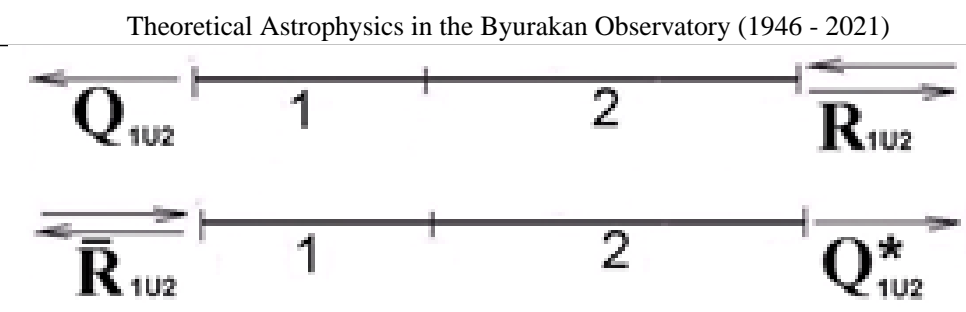

Figure 12: Reflection and transmission in an inhomogeneous two-component atmosphere.

The second subgroup is contained in the first and refers to the case when the added layers have the same properties and differ from one another only in their thicknesses (group $\mathrm{GH}(2, \mathrm{C})$ ). Obviously, any transformation in this case yields only homogeneous media. This kind of group is commutative (abelian), one-parameter, infinite, and continuous with the continuously varying optical thickness.

Fig. 12 demonstrates a configuration consisting of two media, which differ from one another generally in optical thickness and in the functional behavior of their parameters which determine an elementary scattering event. This means that both components are inhomogeneous, so they have the property of polarity. As opposed to the scalar case, the reflection and transmission coefficients now depend on frequency (and also on direction in three-dimensional problems), as well as on thickness; thus, it is convenient to write them down in operator form. These coefficients are denoted, respectively, by $\mathbf{R}_{i}, \mathbf{Q}_{i}(i=1,2)$ when the medium is illuminated from the right (top drawing in Fig. 8) and by $\mathbf{R}_{i}$ and $\mathbf{Q}_{i}$ when the medium is illuminated from the left (bottom drawing). We recall also that $\mathbf{Q}_{i}=\mathbf{Q}_{i}^{*}$, where the asterisk denotes the transposed matrix. Thus, in the following discussion one shall adhere to the notation $\mathbf{Q}_{i}^{*}$.

Along with the transmission coefficients $\mathbf{Q}_{i}$, their inverse matrices $\mathbf{P}_{i}=\mathbf{Q}_{i}^{-1}, \mathbf{P}_{i}^{*}=\left(\mathbf{Q}_{i}^{*}\right)^{-1}$, (they exist because the matrix $\mathbf{Q}_{i}$ is nondegenerate) the matrices $\mathbf{S}_{i}=\mathbf{R}_{i} \mathbf{P}_{i}$ and $\overline{\mathbf{S}}_{\mathbf{i}}=\mathbf{P}_{\mathbf{i}} \overline{\mathbf{R}}_{\mathbf{i}}$ play an important role in the developed theory The matrix analogs of addition laws Eqs. $(129,130)$ are written as follows

$$
\mathbf{P}_{1 \cup 2}=\mathbf{P}_{2} \mathbf{P}_{1}-\overline{\mathbf{S}}_{2} \mathbf{S}_{1}, \quad \mathbf{S}_{1} \cup \mathbf{2}=\mathbf{S}_{2} \mathbf{P}_{1}+\mathbf{M}_{2} \mathbf{S}_{1}
$$

where $\mathbf{M}=\mathbf{Q}^{*}-\mathbf{S} \overline{\mathbf{R}}$.

Using the concept of supermatrices (Berezin, 1986; Wigner, 1961), Eqs. (137) can be combined and written in the form

$$
\left(\begin{array}{l}
\mathbf{P}_{1} \cup 2 \\
\mathbf{S}_{1} \cup 2
\end{array}\right)=\left(\begin{array}{cc}
\mathbf{P}_{2} & -\overline{\mathbf{S}}_{2} \\
\mathbf{S}_{2} & \mathbf{M}_{2}
\end{array}\right)\left(\begin{array}{l}
\mathbf{P}_{1} \\
\mathbf{S}_{1}
\end{array}\right)
$$

It is introduced the notation

$$
\tilde{\mathbf{A}}=\left(\begin{array}{cc}
\mathbf{P} & -\overline{\mathbf{S}} \\
\mathbf{S} & \mathbf{M}
\end{array}\right),
$$

where the tilde means that a quantity is a supermatrix (or, as it is still customarily called, a cell or block matrix).

One can see that the supermatrices $\tilde{\mathbf{A}}_{\mathbf{i}}$ also form a group and are representations of the group $\mathrm{GN}(2, \mathrm{C})$ that maps it in a linear supervector space. It is important to note that the technique for composition of absorbing and scattering media described by $\tilde{\mathbf{A}}_{\mathbf{i}}$ (the transformation $g$ ) does not depend on that what of some particular parameters is responsible for inhomogeneity. Thus, these results apply equally to the media with components that have of different kind inhomogeneity.

It is easy to understand that the transformation Eq.(138) only provides a partial answer about the optical properties of the resultant atmosphere. It can be used to determine the reflectivity and transmission of a medium only from the side on which it is illuminated; this can be regarded as sufficient only in the case of a homogeneous atmosphere. To find the lacking properties of the resultant atmosphere, we proceed as follows. It is evident that adding new media twice on the right to an initial layer is equivalent to taking the product of matrices (139). This yields the addition rules for $\overline{\mathbf{S}}$ and $\mathbf{M}$,

$$
\overline{\mathbf{S}}_{\mathbf{1} \cup \mathbf{2}}=\mathbf{P}_{\mathbf{2}} \overline{\mathbf{S}}_{\mathbf{1}}+\overline{\mathbf{S}}_{\mathbf{2}} \mathbf{M}_{\mathbf{1}}, \quad \mathbf{M}_{\mathbf{1} \cup \mathbf{2}}=\mathbf{M}_{\mathbf{2}} \mathbf{M}_{\mathbf{1}}-\mathbf{S}_{\mathbf{2}} \overline{\mathbf{S}}_{\mathbf{1}} .
$$

Equations (xv.12) can now be written in vector-matrix form as

$$
\left(\begin{array}{c}
\mathbf{M}_{1 \cup 2} \\
\overline{\mathbf{S}}_{1 \cup 2}
\end{array}\right)=\left(\begin{array}{cc}
\mathbf{M}_{2} & -\mathbf{S}_{2} \\
\overline{\mathbf{S}}_{2} & \mathbf{P}_{2}
\end{array}\right)\left(\begin{array}{c}
\mathbf{M}_{1} \\
\overline{\mathbf{S}}_{1}
\end{array}\right)
$$

Thus, we arrive at yet another representation of the transformation $g$ by means of the supermatrix 


$$
\tilde{\mathbf{B}}=\left(\begin{array}{cc}
\mathbf{M} & -\mathbf{S} \\
\overline{\mathbf{S}} & \mathbf{P}
\end{array}\right) .
$$

The notations $T$ ( $g$ ) and $\bar{T}(g)$ are introduced to denote the above representations of the group GN(2,C) corresponding to transformations of the initial medium from the right. Then the group products $g_{1} \otimes g_{2}$ will correspond to the supermatrix products $\tilde{\mathbf{A}}_{\mathbf{1} \cup \mathbf{2}}=\tilde{\mathbf{A}} \mathbf{2}_{\mathbf{A}} \tilde{\mathbf{1}}_{\mathbf{1}}$ and $\tilde{\mathbf{B}}_{\mathbf{1} \cup \mathbf{2}}=\tilde{\mathbf{B}}_{\mathbf{1}} \tilde{\mathbf{B}}_{\mathbf{2}}$, i.e., $\left.T\left(g_{1} \otimes g_{2}\right)=T\left(g_{2}\right) T\left(g_{1}\right)\right)$ and $\bar{T}\left(g_{1} \otimes g_{2}\right)=\bar{T}\left(g_{2}\right) \bar{T}\left(g_{1}\right)$. The identity transformation $e$ corresponds to the supermatrix

$$
\tilde{\mathbf{E}}=\left(\begin{array}{ll}
\mathbf{I} & \mathbf{0} \\
\mathbf{0} & \mathbf{I}
\end{array}\right),
$$

where $\mathbf{I}$ is the unit matrix.

The representations Eq.(139) and Eq.(142) found above are isomorphic to the group GN(2,C), since the correspondence between this group and $T(g), \bar{T}(g)$ is mutually unique and the product of any two elements from GN $(2, \mathrm{C})$ corresponds to matrix multiplications of the corresponding representations of this group. These two representations can be combined and written in the form of the reducible representation

$$
\left(\begin{array}{c}
\mathbf{P}_{1 \cup 2} \\
\mathbf{S}_{1 \cup 2} \\
\mathbf{M}_{1 \cup 2} \\
\overline{\mathbf{S}}_{1 \cup 2}
\end{array}\right)=\left(\begin{array}{cccc}
\mathbf{P}_{2} & -\overline{\mathbf{S}}_{2} & \mathbf{0} & \mathbf{0} \\
\mathbf{S}_{2} & \mathbf{M}_{2} & \mathbf{0} & \mathbf{0} \\
\mathbf{0} & \mathbf{0} & \mathbf{M}_{2} & -\mathbf{S}_{2} \\
\mathbf{0} & \mathbf{0} & \overline{\mathbf{S}}_{2} & \mathbf{P}_{2}
\end{array}\right)\left(\begin{array}{c}
\mathbf{P}_{1} \\
\mathbf{S}_{1} \\
\mathbf{M}_{1} \\
\overline{\mathbf{S}}_{1}
\end{array}\right),
$$

with $\mathbf{P}(\mathbf{0})=\mathbf{M}(\mathbf{0})=\mathbf{I}, \mathbf{S}(\mathbf{0})=\overline{\mathbf{S}}(\mathbf{0})=\mathbf{0}$.

Therefore, it is concluded that the ordinary matrix multiplication can be used to determine the quantities expressing the reflectivity and transmittance of a composite atmosphere if these parameters of its components are known. Evidently, in the case of a homogeneous medium, it is sufficient to consider the transformation Eq. (138) instead of Eq. (144).

When the composite medium is illuminated from its left boundary (bottom drawing of Fig.12), the composition transformation reads

$$
\left(\begin{array}{l}
\mathbf{P}_{1}^{*} \cup \mathbf{2} \\
\overline{\mathbf{S}}_{\mathbf{1} \cup \mathbf{2}}^{*}
\end{array}\right)=\left(\begin{array}{cc}
\mathbf{P}_{1}^{*} & -\mathbf{S}_{1}^{*} \\
\overline{\mathbf{S}}_{1}^{*} & \mathbf{M}_{1}^{*}
\end{array}\right)\left(\begin{array}{c}
\mathbf{P}_{2}^{*} \\
\overline{\mathbf{S}}_{2}^{*}
\end{array}\right) \text {. }
$$

Finally, instead of Eq. (141), it is obtained

$$
\left(\begin{array}{c}
\mathbf{M}_{1}^{*} \cup \mathbf{2} \\
\mathbf{S}_{1 \cup 2}^{*}
\end{array}\right)=\left(\begin{array}{cc}
\mathbf{M}_{1}^{*} & -\overline{\mathbf{S}}_{1}^{*} \\
\mathbf{S}_{1}^{*} & \mathbf{P}_{1}^{*}
\end{array}\right)\left(\begin{array}{c}
\mathbf{M}_{2}^{*} \\
\mathbf{S}_{2}^{*}
\end{array}\right)
$$

Thus, there was found two new representations of the group $\mathrm{GN}(2, \mathrm{C})$ that describe the transformation of the global optical characteristics of a composite atmosphere illuminated on its left boundary $\tau=0$

$$
\tilde{\mathbf{C}}=\left(\begin{array}{cc}
\mathbf{P}^{*} & -\mathbf{S}^{*} \\
\overline{\mathbf{S}}^{*} & \mathbf{M}^{*}
\end{array}\right) . \quad \tilde{\mathbf{D}}=\left(\begin{array}{cc}
\mathbf{M}^{*} & -\overline{\mathbf{S}}^{*} \\
\mathbf{S}^{*} & \mathbf{P}^{*}
\end{array}\right) .
$$

As it was proofed by Nikoghossian (2019), the superdeterminants (Berezinians) of all the supermatrices $\tilde{\mathbf{A}}, \tilde{\mathbf{B}}, \tilde{\mathbf{C}}$ and $\tilde{\mathbf{D}}$ are equal to unity, so that both two-sided inverse matrices of them exist. Here are these matrices

$$
\tilde{\mathbf{A}}^{-1}=\left(\begin{array}{cc}
\mathbf{M}^{*} & \overline{\mathbf{S}}^{*} \\
-\mathbf{S}^{*} & \mathbf{P}^{*}
\end{array}\right), \quad \tilde{\mathbf{B}}^{-1}=\left(\begin{array}{cc}
\mathbf{P}^{*} & \mathbf{S}^{*} \\
-\overline{\mathbf{S}}^{*} & \mathbf{M}^{*}
\end{array}\right), \quad \tilde{\mathbf{C}}^{-1}=\left(\begin{array}{cc}
\mathbf{M} & \mathbf{S} \\
-\overline{\mathbf{S}} & \mathbf{P}
\end{array}\right) . \quad \tilde{\mathbf{D}}^{-1}=\left(\begin{array}{cc}
\mathbf{P} & \overline{\mathbf{S}} \\
-\mathbf{S} & \mathbf{M}
\end{array}\right) .
$$

These supermatrices are fundamental in the developed theory since they establish the laws of transformation of global optical characteristics in composing scattering and absorbing media. All of them have a number of common properties: they are non-singular (non-degenerate) and have invariant superdeterminants equal to unity. In other words, the only global invariant during composition of media of arbitrary optical thickness is the superdeterminant of the corresponding transformation operator. Physically, this is a consequence of the invertibility property of optical phenomena. This result can be illustrated using the geometric significance of the determinant of an ordinary twodimensional matrix (Fig. 13).

The theory developed in (Nikoghossian, 2011, 2014a] has been applied to the problem of diffusive reflection of line radiation from an atmosphere of finite thickness with partial frequency redistribution. Transformations of the group $\mathrm{GNH}(2, \mathrm{C})$ make it possible to construct a multicomponent atmosphere with a certain optical thickness $\tau_{0}$, the 


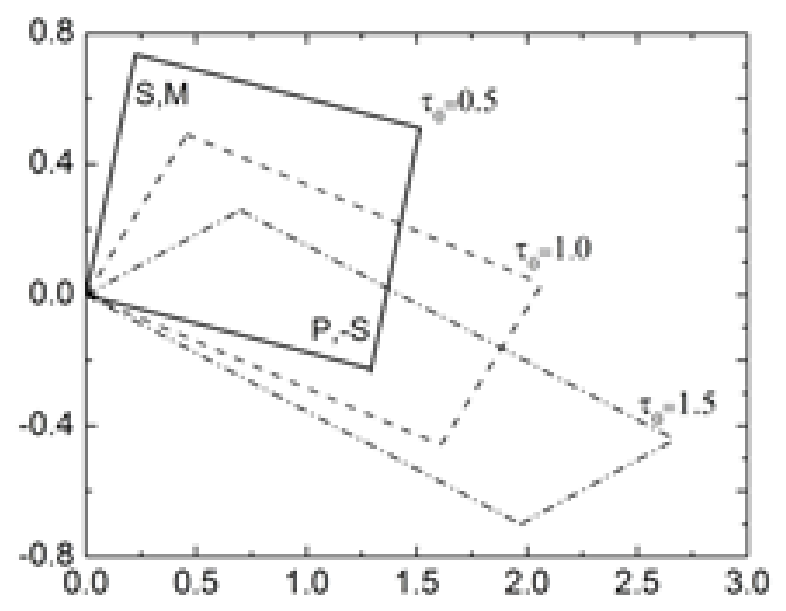

Figure 13: Geometrical interpretation of the determinant of a scalar matrix $\tilde{A}$ for several values of the optical thickness.

components of which can be distinguished by their physical characteristics. As an example, it was examined a onedimensional, inhomogeneous medium illuminated on the side at a boundary $\tau=\tau_{0}$. It was assumed that medium is composed of components of equal and quite small thickness, which are characterized by constant values of the scattering coefficient $\lambda$ such that it can be regarded as a continuous function of optical depth in the limit when the thickness of the components goes to zero. For the infinitesimal operator of the group $\tilde{A}$ with the parameter equal to $\tau_{0}$, it was obtained

$$
\tilde{\Xi}\left(\tau_{\mathbf{0}}\right)=\lim _{\boldsymbol{\Delta} \tau_{\mathbf{0}} \rightarrow \mathbf{0}} \frac{\tilde{\mathbf{A}}\left(\tau_{\mathbf{0}}+\Delta \tau_{\mathbf{0}}\right)-\tilde{\mathbf{A}}\left(\tau_{\mathbf{0}}\right)}{\Delta \tau_{\mathbf{0}}}=\left(\begin{array}{cc}
\mathbf{m}\left(\tau_{\mathbf{0}}\right) & -\mathbf{n}\left(\tau_{\mathbf{0}}\right) \\
\mathbf{n}\left(\tau_{\mathbf{0}}\right) & -\mathbf{m}\left(\tau_{\mathbf{0}}\right)
\end{array}\right)
$$

where

$$
\mathbf{m}\left(\tau_{\mathbf{0}}\right)=\boldsymbol{\alpha}-\frac{\lambda\left(\tau_{\mathbf{0}}\right)}{\mathbf{2}} \boldsymbol{\Gamma}, \quad \mathbf{n}\left(\tau_{\mathbf{0}}\right)=\frac{\lambda\left(\tau_{\mathbf{0}}\right)}{\mathbf{2}} \boldsymbol{\Gamma} .
$$

and $\boldsymbol{\alpha}$ and $\boldsymbol{\Gamma}$ are the discrete analogs of the scattering coefficient profile in the line and the frequency redistribution law, respectively. It is assumed for simplicity that both of these quantities are independent of optical thickness. $\boldsymbol{\alpha}$ is a diagonal matrix with elements $\alpha_{\mathbf{i}}=\alpha\left(\mathbf{x}_{\mathbf{i}}\right)$.

Eq. (149) leads to the system of equations (Nikoghossian, 2013, 2014a]

$$
\begin{gathered}
\frac{d \mathbf{P}}{d \tau_{0}}=\mathbf{m}\left(\tau_{\mathbf{0}}\right) \mathbf{P}\left(\tau_{\mathbf{0}}\right)-\mathbf{n}\left(\tau_{\mathbf{0}}\right) \mathbf{S}\left(\tau_{\mathbf{0}}\right), \\
\frac{d \mathbf{S}}{d \tau_{0}}=\mathbf{n}\left(\tau_{\mathbf{0}}\right) \mathbf{P}\left(\tau_{\mathbf{0}}\right)-\mathbf{m}\left(\tau_{\mathbf{0}}\right) \mathbf{S}\left(\tau_{\mathbf{0}}\right),
\end{gathered}
$$

with initial conditions $\mathbf{P}(0)=\mathbf{I}$ and $\mathbf{S}(0)=\mathbf{0}$, where $\mathbf{0}$ is the zero matrix.

The matrix $\mathbf{P}\left(\tau_{0}\right)$ obtained by solving the system of Eqs. (151), (152) can be inverted to determine the desired values of the reflection and transmission coefficients of the medium. The analogous system for the rest of other properties of the medium reads

$$
\begin{aligned}
\frac{d \mathbf{M}}{d \tau_{0}} & =-\mathbf{m}\left(\tau_{\mathbf{0}}\right) \mathbf{M}\left(\tau_{\mathbf{0}}\right)-\mathbf{n}\left(\tau_{\mathbf{0}}\right) \overline{\mathbf{S}}\left(\tau_{\mathbf{0}}\right), \\
\frac{d \overline{\mathbf{S}}}{d \tau_{0}} & =\mathbf{n}\left(\tau_{\mathbf{0}}\right) \mathbf{M}\left(\tau_{\mathbf{0}}\right)+\mathbf{m}\left(\tau_{\mathbf{0}}\right) \overline{\mathbf{S}}\left(\tau_{\mathbf{0}}\right) .
\end{aligned}
$$

with the initial conditions $\mathbf{M}(0)=\mathbf{I}$ and $\mathbf{S}(0)=\mathbf{0}$.

If the medium is homogeneous, then it is sufficient to limit ourselves to the system of Eqs. (151) and (152), the solution of which can be written in the form of a series expansion of the matrix exponential

$$
\mathbf{P}\left(\tau_{0}\right)=\mathbf{I}+\left(\boldsymbol{\alpha}-\frac{\lambda}{2} \boldsymbol{\Gamma}\right) \frac{\tau_{0}}{\mathbf{1 !}}+\left[\boldsymbol{\alpha}^{2}-\frac{\lambda}{2}(\boldsymbol{\Gamma} \boldsymbol{\alpha}-\boldsymbol{\alpha} \boldsymbol{\Gamma})\right] \frac{\tau_{0}^{2}}{2 !}+\ldots
$$




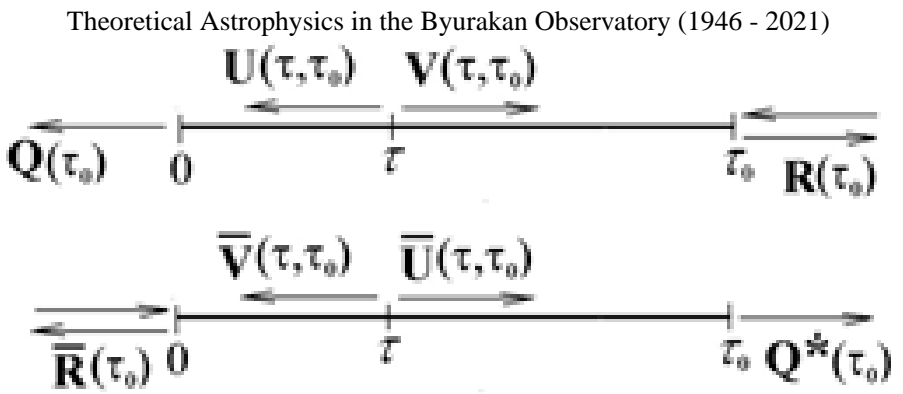

Figure 14: Illustrating the determination of a radiation field inside a medium.

$$
\mathbf{S}\left(\tau_{\mathbf{0}}\right)=\mathbf{R}\left(\tau_{\mathbf{0}}\right) \mathbf{P}\left(\tau_{\mathbf{0}}\right)=\frac{\lambda}{\mathbf{2}} \boldsymbol{\Gamma} \frac{\tau_{\mathbf{0}}}{\mathbf{1} !}+\frac{\lambda}{\mathbf{2}}(\boldsymbol{\Gamma} \boldsymbol{\alpha}-\boldsymbol{\alpha} \boldsymbol{\Gamma}) \frac{\tau_{\mathbf{0}}^{2}}{2 !}+\ldots
$$

Depths translation groups. The group-theoretical description of radiation fields in inhomogeneous atmospheres was developed in (Nikoghossian, 2014b). A definition of the group of optical depth translations was given and demonstrated its equivalence to the group of compositions of atmospheres. The transition from one optical depth to another by attaching a new layer to it is, in fact, an infinite set of translations of this kind and forms a group, since if the group product is taken to be the result of two successive translations, then it will satisfy all the group postulates. In accord with the physical significance of the problem, the values of the optical depth obtained as the result of translations should not exceed the optical thickness of the medium being considered $\left(\tau \leq \tau_{0}\right)$. This group, as a subset of the $\mathrm{GN}(2, \mathrm{C})$ group, is equivalent to the analogous subgroup introduced in the first part of this paper for a composition of media with different optical thicknesses.

The theory was applied, as above, to an inhomogeneous plane-parallel atmosphere of optical thickness $\tau_{0}$ illuminated from the side of the boundary $\tau=\tau_{0}$ (top sketch in Fig. 14). In general, scattering in this medium can be accompanied by redistribution with respect to both frequency and direction. To describe the radiation field inside the atmosphere, it is introduced the matrices $\mathbf{U}\left(\tau, \tau_{\mathbf{0}}\right)$ and $\mathbf{V}\left(\tau, \tau_{\mathbf{0}}\right)$, which specify the probability that a photon with spatial - frequency characteristics $(\eta, x)$ incident on the boundary at $\tau=\tau_{0}$ will, as a result of diffusion, end up at depth $\tau$, moving, respectively, toward the boundaries $\tau=0 . \tau=\tau_{0}$ generally with some other characteristics $\eta^{\prime}, x \prime$.

Given the probabilistic significance of $\mathbf{U}\left(\tau, \tau_{\mathbf{0}}\right)$ and $\mathbf{V}\left(\tau, \tau_{\mathbf{0}}\right)$, one can write

$$
\mathbf{Q}\left(\tau_{\mathbf{0}}\right)=\mathbf{Q}(\tau) \mathbf{U}\left(\tau, \tau_{\mathbf{0}}\right), \quad \mathbf{V}\left(\tau, \tau_{\mathbf{0}}\right)=\mathbf{R}(\tau) \mathbf{U}\left(\tau, \tau_{\mathbf{0}}\right),
$$

which implies that

$$
\mathbf{U}\left(\tau, \tau_{\mathbf{0}}\right)=\mathbf{P}(\tau) \mathbf{Q}\left(\tau_{\mathbf{0}}\right), \quad \mathbf{V}\left(\tau, \tau_{\mathbf{0}}\right)=\mathbf{S}(\tau) \mathbf{Q}\left(\tau_{\mathbf{0}}\right)
$$

It is seen that in a vector-matrix formulation, the variables in $\mathbf{U}\left(\tau, \tau_{\mathbf{0}}\right)$ and $\mathbf{V}\left(\tau, \tau_{\mathbf{0}}\right)$ are separated, which is one of the advantages of the approach we are using.

It follows directly from Eq. (158) that the subgroup of the representations $\mathfrak{J}(g)$ corresponding to the composition of media with different optical thicknesses is, at the same time, the group of representations for translations of optical depths. Note that, in this sense, a group of compositions of media with different thicknesses can be referred to nominally as a group of translations of optical thicknesses. Therefore, the supermatrix $\sim$ A plays a fundamental role, both when combining atmospheres with different optical thicknesses and for optical depths translation of radiation field inside a specified inhomogeneous atmosphere. In other words, it can be regarded simultaneously as the "composer" (former) of an inhomogeneous atmosphere and the "translator" for the transition from one depth of the medium to another.

It is important to note that, as Eq.(158) implies, in the transition from one depth to another only the global optical characteristics of the added layer are important, while the characteristics of the radiation field within the layer play no role in this transformation. Here it does not matter what particular parameter or parameters have changed to produce the inhomogeneity of the medium. Thus, the approach described here is also suitable for problems involving atmospheres that have different kinds of inhomogeneities and have arbitrary distributions.

Eq.(158) and translation rules ((151), (152)) lead to the ordinary transfer equations for the operator functions $\mathbf{U}$ and $\mathbf{V}$,

$$
\frac{d \mathbf{U}}{d \tau}=\mathbf{m}(\tau) \mathbf{U}\left(\tau, \tau_{\mathbf{0}}\right)-\mathbf{n}(\tau) \mathbf{V}\left(\tau, \tau_{\mathbf{0}}\right)
$$




$$
\frac{d \mathbf{V}}{d \tau}=\mathbf{n}(\tau) \mathbf{U}\left(\tau, \tau_{\mathbf{0}}\right)-\mathbf{m}(\tau) \mathbf{V}\left(\tau, \tau_{\mathbf{0}}\right)
$$

with initial conditions $\mathbf{U}\left(\mathbf{0}, \tau_{\mathbf{0}}\right)=\mathbf{Q}\left(\tau_{\mathbf{0}}\right), \mathbf{V}\left(\mathbf{0}, \tau_{\mathbf{0}}\right)=\mathbf{0}$.

It is easy to see that the group of translations of the optical depths in this problem is the same as the subgroup of the group of compositions of media $\operatorname{GNH}(2, \mathrm{C})$ introduced above. This implies that, when determining the radiation field inside a medium, it is possible to proceed without solving any new equations, since it can be found using the results obtained in constructing a given medium by composition of proper layers and multiplying the found value of $\mathbf{Q}\left(\tau_{0}\right)$ by the values of $\mathbf{P}$ and $\mathbf{S}$ obtained in intermediate calculations.

The far reaching analogy between the group $\mathrm{GN}(2, \mathrm{C})$ and the group of translations of optical depths makes it possible to transfer various results for the global optical characteristics of atmospheres to the case of quantities describing the radiation field within them.

Thus, the media composition groups and their representations generalize the layers adding method, which now covers inhomogeneous, particularly multi-component, atmospheres with allowance of the angle and frequency distribution of the radiation field. The group representations being expressed in terms of some combined discrete quantities allow to find the most general summation laws for reflectance and transmittance of the plane-parallel media. This confirms the importance of the approach proposed in (Nikoghossian, 2013b,c), according to which for determination of the radiation field in the medium one must preliminary find its global optical properties. This contrasted with the approach adopted in the classical transfer theory. Usage of composers facilitates solution of a rather broad class of the line-formation problems by reducing the most part of computations to the simple algebraic operations with matrices. The efficacy of the approach becomes more discernable in the case of multi-component atmospheres often encountered in astrophysical applications.

Employment of infinitesimal operators of the introduced groups makes it possible to establish the close connection of the introduced groups with the classical transfer equations and the equations ensuing from invariant imbedding. In fact, the first of them are connected with the depth translation groups, while the second - with composition groups for media of different optical thicknesses.

The developed theory facilitates solution of some other standard transfer problems of astrophysical importance as, for instance, the problem of multiple scattering in inhomogeneous atmosphere with internal energy sources or in a semi-infinite atmosphere, the problem of finding different statistical mean quantities, characteristics of the radiation diffusion and so on. The theory is of sufficiently great generality since it does not depend on the nature of inhomogeneity of the media, as well as on the angle and frequency distribution of the radiation field.

Hamilton's principle. It was shown (Nikoghossian, 2014b) that differential representations of composition and translation groups reduce the solution of the radiation transfer problems to systems of linear differential equations with initial conditions. The first of these for a homogeneous atmosphere is given by Eqs.(151,152), and the second by (Eqs. (159), (160)).

The linear system of Eqs. $(151,152)$ is analogous to non-linear equations obtained by invariant imbedding (Chandrasekhar, 1960; Sobolev, 1963; Bellman \& Wing, 1973). At the same time, (Eqs. (159), (160)) are the classical radiative transfer equations. If the first of these two systems is first solved and the transmission coefficient is known, then the transport problem (Eqs. (159), (160)) can be formulated as a problem with initial conditions. Mathematically, we are dealing with one and the same system of equations with different initial conditions; this is because of the simple relationships coupling the global optical characteristics of the medium and the quantities describing the emission regime inside the medium. Systems of equations of this type can be reduced to a second order matrix differential equation

$$
\Phi^{\prime \prime}=\mathbf{k}^{2} \boldsymbol{\Phi},
$$

with appropriate initial conditions; then $k^{2}=(\alpha-2 n) \alpha$. In the first case, here $\Phi$ signifies $\mathbf{P}+\mathbf{S}$ and, in the second, $\mathbf{U}$ $+\mathbf{V}$. The operator acting in Eq. (161) is self-adjoint (potential), so a variational principle can be applied to this type of problem (Vainberg, 1964). This possibility is important for the theory of radiative transfer. A variational formulation of these problems of continuum physics is the shortest way to clarify the general physical laws of the phenomena being studied and interpret them. Generalized expressions for the Lagrangian density and the Euler-Lagrange equation can be written in this case for the matrix-vector operator in Eq. (161) (Nikoghossian, 2014a):--

$$
\begin{gathered}
L\left(\boldsymbol{\Phi}, \boldsymbol{\Phi}^{\prime}, \mathbf{k}, \tau_{\mathbf{0}}\right)=\boldsymbol{\Phi}^{2}+\mathbf{k}^{-2} \boldsymbol{\Phi}^{\prime 2}, \\
\frac{d}{d \tau} \frac{\partial L}{\partial \boldsymbol{\Phi}^{\prime}}-\frac{\partial L}{\partial \boldsymbol{\Phi}}=0 .
\end{gathered}
$$


For the two problems being discussed here, the Hamilton's principle (by analogy with the principle of the least action in analytic mechanics) is written in the form

$$
\delta \int \Lambda\left(\Phi, \boldsymbol{\Phi}^{\prime}, \mathbf{k}, \tau_{\mathbf{0}}\right) d \tau_{0}=0, \quad \delta \int \Lambda\left(\boldsymbol{\Phi}, \boldsymbol{\Phi}^{\prime}, \mathbf{k}, \tau\right) d \tau=0
$$

where $\Lambda$ is the Lagrangian.

It is easy to see that these generalized formulas have a significance analogous to that in the scalar problem. Mathematically, this general approach is extremely important, since it covers an extremely wide circle of physical problems. One example is the diffuse reflection and transmission of a three-dimensional medium with isotropic scattering considered in Sect. 5.xi (Nikoghossian, 1999).

Conservation laws. Quadratic and bilinear integrals. The characteristic feature of the matrix equation Eq. (161) is that the optical thickness of the medium and the optical depth do not appear explicitly in it. In other words, these quantities are cyclic coordinates, which leads to conservation laws expressed as constancy of the Hamiltonian, i.e.,

$$
\sum\left(L-\frac{\partial L}{\partial \boldsymbol{\Phi}^{\prime}} \boldsymbol{\Phi}^{\prime}\right)=\text { const }
$$

or

$$
\sum\left(\Phi^{2}-\mathbf{k}^{-2} \Phi^{\prime 2}\right)=\text { const. }
$$

For the above three-dimensional problem of isotropic scattering, Eq. (166) takes the form

$$
\int_{0}^{1}\left[\Phi^{2}-\left(\eta \Phi^{\prime}\right)^{2}-\lambda \Phi J\right] d \eta=\text { const. }
$$

In considering the internal radiation regime, we have $\Phi=U+V$, and $\eta \Phi^{\prime}=U-V$, so that Eq. (161) yields

$$
\int_{0}^{1} U\left(\tau, \eta, \mu ; \tau_{0}\right) V\left(\tau, \eta, \mu ; \tau_{0}\right) d \eta-\frac{\lambda}{4}\left(\int_{0}^{1}\left(U\left(\tau, \eta, \mu ; \tau_{0}\right)+V\left(\tau, \eta, \mu ; \tau_{0}\right)\right) d \eta\right)^{2}=\text { const. }
$$

This equation is the so-called $Q$ integral of Rybicky (1977) written in our notation. The constant of integration is found from the boundary conditions: it equals

$$
\text { const }=-\frac{\lambda}{4}\left(\int_{0}^{1} Q\left(\eta, \mu, \tau_{0}\right) d \eta\right)^{2}
$$

for a finite medium and zero for an infinite medium. This dependence can be dealt with most easily in a physical sense if we examine the scalar analog of the problem. Then Eq. (168) yields

$$
H^{2}(\tau)-(1-\lambda) \Phi^{2}(\tau)=\lambda Q^{2}\left(\tau_{0}\right),
$$

where $H=U-V$ and $\Phi=U+V$. These quantities are related to the radiative flux and the integrated intensity (or source function), respectively. It shows that in a medium with a given optical thickness and a specified scattering coefficient at its every depth, a mutually unique relation develops between the integrated radiation field and its flux; i.e., when more radiative energy is confined in the medium, its flux is greater and vice versa. In particular, in the two limiting cases of $\lambda=0$ and $\lambda=1$, we have $H(\tau)=U(\tau)$ and $H(\tau) \equiv Q\left(\tau_{0}\right)$. Finally, in a semi-infinite atmosphere we have $H(\tau)=\sqrt{1-\lambda} \Phi(\tau)$. It should be specially noted that in this problem it is possible to derive a number of other integrals, in particular the $R$-integral. These integrals, however, are consequences of a conservation law and have no independent significance (Nikoghossian, 1999). In similar fashion, the Hamilton principle is applied for the global optical characteristics of a scattering atmosphere, when the cyclic coordinate in Eqs. $(151,152)$ is $\tau_{0}$ according to the Lagrangian. In this case, the system of matrix equations (151) and (161) leads to the conservation law

$$
\int_{0}^{1} P\left(\tau_{0}, \eta, \mu\right) S\left(\tau_{0}, \eta, \mu\right) d \eta-\frac{\lambda}{4}\left(\int_{0}^{1}\left[P\left(\tau_{0}, \eta, \mu\right)+S\left(\tau_{0}, \eta, \mu\right)\right] d \eta\right)^{2}=-\frac{\lambda}{4} .
$$

For a one-dimensional medium it takes the form

$$
\lambda(1+R)^{2}-4 R=\lambda Q^{2} .
$$

This integral is well known in the classical theory of radiative transfer (Ambartsumian, 1960; Sobolev, 1963). It has a simple physical significance that indicates the relationship between the reflection and transmission capacities of a given atmosphere is fully determined by the magnitude of the scattering coefficient. The results shown in this 
section answer the questions posed in (Rybicky, 1977) regarding the nature of the quadratic integrals of the transfer theory. The group approach also makes it possible to extend the results obtained there, as well as in (Ivanov, 1978; Janovitskij, 1997), to the quantities which determine the reflectivity and transmission of a scattering and absorbing atmosphere. The additivity of the linear operator in Eq. (161) shows possibility to derive so-called bilinear integrals, which connect with each other the solutions of different radiative transfer problems.

Multiparameter groups. The definition of the composition group for absorbing and scattering media given above is sufficiently general, since it imposes no limits on the physical and optical properties of the combined media. They can be inhomogeneous and have different optical thicknesses. Compositions of homogeneous media that differ from one another in several parameters characterizing the diffusion process in them are of special interest. Depending on the number of these parameters, the resulting groups are one-, two-, or three-parameter groups, etc. In the limiting transition to infinitesimal thicknesses, multicomponent groups usually lead to a linear partial differential equation of order determined by the number of parameters that vary independently of one another.

As examples, we point at two problems of the theory of radiative transfer which are reduced to solving equations of one and the same kind. The first is the nonstationary radiative transfer problem in a homogeneous medium of finite optical thickness. We shall be interested in the dependence of the reflection and transmission coefficients on the thickness of the medium and time under the assumption that as they diffuse, the photons spend time only in flight between two successive scattering events. The average time of flight $T$ is determined by the density $n$ of the medium and the average value of the absorption coefficient in the line $k: T=\left(c k n^{-1}\right.$. In the following we assume that the time $\omega$ is measured in units of $T$. The addition law for these media will again be given by Eq. (159), where the indices $i$ ( $i=1,2,1 \mathrm{U} 2$ ) now belong both to the optical thickness $\tau_{0}$ of the given medium and to the dimensionless time $\omega$. For simplicity we consider the problem in a one-dimensional approximation assuming that scattering in the medium takes place with complete frequency redistribution when $\Gamma=\alpha_{0} \alpha_{0}^{\prime}$, with the elements of the diagonal matrices $\alpha_{0} \alpha_{0}^{\prime}$ being the values of the absorption coefficient in a spectrum line normalized in corresponding fashion. The addition law for this problem reduces to the following hyperbolic system of the linear partial differential equations of the first-order with two independent variables:

$$
\frac{\partial \mathbf{P}}{\partial \tau_{0}}+\frac{\partial \mathbf{P}}{\partial \omega}=\mathbf{m} \mathbf{P}\left(\omega, \tau_{\mathbf{0}}\right)-\mathbf{n S}\left(\omega, \tau_{\mathbf{0}}\right), \quad \frac{\partial \mathbf{S}}{\partial \tau_{\mathbf{0}}}+\frac{\partial \mathbf{S}}{\partial \omega}=\mathbf{n} \mathbf{P}\left(\omega, \tau_{\mathbf{0}}\right)-\mathbf{m} \mathbf{S}\left(\omega, \tau_{\mathbf{0}}\right),
$$

with the conditions $\mathbf{P}(\omega, \mathbf{0})=\mathbf{I}, \mathbf{S}(\omega, \mathbf{0})=\mathbf{0} ; \mathbf{P}\left(\mathbf{0}, \tau_{\mathbf{0}}\right), \mathbf{S}\left(\mathbf{0}, \tau_{\mathbf{0}}\right)$. Treating the left side of Eq. (173) as the derivative with respect to direction and making the corresponding parametrization, the unknown functions can be represented as functions of a single variable which vary in a direction perpendicular to the characteristic direction (Arsenin, 1984). Along this direction the equations have the same form as the equations discussed above. In particular, by analogy with Eq. (161) we have

$$
\widetilde{\Phi}^{\prime \prime}{ }_{s}=\mathbf{k}^{2} \breve{\Phi}(\mathbf{s}),
$$

where $\breve{\Phi}(s)=\breve{\mathbf{P}}(s)+\breve{\mathbf{S}}(s)$ and $\mathbf{k}^{\mathbf{2}}=\mathbf{m}^{\mathbf{2}}-\mathbf{n}^{\mathbf{2}}$.

Using the variational principle along this direction makes it possible to introduce the Lagrangian density,

$$
L=\left(\frac{\partial}{\partial \tau_{0}}+\frac{\partial}{\partial \omega}\right)\left[\mathbf{P}^{2}+\mathbf{S}^{2}-\frac{\lambda}{2}(\mathbf{P}+\mathbf{S})^{2}\right]
$$

for this problem along with a conservation law in the form

$$
\sum\left(\frac{\partial}{\partial \tau_{0}}+\frac{\partial}{\partial \omega}\right)\left[\frac{\lambda}{4} \boldsymbol{\alpha}_{0}(\mathbf{P}+\mathbf{S})^{2}-\mathbf{P S}\right]=0
$$

where the arguments of the unknown matrix-functions in Eqs. (175) and (176) have been left out for brevity. The sum in Eq. (176) is taken over one of the columns denoting the frequency of the reflected radiation. In the stationary problem the time dependence vanishes and we find

$$
\sum \frac{\lambda}{4} \alpha_{0}(\mathbf{P}+\mathbf{S})^{2}-\mathbf{P S}=\text { const. }
$$

The second problem involves determining the profiles of spectrum lines formed in a homogeneous scattering and absorbing atmosphere of finite thickness with developed homogeneous turbulence. Referring the reader to (Nikoghossian, 2017) for a statement of the problem and its physical aspect, here we note only that an approach based on the group properties of the quantities describing radiative transfer can be used in this problem as well if the spatial correlations of non-thermal motions are represented as a Markov process, which is described by Gaussian distributions in a plane. For this distribution in this case, the Chapman-Kolmogorov relation applies, i.e., 


$$
G\left(u, u^{\prime}, \rho\left(l_{1}+l_{2}\right)\right)=\int_{-\infty}^{\infty} G\left(u, u^{\prime \prime}, \rho\left(l_{1}\right)\right) G\left(u, u^{\prime \prime}, \rho\left(l_{2}\right)\right) d u^{\prime \prime} .
$$

The distribution $G$ has a probabilistic sense in that $G\left(u, u^{\prime}, \rho(l)\right) d u$ is the probability that if at some depth $\tau^{\prime}$ the nonthermal velocity equals $u^{\prime}$, then at depth $\tau$ it takes a value within the interval $(u, u+d u)$. The correlation coefficient $\rho$ for Markov process varies exponentially as $\rho(l)=\exp (-l / \Lambda)$, where $\Lambda$ is the average optical correlation length. Then the addition law for two layers with optical thicknesses $\tau_{1}$ and $\tau_{2}$ is given by

$$
\left(\begin{array}{l}
\mathbf{P}_{\mathbf{1} \cup \mathbf{2}}(\mathbf{u}) \\
\mathbf{S}_{\mathbf{1} \cup \mathbf{2}}(\mathbf{u})
\end{array}\right)=\left(\begin{array}{ll}
\mathbf{P}_{\mathbf{2}}\left(u^{\prime}\right) & -\overline{\mathbf{S}}_{\mathbf{2}}\left(u^{\prime}\right) \\
\mathbf{S}_{\mathbf{2}}\left(u^{\prime}\right) & \mathbf{M}_{\mathbf{2}}\left(\mathbf{u}^{\prime}\right)
\end{array}\right) G\left(\rho\left(\tau_{1}\right)\right)\left(\begin{array}{c}
\mathbf{P}_{\mathbf{1}}(u) \\
\mathbf{S}_{\mathbf{1}}(u)
\end{array}\right) .
$$

Here the variable $u$ denotes the shift of the frequency at the right boundaries of these media. The system of vectormatrix differential equations generated by this rule is given by

$$
\begin{gathered}
\frac{\partial \mathbf{P}}{\partial \tau_{0}}+\frac{1}{\Lambda} \frac{\partial \mathbf{P}}{\partial u}=\mathbf{m}(u) \mathbf{P}\left(u, \tau_{\mathbf{0}}\right)-\mathbf{n}(u) \mathbf{S}\left(u, \tau_{\mathbf{0}}\right), \\
\frac{\partial \mathbf{S}}{\partial \tau_{0}}+\frac{1}{\Lambda} \frac{\partial \mathbf{S}}{\partial u}=\mathbf{n}(\mathbf{u}) \mathbf{P}\left(\mathbf{u}, \tau_{\mathbf{0}}\right)-\mathbf{m}(\mathbf{u}) \mathbf{S}\left(\mathbf{u}, \tau_{\mathbf{0}}\right),
\end{gathered}
$$

with the initial conditions $\mathbf{P}(u, 0)=\mathbf{I}$ and $\mathbf{S}(u, 0)=\mathbf{0}$. $\mathbf{m}$ and $\mathbf{n}$ are specified by the same formulas as in the preceding example with the frequency shift taken into account. The system of Eqs. (180) is valid for any values of the correlation coefficient except $\Lambda=0$. This case, which corresponds to a microturbulent regime is generally examined separately (Nikoghossian, 2017). We can see that this system of equations differs in form from Eqs. (173) derived for the preceding problem in that the correlation coefficient $\Lambda$ appears on its right hand side. Thus, both the methods for studying and solving it, as well as the possibility of using the variational principle with conservation laws, are fundamentally the same.

\section{APLICATIONS TO ASTROPHYSICAL PROBLEMS. INTERPRETATION OF SPACE OBJECTS SPECTRA.}

\section{THEORETICAL PHYSICS}

i. The relativistic Doppler broadening of the line absorption profile. The classical results of Doppler broadening of the spectral line absorption profile was generalized in (Kichenassami, Krikorian \& Nikoghossian, 1982) in a relativistic gas in thermal equilibrium by taking into account the relativistic variance of the volume absorption coefficient of the gas, as derived in (Thomas, 1930). The latter was shown to be important, since the variance produces a small correction even in the non-relativistic approximation. The derived expression of the absorption coefficient $\chi_{v}$, has a form

$$
\chi_{v}=\frac{n_{0}}{2} \frac{\sigma_{0} f}{K_{2}(m \xi)} \frac{\Gamma}{\pi v^{2}} \int_{-\infty}^{\infty} \mathrm{e}^{-\frac{m \xi\left(x^{2}+v^{2}\right)}{2 v x}} \frac{x d x}{(x-v)^{2}+\Gamma^{2}},
$$

where the following notations are used: $n_{0}$ is density of the gas in its rest frame, $\sigma_{0}=\pi e^{2} / m_{e} c, \xi=c^{2} / k T, c$ is light velocity, $x=\gamma v(1+\beta), \gamma=\left[1+(p / m)^{2}\right]^{1 / 2}$. The modified Bessel function of the second order $K_{2}(m \xi)$ is defined by

$$
K_{2}(m \xi)=2 \int_{0}^{\infty} \mathrm{e}^{-m \xi c h t} c h t d t .
$$

For vanishing $\Gamma$ Eq. (182) gives

$$
\chi_{v}=n_{0} \sigma_{0} f \frac{v_{0}}{2 K_{2}(m \xi) v^{2}} \mathrm{e}^{-\frac{m \xi\left(v^{2}+v_{0}^{2}\right)}{2 v v_{0}}},
$$

where $v_{0}$ is the frequency in the center of the line.

ii. The photon scattering by electrons. Energy losses by electrons. A series of papers in Byurakan Observatory were devoted to derivation of the redistribution function (scattering kernel) describing the photons scattering on free electrons. The simplest special case of isotropic and low energetic photons scattering on the ensemble of electrons with the Maxwellian distribution of moments was considered above in Sect. 5.i (Harutyunian \& Nikoghossian, 1980). The generalized problem for the redistribution law averaged over directions of monoenergetic electrons velocities in the observer's frame was treated in (Harutyunian \& Nikoghossian, 1981, 1989; Dzhrbashian \& Harutyunian, 1985). The explicit expression of this law is too cumbersome to be presented here. We limit ourselves with only mentioning that the first of the cited two papers uses the obtained results to formulate the problem of diffuse reflection of radiation from a semi-infinite absorbing and electron scattering atmosphere. Exact expressions for the energy losses during photons scattering is treated and investigated in (Harutyunian, Dzhrbashian \& Nikoghossian, 1988).

The results of numerical calculations for the energy loss by electrons and photons depending on their energy are depicted in Fig.15. It is noteworthy the maxima of curves for the electrons energy loss. 

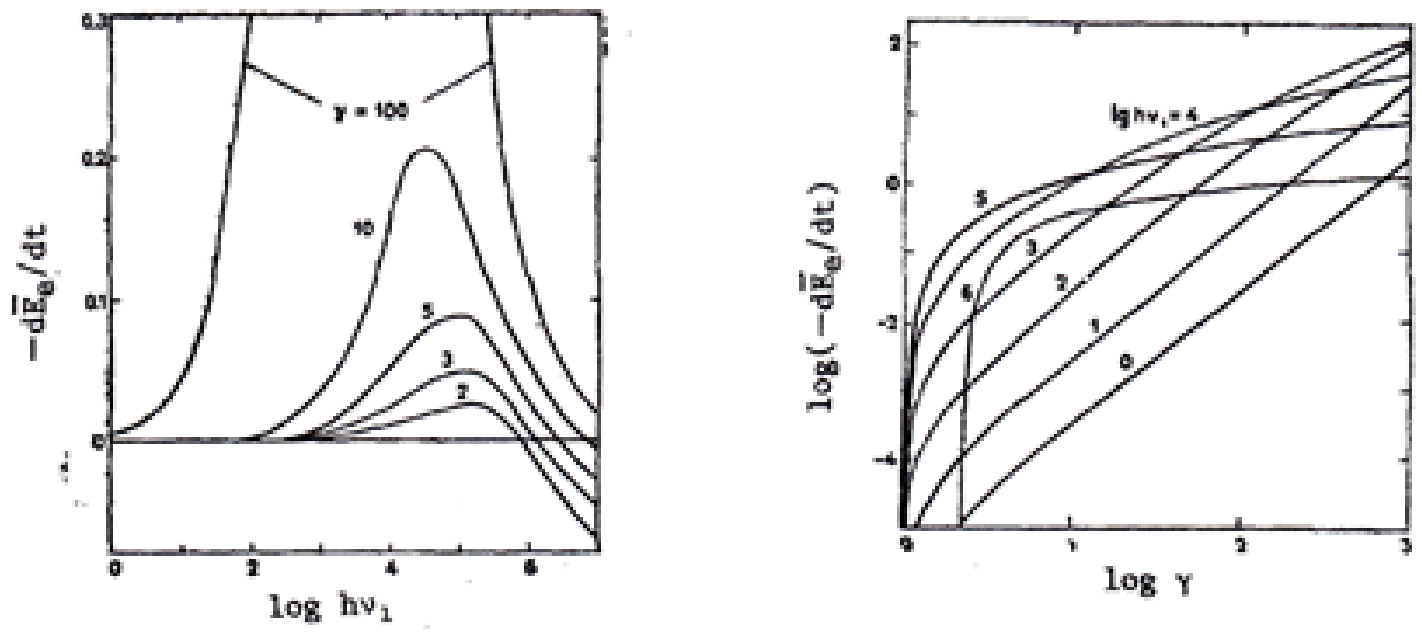

Figure 15: The angle-averaged value of the energy loss by electrons as a function of the energy of photons and electrons (left panel); the dependence of the energy loss by photons on the energy of the photons and electrons (right panel).

\section{SOLAR PHYSICS}

\subsection{Quiescent prominences.}

i. The research work on solar prominences carried out in collaboration with the Meudon observatory in Paris started in 1994. The cooperation involved Z. Mouradian (Meudon), A. Nikoghossian (Byurakan observatory) and their PhD student S. Pojoga (Romania). The first works used digital raster images of prominences obtained with the Harvard College Observatory (HCO) aboard ATM-Skylab, which represent a rich observational resource for investigating the statistical properties of the spectra formation in media with composite thread-like structure. Spatial and temporal variations of the prominences brightness in the EUV spectral domain were studied. The spatial brightness variations of prominences may have several reasons: differences in the line-of-sight number of structural elements and in the value of the filling factor, physical inhomogeneities of the structures, instrumental errors and so on. These factors are studied separately in order to determine the significance of each in establishing the variations.

ii. The theory developed in a series of papers by Nikoghossian, Mouradian \& Pohoga (1997, 1998a, 1998b, 1999), Pojoga, Nikoghossian \& Mouradian (1998), Nikoghossian \& Mouradian (2000), Nikoghossian (2001), Nikoghossian, Aboudarham \& Mouradian (2005) aimed at determining the mean intensity and the relative mean square deviation (RelMSD) for the line radiation emerging from an one-dimensional atmosphere with randomly distributed inhomogeneities. The idea of such statistical approach based on the study of spatial fluctuations of brightness goes back to Ambartsumian's classical works $(1951,1960)$ studying fluctuations of the Milky Way brightness in estimating the mean value of the opacity of interstellar matter.

iii. The theory developed in the cited papers both for spectral lines in the LTE and Non-LTE, allowed to make an idea on the processes leading at the observed spatial and temporal fluctuations of brightness, and then on the optical thicknesses, temperature and velocity field. Some information on the line-of-sight number of threads was obtained by theoretical treatment of dependence of the filling factor (coefficient of filling of the spectrograph slit in each pixel) on this number (Nikoghossian, 2001). It was shown that the role of the random variations in the filling factor is insignificant among the other factors producing brightness fluctuations for the regions of prominences having high and moderate packing of filaments. At the same time, this role may become important for rarified and weakly radiating regions. Note that analogous approach is applicable also in studying such multicomponent and non-steady formations in the solar atmosphere as chromospheric spicules and various coronal streams. Similar examples can also be cited from other fields of astrophysics.

iv. The classical method for determining the velocities of micro-turbulent motions in solar prominences (TandbergHanssen, 1995) was generalized in (Nikoghossian \& Mouradian 2010) to account for the possible opacity of the spectral lines. A new characteristic of a line was introduced, which, for a given line formation mechanism, allows also to determine the optical thickness of the emitting region. The method was applied to the spectral lines in the EUV region observed with the SUMER spectrograph as part of the SOHO space program. Comparison with observational data not only confirms the validity of this mechanism for the line formation, but also shows that the optical thickness of the medium is typically small for these lines. Difficulties involved in determining the kinetic temperature and, therefore, the micro-turbulent velocities, are mainly due to accuracy of the observational material. Based on the lines 


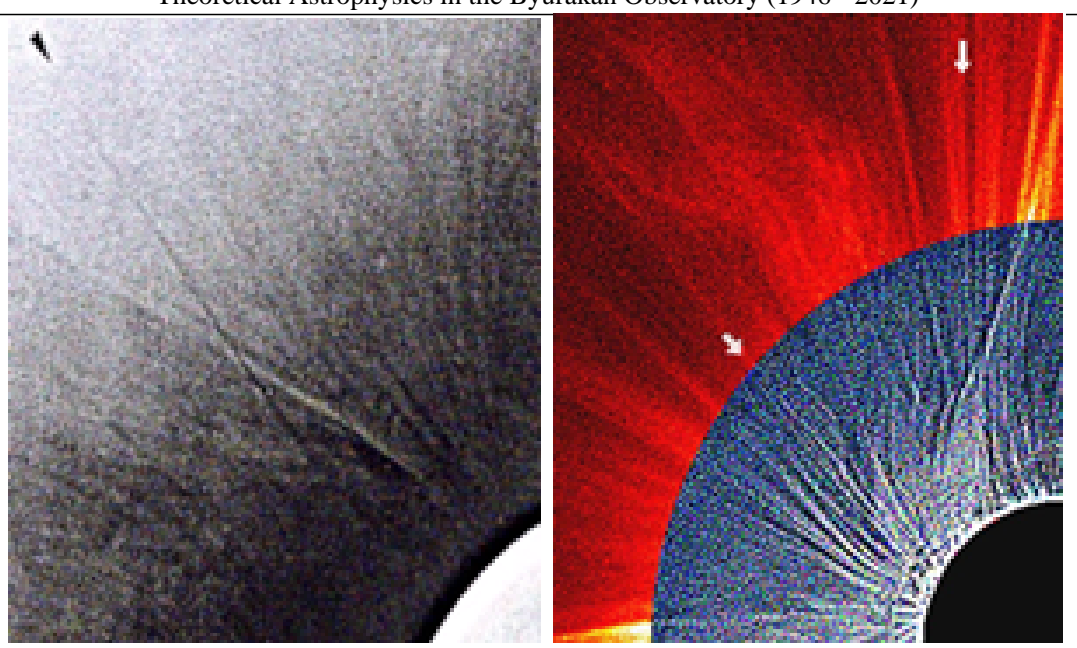

Figure 16: Early observation of a coronal linear (in dark) ray-thread showing a large aspect ratio. This is a greatly magnified negative print made by the team of the Kiev University (Ukraine) using a $10 \mathrm{~cm}$ diameter lens of 10 meters focal length and $30 \mathrm{~s}$ exposure time (left panel). Selected part of a composite (inner part is a W-L compressed image taken from Iran by the team of the Institut d'Astrophysique de Paris-CNRS using a radial filter; outer parts is the Lasco C2 image from SOHO taken simultaneously from space on July 11, 1999) to show the NE limb, right above a coming flaring active regions. High resolution images of the more inner parts of this limb did show many loops. Arrows are indicating the most obvious linear rays-threads. The one raising above the $\mathrm{N}$-pole region was seen on both ground-based and space-born images (right panel).

of various ions, this velocity is estimated to be on the order of $30-40 \mathrm{~km} / \mathrm{s}$. The cited paper presents a table of 20 spectral lines with the proper estimating values of the micro-turbulent velocity, opacity and kinetic temperature.

\subsection{Coronal supra-thermal streams.}

For a long time high resolution W-L eclipse images taken near the time of the solar maximum of activity did show multiple thread-like streams and/or straight and fine "diverging" rays of unknown origin. This structure is not anchored at the surface of the Sun but appears as a straight linear structure starting from a fraction of the solar radius and was sometimes called rays or "fan-streamer" (Vsessviatski \& Bougoslavskaya 1944; Koutchmy 1969). They are typically seen above limb active regions which produced flares or at least sub-flares before. Coronal streams are one of the intriguing short-lived phenomena emerging from the magnetically dominated part of the inner atmosphere of the Sun; their typical linear structure, their large aspect ratio, and their dispersed directions with respect to the local radial direction strongly suggest that they largely ignore the outer background of the general coronal magnetic field which is usually computed using current-free or force-free extrapolations of the measured magnetic field at the solar surface. They probably represent fluxes of supra-thermal electrons (with velocities up to $10^{5} \mathrm{~km} / \mathrm{s}^{-1}$ ) moving partly along spiral trajectories.

The theoretical works by Nikoghossian \& Koutchmy $(2001,2002)$ aimed at revealing the role of the Compton effect when considering the W-L scattering of the solar photospheric radiation by coronal supra-thermal streams. The height-dependent model problem of Compton scattering on the beam of fast electrons gyrating around the lines of force of the magnetic field was considered. The resulting change in frequency averaged over the beam and the solid angle, within which the photospheric radiation falls, is computed for both sunward and anti-sunward directed streams. The dependence of the effect on the height above the solar surface, the speed of electrons and the slope angle were discussed. For illustration, it was assumed that the frequency distribution of the incident radiation is Planckian. The effective value of the cross-section of interaction was compared with that for the Thomson scattering.

The results may be summarized as follows. At the least, at high distances over the solar limb the frequency change in the suprathermal stream radiation resulting from the Compton scattering may be significant even for moderate energies of electrons. For electrons moving off the Sun, longwave-ward drifts of the spectrum are more likely, while opposite ones are due preferably to the opposite directed beams of electrons. The effect was expected to be detectable only if the fractional density of fast electrons is not very small.

The realistic height-dependent model problem was treated in (Nikoghossian \& Koutchmy, 2002). The results of numerical calculations for the mean frequency change and the proper cross-section for both the sunward and antisunward directed beams of electrons were given as a function of height and the slope angle. It was concluded that, depending on the angle between directions of the initial outburst and magnetic field, the scattering on the beams moving away the Sun may produce measurable drifts in frequency to shortwave as well as to longwave domains of 
the spectrum. At the same time, the sunward directed beams result only in an increase in the photon energy.

In order to verify the theoretical results obtained, observations were made during the total eclipse in Angola in 2001. The authors first document several examples of well observed cases of the linear W-L coronal threads extending above flaring active regions which are good candidates to give the signature known from the interpretation of radio type III bursts. There were performed measurements of the color index of the coronal supra-thermal streams. Observations imply that the color index of streams differs markedly from that for adjacent parts of the surrounding corona taken as reference (Koutchmy \& Nikoghossian, 2005). The registered drifts to the longwave and shortwave domains of the spectrum are in agreement with theoretical results obtained by the authors.

\section{STELLAR ASTROPHYSICS}

8.1. Flare stars. A certain place in the works of the Department of Theoretical Astrophysics occupied the study of flare stars as a part of observational programs in the Byurakan Observatory

i. In the frameworks of this research field, a statistical analysis of the flare data obtained in 1967-1969 for the star UV Cet and in 1967-1970 of the star YZ CMi was carried out by Oskanian \& Terebizh (1971a,b). They showed that the flare activity of these stars did not change during the mentioned periods. The detailed investigation of a number of flares showed that the succession of the flares on the stars are near the Poisson distribution law. The research work was continued by B-color photometric observations of the stars AD Leo, EV Lac, YZ CMi, UV Cet, The distribution of amplitudes, amounts of radiated energy and frequencies were studied. The influence of the observational selection on the detection of small-amplitudes flares as well as the problem of determination of the absolute flare activity of these stars were discussed in detail.

ii. The Ambartsumian paper appeared in 1978 is devoted to derivation of the frequency function of stellar flares in clusters. First, the problem of determining the total number of flare stars was considered on the example of Pleiades under assumption that all stars flare equally often. Assuming that the flares occur according to Poisson's law, the problem was solved by using the chronology of discoveries ("first flares") and the chronology of confirmation ('second flares'). For the expected number of stars in the aggregate that have not flashed in the entire time they were observed $n_{0}$, an estimate was obtained

$$
n_{0}=\frac{n_{1}^{2}}{2 n_{2}}
$$

where $n_{1}$ and $n_{2}$ are expected numbers of stars that respectively flared once and twice.

If frequencies of flares are different for different stars, then this formula gives only the lower limit of this value. It has been shown that in the case of different frequencies, there are the following inequalities

$$
\frac{n_{1}^{2}}{n_{2}} \geq n_{0} \geq \frac{n_{1}^{2}}{2 n_{2}} \text {. }
$$

Further, as subtracted, the fraction of flare stars increases when getting to fainter stars, in other words, the flare activity previously decreases in more luminous stars.

iii. The possibility of interpretation of flare radiation of red dwarf stars with use of the inverse Compton scattering was discussed by Harutyunian, Krikorian \& Nikoghossian (1979). They refined the expressions, adopted earlier for the law of the frequency redistribution during the scattering process and gave the formula for differential cross-section. It was indicated the importance of the geometry of the photon-electron interaction and showed that the interpretation of observational data by this mechanism meets considerable difficulties.

iv. Chavushian, Pikichyan et al. (2004) constructed the three-dimensional distribution of flare stars in the Pleiades cluster. For this purpose a one-dimensional distribution was first constructed from the observed two-dimensional distribution of the stars. It was shown that reliable construction of one-dimensional distribution requires solution of the Abel equation. The last one was used to determine the dependence of the real three-dimensional distribution of the density of flare stars on distance from the center of the cluster. This reveals a spherical layer of width $0.5 \mathrm{pc}$ $2.8 \leq R \leq 3.5 \mathrm{pc}$ with a deficit in the member of flare stars. A profile of the three-dimensional density distribution of flare stars was constructed in the region of deficit. The characteristics of this region were described.

v. The works by Hayrapetian \& Nikoghossian (1989), Hayrapetian, Vikhrev \& Nikoghossian (1989) showed that qualitative and quantitative comparison of the results of plasma experiments with observational data on stellar flares suggests that flares could be explained by the Pinch effect, i.e., by the plasma compression by magnetic field in the outer layers of these stars. A possible scenario of magnetic tubes floating from the convective zone with further stretching and arch structures formation was suggested.

vi. Hayrapetian \& Nikogohossian (1988) showed that some spectral peculiarities of T Tau stars such as the values of half-widths of Balmer lines, the short-term variability of hydrogen lines, the values of Balmer jumps and shifts of the HeI lines, can be explained by generation of the quasi-stationary plasma turbulence in atmospheres of these stars. 
vii. An inverse problem of determination of the total number, luminosity function and the variability characteristics of brightness of T Tau type stars in stellar aggregates was considered by Pikichyan (2018). It was demonstrated that the four observations of stellar aggregate make it possible to estimate the total number of irregular variable stars. By assuming that the random process is continuous and stationary, it was formulated an inverse problem for restoration of the star's luminosity function, as well as the distribution function of the brightness variability. The original information requires detailed photometry of two rather distant in time "pictures" of the aggregate stars. It is significant that the mentioned author with his co-author proposed a project aim at minimizing the effect of the astronomical dome and tower on the quality of the star image (Muradyan \& Pikichyan, 1986). The new class of astronomical domes of changing geometry was suggested, which are smaller in size and simpler in constructing as compared to the classical ones with constant geometry.

\subsection{LBV stars.}

i. The spectral variability of P Cyg was investigated by Israelian \& Nikoghossian (1993). The careful analysis of the CCD - spectra of P Cyg made it possible to identify 10 of the 43 lines that had been unidentified up to that time. The majority of identified lines belong to multiply ionized atoms. The conclusion was reached that there is anomalous heating in the certain layers of the stellar wind. There were presented facts that suggest variation in the degree of ionization of the stellar wind. Some forbidden lines were identified in the spectrum of P Cyg (see, also Israelian \& De Groot, 1992).

ii. Based on the fact that the stellar winds of LBV stars are due to acceleration of matter resulting from radiation pressure in optically thin weak emission lines of metals in the Balmer continuum, Israelian, Nikoghossian \& Tambovtseva (1993) constructed models of the winds of these stars for some specific cases. The obtained results are in satisfactory agreement with observational data.

iii. The papers by Nikoghossian \& Israelian (1996), Israelian \& Nikoghossian (1996) and Nikoghossian (2020) present a theoretical study of changes in some spectral characteristics of the early type supergiants due to the scattering of radiation in the continuous spectrum. Thomson scattering on free electrons was regarded as a scattering mechanism, although the approach used is fairly general. The cause of changes in the star's spectral class at constant bolometric luminosity and surface temperature was revealed, and conditions necessary for their implementation were given. The effect of scattering in the continuum on the magnitude of intensity jumps in the hydrogen spectrum depending on the degree of ionization was studied. The conditions under which the Schuster mechanism of emission lines formation starts to operate both in the isothermal atmosphere and in the atmosphere with temperature gradient were brought out. iv. Possible reasons for displacement of the peak of an emission line of an object with an expanding shell were considered by Grigorian \& Arshakian (1990). A new effect, a blue shift of the emission line peak, was established. It was shown that such peak arises because the simultaneously observed photons reached the observer from points of the shell at different distances emitted at different times of its expansion. The effect is more pronounced, the closer the speed of the ejected layers of the shell to the speed of light. The case of continuous shell isotropically expanding with velocities proportional to the distance from the star was treated. A method was proposed for determining the displacement of the emission peak and estimating the lower limit of the density of atoms emitted a given line. Application of this method to the supernova SN 1987A led to an estimate of the radial velocity $(-170 \mathrm{~km} / \mathrm{s})$ corresponding to the lower limit of the displacement of the peak of the $H_{\alpha}$ line.

\subsection{Ap stars.}

In the context of the study of elemental diffusion in Ap stars, Alecian (Meudon Observatory in Paris) in cooperation with Harutyunian (2012), Haruthunian et al., (2016) treated the problem of radiative transfer in an inhomogeneous medium with the depth-dependent absorption coefficient. The corresponding equations are obtained to solve by numerical methods.

\subsection{Pulsars.}

The radio luminosities of pulsars were given by Andreasian \& Arshakian (1993) as a function of their period and the time of its variation. The parameters of that dependence were calculated and independent distances were determined for 288 pulsars. The average electron densities toward the pulsars were determined from the known dispersion measures. The results obtained were used to study the large-scale electron density distribution in the Galaxy at a distance of 4-5 kps from the Sun. The distribution maximum has been found to lay at a distance of $9 \mathrm{kps}$ from the center of the Galaxy in the vicinity of the Sagittarius spiral arm. They found that the electron density falls off exponentially in the regions between arms (toward the Perseus and Centaurus spiral arms).

\section{EXTRAGALACTIC ASTROPHYSICS}

i. The possibility of explaining the broad emission lines formation in quasars spectra by Cherenkov radiation was considered by Nikoghossian (1994). This idea was suggested and worked out by some authors in a number of papers, where, however, some effects, being essential for the line profile, were ignored. The classical Non-LTE line-formation 
problem with allowance for the Cherenkov radiation was solved in the above-cited paper. The results obtained for the Lyman alpha line for the electron temperature $T_{e} \approx 1.5 \cdot 10^{4} \mathrm{~K}$ show that the effect of this mechanism becomes measurable merely for unreasonably high densities of relativistic electrons $n_{e} \geq 10^{11} \mathrm{~cm}^{-3}$. ii. The statistics of interacting galaxies was treated by Karachentsev \& Terebizh (1968). They used data for the systems of interacting galaxies brighter than $15^{m} .0$ spatial distribution. The results of statistical analysis have proved that the processes giving rise to interacting galaxies have been spontaneous and induced by internal causes.

iii. The source-count analysis and the $V / V_{m}$ test in the study of the evolution of quasi-stellar radio sources was treated by Terebizh (1973). The exact expressions for $V / V_{m}$ the distribution under arbitrary character of their evolution were derived. The dependence of different methods were quantitatively was estimated. Arguments were rendered in favor of the density evolution of quasi-stellar radio sources on the analysis of distribution of these objects on the luminosity-redshift diagram. The same author studied the luminosity functions of Seifert galaxies and quasars (Terebizh, 1975, 1980).

iv. The spectra of Arakelian galaxies with high surface brightness were obtained by Doroshenko \& Terebizh (1975). A total 40 of 73 galaxies were with emission lines in the spectra. Red shifts and absolute magnitudes of galaxies were determined.

v. The large scale spatial orientation of the major axes of extended double radio sources was studied by Arshakian (1991). It was found the preferable orientation of the axes for 82 objects. Within limits of error the resulting direction was concluded to coincide with the direction of the regular metagalactic magnetic field. vi. The problem of finding the distribution of separation velocities of the components of classical double radio sources were solved by Arshakian $\&$ Andreasian (1993). Most of radio galaxies were found to have velocities in three intervals: $v \approx-0.05 c,-0.015 c$ and $-0.026 c$, where $c$ is the speed of light. For quasars they are in two intervals with average separation velocities of $0.13 c$ and $0.26 c$.

\begin{abstract}
ANNEX
Almost simultaneously with opening the Department of Theoretical Astrophysics in the Byurakan Observatory, the international journal "Astrophysics" was founded. At different times, different members of the department were part of the editorial board of the journal, which soon gained wide recognition from the scientific community. In its turn, the appearance of the journal was an impetus for the development of theoretical astrophysics both in the observatory itself and in the Republic.

The contribution of the members of the Department to the teaching activity and training of scientific personnel in the republic is significant. The broad international cooperation of Department played an important role in the further development of some areas of theory in the observatory, as well as in the creation of new ones.
\end{abstract}




\section{REFERENCES}

V.A. Ambartsumian, The Light Scattering and Absorption in Atmospheres of Planets, Uch. Zap, LGU, No. 82, is. $11,1941$.

V.A. Ambartsumian, New Method for Studying the Light Scattering in the Foggy Medium, Izv. AN SSSR, serie Geogr. I Geophys., No. 3, 97-103, 1942.

V.A. Ambartsumian, On the Scattering of Light by the PlanetaryAtmospheres, Astron. Zh, 19, 30-41, 1942.

V.A. Ambartsumian, On Diffuse Reflection of Light by Turbid Medium, Dokl. Akad. Nauk. SSSR, 38, 257-261, 1943.

V.A. Ambartsumian, On the Problem of Diffuse Reflection of Light, J. Exp. Theoret. Phys., 13, 323-334, 1943.

V.A. Ambartsumian, The Diffusion of Light through Scattering Medium of Large Optical Thickness, Dokl. Akad. Nauk. SSSR, 43, 106-112, 1944.

V.A. Ambartsumian, On the One-Dimensional Case of the Problem of the Scattering and Absorbing Medium of Finite Optical Thickness, Izv. AN ArmSSR, No.1-2, 31-36, 1944.

V.A. Ambartsumian, On the Theory of the Brightness Fluctuations in the Milky Way, Dokl. Akad. Nauk. SSSR, 44, 244-321, 1944.

V.A. Ambartsumian, On the Diffuse Reflection and Transmission of Light by an Anisotropic One-dimensional Scattering Medium of Finite Optical Thickness, Izv. AN ArmSSR, VII, No 5,199-202, 1947.

V.A. Ambartsumian, On the Number of Scatterings of Photons in Foggy Media, Dokl. Akad. Nauk ArmSSR, 8 , 101-105, 1948.

V.A. Ambartsumian, On the Theory of Fluctuations in the Stars Distribution on the Sky, Com. BAO, 2-59, 1951.

V.A. Ambartsumian, Scientific Papers, vol.1, Izd.AN. ArmSSR, Yerevan (1960).

V.A. Ambartsumian, On One Problem of the Non-linear Theory of the Light Scattering in Foggy Medium, Dokl. Akad. Nauk ArmSSR, 38, 225-230, 1964a.

V.A. Ambartsumian, On One Case of Enlightenment of the Medium under the Influence of Radiation, Dokl. Akad. Nauk ArmSSR, 39, 159-165, 1964b.

V.A. Ambartsumian, On the Problem of the Multiple Light Scattering in a Plane-parallel Medium with the Internal Reflection from the Boundary Surface, Trudi Astron. Obs.. LGU, 20, 1964c.

V.A. Ambartsumian, Some Non-linear Problems in the Theory of Radiative Transfer, in: Theory of Stellar Spectra (in Russian), Nauka, Moscow, 225-235, 1966.

V.A. Ambartsumian, Derivation of the Frequency Function of Stellar Flares in a Star Cluster, Astrophysics, 14, 209-217, 1978.

V.A. Ambartsumian, On Some Trends in the Development of Astrophysics, Ann. Rev. Astron. Astrophys. 18, $1-13,1980$.

V.A. Ambartsumian, Scientific Papers, vol.3, Izd.AN.ArmSSR, Yerevan (1988).

V.A. Ambartsumian, On the Fluctuations of the Surface Brightness of Galaxies, Astrophys. Sp, Sci., 144, 635 $-638,1988$, 
V.A. Ambartsumian, in: "A Life in Astrophysics. Selected papers of Viktor Ambartsumian”, Allerton press, NY (1998).

D. Anderson, Variational Principles for the Radiative Transfer Equation of Single Scattering, J. Inst. Math. Applic., 12, 55-62, 1973.

R.R. Andreasian, E.Kh. Danielian, On the Problem of Calculation of the Ambartsumian $\varphi$-Function, Comm. BAO, 50, 114-117, 1978.

R.R. Andreasian, T.G. Arshakian, Radio Luminosities of Pulsar and the Electron Density Distribution in the Galaxy, Astrophysics, 36, 229-234, 1993.

V.Ya. Arsenin, Methods of Mathematical Physics and Special Functions [in Russian], M.,Nauka (1984).

T.G. Arshakian, Large-scale Spatial Orientation of Major Axes of Extendid double Radio Sources, Astrophysics, 35, 394-399, 1991.

T.G. Arshakian, R.R. Andreasian, The Discreteness of the Velocities of Separation of Components of Extended Double Radio Sources, Astrophysics, 36, 235-240, 1993.

G. Batchelor, The Theory of Homogeneous Turbulence, Cambridge Univ. Press, 6th ed., 1970.

M.M. Basko, Frequency Redistribution and Diffusion of Radiation in Resonance X-ray Line, J. Exp. Theoret. Phys, 48, 644-649, 1978.

R. Bellman, R. Kalaba, M. Wing, Invariant Imbedding and Mathematical Physics.I. Particle Processes, J. Math. Phys., 1, 280-308, 1960.

R. Bellman, R. Kalaba, M. Prestrud, Invariant Imbedding and Mathematical Physics, New York, American Elsevier (1963).

R. Bellman, Functional Equations in the Theory of Dynamic Programming.VII., A Partial Differential Equation for the Fredholm Resolvent, Proc. Amer. Math. Soc., 8, 435-440, 1957.

R. Bellman \& G.M. Wing, An Introduction to Invariant Imbedding, New York, Wiley \& Sons (1973).

F.A. Berezin, The Method of Second Quantization, (in Russian), Nauka, M., (1986).

K.M. Case \& P.F. Zweifel, Linear Transport Theory, Reading, MA, Addison-Weslay (1967).

J. Casti, R. Kalaba, Imbedding Methods in Applied Mathematics, (Russian transl.), M., Mir (1976).

S. Chandrasekhar, Radiative Transfer, New York, Dover (1960).

H.S. Chavushian, H. V. Pikichyan, A. V. Oskanian, G. H. Broutian, Reconstruction of The Three-dimensional Density Distribution of Flare Stars in the Pleiades Astrophysics, 47, 313-323, 2004,

L.E. Cram, Multi-Component Models for the Formation of the Chromospheric Ca II K Line. II: The Effect of Velocity Fields, Sol. Phys., 22, 375-386, 1982. E.Kh. Danielian, Radiation Field in a Plane Layer Illuminated by Parallel Rays, Astrophysics, 12, 378-385, 1976.

E.Kh. Danielian, H.V, Pikichian, Radiation Field in a Plane-Parallel Atmosphere Containing Energy Sources, Astrophysics, 13, 147-154, 1977. 
E.Kh. Danielian, Theory of Isotropic Scattering of Radiation in a Homogeneous Plane-Parallel Medium, Astroohysics, 19, 194-202, 1983.

E.Kh. Danielian, On the Problem of Radiation Transfer With Three-demensionality of the Medium, Dokl. Acad. Sci. ArmSSR, 61, 117-123.

V.A. Djrbashian. H.A. Harutyunian, Probability of Photon Scattering by Electrons. The Case of Randomlyly Moving Monoenergetic Electrons, Astrophysics, 22, 228-232, 1985.

V.T. Doroshenko, V.Yu. Terebizh, UBV-Photometry of Seifert Galaxies, Astrophysics, 17, 358-362, 1981.

I.M. Gelfand, S.V. Fomin, Calculus of Variation, Englewood Cliffs, N.J. Prentice-Hall (1965),

I.P. Grant, G.E. Hrant, Solution of Radiative Transfer Problems Using the Invariant $\mathrm{S}_{n}$ Method, Mon. Not. Roy. Astr. Soc., 141, 27-41, 1968.

A.E. Grigorian, T.G. Arshakian, Blue Shift of Supernova Emission Lines Due to Finiteness of the Speed of Light, Astrophysics, 33, 513-517, 1990.

M. Gros \& C. Magnan, The Method of Addition of Layers to Solve the Non-linear Transfer Problem, Astron. Astrophys., 93, 150-154, 1981.

H.A. Harutyunian, A.G. Nikoghossian, The Contours of Spectral Lines for Non-Coherent Scattering, DAN SSSR, 242, 66-69, 1978.

H.A. Harutyunian, A.G. Nikoghossian, Radiation Scattering for a General Law of Frequency Redistribution, $J$. Quantit. Spectrosc. Radiat. Transfer, 19, 135-148, 1978.

H.A. Harutyunian, R.A. Krikorian \& A.G. Nikoghossian, Interpretation of Flares of Red Dwarfs by Means of the Inverse Compton Effect, Astrophysics, 15, 293-300, 1979.

H.A. Harutyunian, A.G. Nikoghossian, The Redistribution Function for the Scattering on Relativistic Electrons, Dokl. Akad. Nauk SSSR, 255, 86-89, 1980.

H.A. Harutyunian, Spectral Line Formation in an Atmosphere with Exponential Distribution of Sources, Dokl. Acad. Sci. ArmSSR, 70, 41, 1980.

H.A. Harutyunian, A Simple Method for Calculation of the Redistribution Function $\mathrm{R}_{I I I}\left(\mathrm{x}^{\prime}, \mathrm{x}\right)$, Comm. of BAO, 52. $137,1980$.

H.A. Harutyunian, A.G. Nikoghossian, The Application of the Invariance Principle to the Problems of Multiple Compton Scattering, Transact. of the All-Union Symp. "The Invariance Principle and Its Applications", 1981, Yerevan, 431-439.

H.A. Harutyunian, A.G. Nikoghossian, The Mean Number of Scatterings for Radiative Transfer with Frequency Redistribution, Dokl. Akad. Nauk SSSR, 268, 1342 - 1346, 1983.

H.A. Harutyunian, Reflection from the Semi-infinite Inhomogeneous Atmosphere for the General Laws of Noncoherent Scattering, Astrophysics, 23, 584-590, 1985.

H.A. Harutyunian, A.G. Nikoghossian, Statistical Description of Radiation Field on the Basis of the Invariance Principle. IV., The Results of Numerical Calculations, Astrophysics, 27, 536-543, 1987.

H.A. Harutyunian, V.A. Djrbashian, A.G. Nikoghossian, Energy Losses for the Photon Scattering by Free Electrons, Astrophysics, 29, 119-131, 1988. 
H.A. Harutyunian, A.G. Nikoghossian, The Application of the Invariance Principle to the Multiple Compton Scattering Problem, in: Principle of Invariance and its Applications, Eds. M.A. Mnatsakanian \& O.V. Pikichyan, 431-439, Izd. AN ArmSSR (in Russian), 1989.

H.A. Harutyunian, Formation of Spectral Lines in the Medium of Finite Thickness for Non-Coherent Scattering of Radiation, Dokl. Acad. Nauk SSSR, 320, 1073-1077, 1991.

H.A. Harutyunian, Bilinear Expansion of the Frequency Redistribution Functions, 1991, Dokl. Acad. Nauk SSSR, 321, 285-289, 1991.

H.A. Harutyunian, Radiative Transfer for Incoherent Scattering in a Semi-Infinite Atmosphere, Containing Energy Sources, Astrophysics, 36, 335-342, 1993.

H.A. Harutyunian, The Expansion of the Voigt Function, Astrophysics, 39, 370-374, 1996.

H.A. Harutyunian, Scattering of Radiation by Free Electrons. The Angle-averaged Redistribution Function, Astrophysics, 49, 405-414, 2006.

H.A. Harutyunian \& G. Alecian, Radiation Transfer in an Inhomogeneous Medium. Optical Depth Depending Absorption Coefficient, Astrophysics, 55, 251-260, 2012.

H.A. Harutyunian, G.Alecian, K.G. Khachatryan, A.V. Vardanyan, Bilinear Expansion for Redistribution Functions, J. Quantit. Spectrosc. Radiat. Transfer, 189, 64-69, 2016.

H.A. Harutyunian, H.A. Poghosyan Numerical Solution of the Ambartsumian Functional Equations for a Plane Medium with Finite Thickness, Astrophysics, 61, 525-539, 2018.

V.S. Hayrapetian, A.G. Nikoghossian, Effect of the Plasma Turbulence in the Atmospheres of Active Stars, Astrophysics, 29, 455-462, 1988.

V.S. Hayrapetian, V.V. Vikhrev, A.G. Nikoghossian, On the Generation of Magnetic Field in the Floating Strength Magnetic Tubes, DAN Arm. SSR,87, 171-173, 1989.

Hubeny, Probabilistic Interpretation of Radiative Transfer, I,II, Astron. Astrophys., 185, 332, 1987a, 185, 336, $1987 b$.

Hubeny, in: Atmospheres of Early Type Stars, U. Huber, U.S. Jeffery, ed., Lecture Notes in Physics, No. 401, p. 377, Berlin, Springer, 1982.

D.G. Hummer, Non-coherent scattering: I. The redistribution function with Doppler broadening. Mon. Not. Roy Astron. Soc., 125, 21-37, 1962.

A.F. Illarionov, R.A. Sunyaev, Compton Scattering by Thermal Electrons in X-Ray Sources, Astron. Zh., 49, 581-587, 1972.

G.L. Israelian, M. De Groot, Forbidden Lines in the Spectrum of P Cyg, Astrophysics, 34, 171-174, 1992.

G.L. Israelian, A.G. Nikoghossian, The Spectral Variations of P Cyg, Astrophysics, 36, 119-121, 1993.

G.L. Israelian, L.V. Tambovtseva, A.G. Nikoghossian, Wind Dynamics of LBV-Stars, Astron. Zh, 70, 976-983, 1993.

G.L. Israelian, A.G. Nikoghossian, On Schuster's Mechanism of Emission Line Formation, J. Quantit. Spectrosc. Radiat. Transfer, 56, 509-512, 1996. 
V.V. Ivanov, Nonlinear Equations in Linear Problems of Radiative Transfer in Plane Atmospheres, Astron. Zh., 22, 612-618, 1978.

V.V. Ivanov, Radiative Transfer and the Spectra of Celestial Objects (in Russian), M., Nauka, (1969).

J.T. Jefferies, C. Lindsey, Radiative Transfer in Inhomogeneous Atmospheres, A Statistical Approach, Astrophys. J., 335, 372-382, 1988.

I.D. Karachentsev, V.Yu. Terebizh, On the Statistics of "Interacting” Galaxies, Astrophysics, 4, 443-449, 1968.

S. Kichenessamy, R.A. Krikorian, A.G. Nikoghossian, The Relativistic Doppler Broadening of the Line Absorption Profile, J. Quantit. Spectrosc. Radiat. Transfer, 27, 653-655, 1982.

M.G. Krein, On New Method of Solution of Linear Integral Equations of the First and the Second Kinds, Dokl. Acad, Sci. SSSR, 100, 413-416, 1955.

R.A. Krikorian, A.G. Nikoghossian, On the Lagrangian Formalism in the Radiative Transfer Theory, J. Quantit. Spectrosc. Radiat. Transfer, 56, 465-469, 1996.

S. Koutchmy. Some Morphological Pecularities of the Solar Corona on 22 September 1968, Astrophys. Let., 3, 215-2020, 1969

S. Koutchmy, A.G. Nikoghossian, Coronal Linear Threads: W-L Radiation of Supra-thermal Streams, Astron. Astrophys., 395, 983-989, 2002.

S. Koutchmy, A.G. Nikoghossian, Analysis of the Radiation of Coronal Suprathermal Streams, Astrophysics, $\mathbf{4 8}$, 62-67, 2005.

W. Magnus, Comm. Pure and Appl. Math., VII (??), 649, 1954.

C. Magnan, The Method of Addition of Layers for Non-linear Radiative Transfer Problems: practical applications, Astron. Astrophys., 543-551, 1993.

E. Menkohn, S. Richling, Radiative Transfer with Finite Elements.II.Ly-alpha Line Transfer in Moving Transfer, Astron. Astrophys., 392, 827-880, 2002.

D. Mihalas, Stellar Atmospheres, Freeman \& Co, San Francisco (1970).

I.N. Minin, On the Theory of the Radiation Diffusion in a Semi-infinite Medium, Dokl. Akad. Nauk. SSSR, 120, 63-65, 1958.

M.A. Mnatsakanian, Quasiasymptotic Solutions of the Problem of Radiation in a Slab of Finite Thickness, I. Astrophysics, 11, 434-447, 1975.

M.A. Mnatsakanian, Quasiasymptotic Solutions of the Problem of Radiation in a Slab of Finite Thickness, II. Astrophysics, 12, 295-311, 1976.

R.A. Muradian, H.V. Pikichyan, The Possible Reduction of Dome Impact on Images of Stars, CommBAO, 58, 100-107, 1986.

A.G. Nikoghossian, Non-Linear Problem of Polychromatic Diffuse Reflection from an Infinitely Deep OneDimensional Medium, Dokl. Akad. Nauk ArmSSR, 39, 227-233, 1964.

A.G. Nikoghossian, Polychromatic Diffuse Reflection of Light from an Infinitely Deep One-Dimensional Medium, Astrophysics, 1, 285-296, 1965. 
A.G. Nikoghossian, H.A. Haruthyunian, Spectral Lines Formation for a General Law of Redistribution, Dokl. Akad. Nauk SSSR, 229, 583-586, 1976.

A.G. Nikoghossian, The Problem of Diffuse Reflection for Redistribution of Radiation over Frequencies and Directions, Dokl. Akad. Nauk SSSR, 235, 786 -789, 1977.

A.G. Nikoghossian, H.A. Haruthyunian, Diffuse Reflection of Light in the Case of an Arbitrary Phase Function,Astrophysics, 14, 223-230, 1978.

A.G. Nikoghossian, H.A. Haruthyunian, The Radiation Field in a Semi-Infinite Atmosphere Containing Energy Sources, Astrophys. Sp. Sci., 64, 285 -299, 1979.

A.G. Nikoghossian, Diffuse Reflection of Light from a Semi-Infinite Atmosphere for the Redistribution Law $\mathrm{r}_{I I}(\mathrm{x}, \mathrm{X} \prime, \gamma)$, Dokl. AN Arm.SSR, 68, 176-182, 1979.

A.G. Nikoghossian, Statistical Description of Radiation Field on the Basis of the Invariance Principle I., The Mean Number of Scatterings in a Medium Illuminated from Outside, Astrophysics, 21, 527-537, 1984.

A.G. Nikoghossian, Statistical Description of Radiation Field on the Basis of the Invariance Principle.II., The Mean Number of Scatterings in a Medium Containing Energy Sources, Astrophysics, 21, 685-693, 1984.

A.G. Nikoghossian, "Reflection" Function for an Infinite Atmosphere in the Case of Non-Coherent Scattering, Astrophysics, 1986, 25, 596-602, 1986.

A.G. Nikoghossian, Statistical Description of Radiation Field on the Basis of the Invariance Principle.III., Average Time of Photon Travel in the Scattering Medium, Astrophysics, 24, 89-99, 1986.

A.G. Nikoghossian, On the Problem of the Formation of Quasars Emission Lines by the Cherenkov Mechanism, Astrophysics, 37, 247-255, 1994.

A.G. Nikoghossian, Some New Nonlinear Relations of the Radiative Transfer Theory, Astrophysics, 38, 577-586, 1995.

A.G. Nikoghossian, G.L. Israelian, The Effect of Strong Scattering Processes in the Continuum on the Stellar Emergent Energy Distribution, J. Quantit. Spectrosc. Radiat. Transfer, 56, 501-507, 1996.

A.G. Nikoghossian, Invariance Principle and Bilinear Relations of the Radiative Transfer Astrophys. J., 483, 849-856, 1997.

A.G. Nikoghossian, S. Pojoga, Z. Mouradian, On the Radiative Transfer in Atmospheres with Randomly Distributed Inhomogeneities, Astron. Astrophys, 325, 813-818,1997.

A.G. Nikoghossian, S. Pojoga, Z. Mouradian A Statistical Approach to the Investigation of Fine Structure of Solar Prominences, Astron. Astrophys., 332, 325-338, 1998.

A.G. Nikoghossian, S. Pojoga, Z. Mouradian, A Statistical Investigation of Prominence Fine Structure in the EUV, In: IAU Coll. 167, in: New Perspectives on Solar Prominences, (Eds. Webb D., Shmieder. B. \& Rust. D.), $59-65,1998$.

A.G. Nikoghossian, S. Pojoga, Z. Mouradian, Statistical Characteristics of Radiation Formed in Atmosphere with Randomly Distributed Inhomogeneities, Astron. Astrophys, 342, 785-798, 1999.

A.G. Nikoghossian, The Variational Formalism and Bilinear Relations of the Radiative Transfer Theory. J. Quantit, Specrosc. Radiat. Transfer, 61, 345-359, 1999. 
A.G. Nikoghossian, Z. Mouradian, Profiles of the Spectral Lines Formed in Stochastic Multicomponent Atmosphere, Astron. Astrophys, 360, 1086-1095, 2000.

A.G. Nikoghossian, Non-Linear Relations for Statistical Mean Quantities Describing the Multiple Scattering Process, Astrophysics, 43, 337-342, 2000.

A.G. Nikoghossian, On a Covering Problem and its Astrophysical Application, Astrophysics, 44, 131-136, 2001.

A.G. Nikoghossian, S. Koutchmy, On Interpretation of the Radiation of Coronal Suprathermal Streams. I, Astrophysics, 44, 528-535, 2001.

A.G. Nikoghossian, S. Koutchmy On Interpretation of the Radiation of Coronal Suprathermal Streams.II., Astrophysics, 45, 489-496, 2002.

A.G. Nikoghossian, Intensity Fluctuations of Radiation Escaping from a Multicomponent Stochastic Atmosphere. I,, Astrophysics, 45, 223-231, 2002.

A.G. Nikogohossian, Radiative Transfer in One-dimensional Inhomogeneous Atmospheres Astron. Astrophys., 422, 1059 -1066, 2004.

A.G. Nikoghossian, Radiative Transfer in Inhomogeneous Atmosphere, I, II, III, Astrophisics, 47, 104-115,2004a, 248-259, 2004b..412-420, 2004c.

A.G. Nikoghossian, J. Aboudarham, Z. Mouradian, Fluctuations of the Radiation Intensity Outgoing from a Multicomponent Stochastic Atmosphere. II, Astrophysics, 48, 253 - 261, 2005.

A.G. Nikoghossian, Spectral Line Formation in a Dynamically Active Multicomponent and Stochastic Atmosphere, Astrophysics, 50, 94 - 104, 2007a.

A.G. Nikoghossian, Spectral Line Formation in a Mesoturbulent Atmosphere, Astrophysics, 50, 175-186, $2007 \mathrm{~b}$.

A.G. Nikoghossian, Diffuse Reflection of the Line-radiation from a Semi-infinite Turbulent Atmosphere, Astrophysics, 50, 321-332, 2007c.

A.G. Nikoghossian, Ambartsumian's Methods in the Radiative Transfer Theory, 52, 1 - 23, 2009a.

A.G. Nikoghossian, Ambartsumian's Principle of Invariance and Some Non-linear Relations of the Radiative Transfer Theory, Astrophysics, 52, 431-439, 2009b.

A.G. Nikogohossian, Z. Mouradian, On Determining the Microturbulent Velocities of Solar Prominences, Astrophysics, 53, 387-395, 2010.

A.G. Nikogohossian, Group-theoretical Description of Radiation Transfer in One-dimensional Medium, Astrophysics, 54, 126-138, 2011a.

A.G. Nikogohossian, Bilinear Integrals of the Radiative Transfer Equation, Astrophysics, 54, 242-249, 2011 b.

A.G. Nikogohossian, Solution of Linear Problems of the Radiation Transfer in Plane-parallel Atmosphere. I, Astrophysics, 54, 553-567, 2011c.

A.G. Nikogohossian, Solution of Linear Problems of the Radiation Transfer in Plane-parallel Atmosphere. II., Astrophysics, 55, 261-274, 2012a.

A.G. Nikoghossian, Ambartsumian's Methods in the Theory of Radiative Transfer, in: Ambartsumian's Legacy and Active Universe, Springer, 37-59, $2012 \mathrm{~b}$. 
A.G. Nikoghossian, Solution of Linear Problems of the Radiation Transfer in Plane-parallel Atmosphere. III., Astrophysics, 56, 130-141, 2013a.

A.G. Nikogohossian, On Some Trends in the Progress of Astrophysical Radiative Transfer, Light Scat. Rev., 8 , 377-426, 2013b.

A.G. Nikoghossian, On the Role of the Reflection Function in Describing the Diffusion in the Plane-parallel Inhomogeneous Atmosphere, Astrophysics, 56, 553-560, 2013c.

A.G. Nikoghossian, Groups and their Representations in the Radiative Transfer Theory. I,II., Astrophysics, 57, 272-286, 2014a, 375-383, 2014b.

A.G. Nikoghossian, Groups in the Radiative Transfer Theory, J. Quantit. Spectrosc. Radiat. Transfer, 183, 90-99, 2016.

A.G. Nikoghossian, Radiation Diffusion in an Inhomogeneous Turbulent Atmosphere, Astrophysics, 60, 380-392, 2017.

A.G. Nikoghossian, Groups and their Representations in the Radiative Transfer Theory. III, Astrophysics, 62, 92-117, 2019.

A.G. Nikoghossian, Investigation of Some Manifestations of Non-Stationarity in the Spectra of the Early Type Supergiants, Astrophysics, 63, 2020 (in print).

V.S. Oskanian, V.Yu. Terebizh, Some Characteristics of the Flare Activity of UV Ceti Type Stars, 7, 48-54, 1971a, 287-294, 1971b.

A. Peraiah, Principles of Invariance in Radiative Transfer, Sp. Sci. Rev., 87, 465 - 538, 1999.

H.V. Pikichyan, Green's Function of a Plane-parallel Layer in the Case of Incoherent Anisotropic Scattering, Astrophysics, 14, 96-108, 1978a,

H.V. Pikichyan, The Problem of Diffuse Reflection for an Arbitrary Law of the Frequency Redistribution, Dokl. Acad. Sci. ArmSSR, 67, 151-156, 1978b.

H.V. Pikichyan, Green's Function of an Optically Thick Layer, Astrophysics, 16, 209-217, 1980 ,

H.V. Pikichyan, Determination of the Radiation Field in a Composite Medium by Known Solutions of the Transfer Problems for Arbitrarily Chosen macro-Parts, Dokl. Acad. Sci. SSSR, 263, 601-606, 1982a.

H.V. Pikichyan. General Relations of Invariance in Investigating the Transfer Problems fot the Media of Arbitrary Geometrical and Physical Characteristics, Dokl. Acad. Sci. SSSR, 262, 860-863, 1982b.

H.V. Pikichyan, Solution of the Transfer Problem in an Isolated Part of the Volume by the Solution Known for the Whole Volume, Dokl. Acad. Sci. SSSR, 273, 861-865, 1983.

H.V. Pikichyan, Invariance Principle in the Problems of Addition of Layers of Arbitrary Properties, CommBAO, 55, 5-16, 1984.

H.V. Pikichyan, Nonlinear Diffuse Reflection and Transmission of Radiative Energy by a Layer of Finite Thickness, Astrophysics, 53, 251-267, 2010.

H.V. Pikichyan, On the linear properties of the nonlinear radiative transfer problem. J. Quantit. Spectrosc. Rad. Transfer., 183, 113-127, 2016a. 
H.V. Pikichyan, Internal Radiation Field in the Nonlinear Transfer Problem for a One-dimensional Anisotropic Medium. I.II, Astrophysics, 59, 114-125, 2016b, 272-284, 2016c.

H.V. Pikichyan, The Inverse Problem of Statistical Determination of the Total Number, Luminosity Function, and Brightness Variability Characteristics of T Tau Type Stars in Stellar Aggregates, Astrophysics, 61, 333-346, 2018.

H.V. Pikichyan, Analytical Solution of the Nonlinear Problem of Radiation Diffusion in a Pure Scattering Medium, 62, 387-401, 2019.

R.W. Preisendorfer, Hydrologic Optics, vol. IV, U.S. Dept. of Commerse, Honolulu, Hawaii (1976).

R. Redheffer, On the Relation of Transmission-line Theory to Scattering and Transfer, J. Math.E Phys., 41, 1-41, 1962.

G.B. Rybicki, Integrals of the Transfer Equations, Astrophys. J., 213, 165-176, 1977.

G.B. Rybicki, in: Methods on Radiative Transfer, W. Kalkofen, ed., 21-64, Cambridge, Cambridje Univ. Press. 1984.

M. Scott, Invariant Imbedding and its Applications to Ordinary Differential Equations. An Introduction, Reading, MA. Addison-Wesley (1973).

Secchi-Pestelini, L. Barletti, Radiative Transfer in Stochastic Universe. .I, New Astron., 6, 151-163, 2001.

V.V. Sobolev, Light Scattering in Inhomogeneous Atmosphere, Dokl. Acad. Sci. SSSR, 111, 1000-1003, 1956.

V.V. Sobolev, Radiation Diffusion in a Semi-infinite Medium, Dokl. Acad, Sci. SSSR, 116, 45-48, 1957.

V.V. Sobolev, On the Theory of Radiation Transfer in Stellar Atmospheres, Astron. Zh., 36, 573-578, 1959.

V.V. Sobolev, A Treatise on Radiative Transfer, Princeton: Van Nostrand (1963).

V.V. Sobolev, Course in Theoretical Astrophysics, (in Russian), M., Nauka (1985).

E. Tandberg-Hanssen, The Nature of Solar Prominences, Kluver, Dordrecht (1995).

M. Tavel, Application of Noether's Theorem to the Transport Equation, Transport Theory Statist. Phys., 1, 271$285,1971$.

V.Yu. Terebizh, Some Nonlinear Problems in the Theory of Radiative Transfer within Spectral Lines, Astrophysics, 3, 129-132, 1967.

V.Yu. Terebizh, polychromatic Light Scattering in a Semi-infinite Medium, 5, 359-370, 1969.

V.Yu. Terebizh, Softening of Radiation by Multiple Compton Scattering, 6, 368-371, 1970.

V.Yu. Terebizh, Source Number as a Function of the Flux and the V/ $\mathrm{V}_{m}$ Method in the Investigation of the Evolution of the Quasi-stellar Radio Sources, Astrophysics, 9, 118-124, 1973.

V.Yu. Terebizh, Luminosity Function of Quasi-stellar Radio Sources, Astrophysics, 11, 104-105, 1975.

V.Yu. Terebizh, Luminosity Function of Seyfert Galaxies, Astrophysics, 16, 36-46, 1980.

L.H. Thomas, The Slow Contraction or Expansion of a Fluid Sphere, Mon. Not. Roy. Astron. Sci., 91, 122-127, 1930. 
M.M. Vainberg, Variational Methods for the Study of Non-Linear Operators, san Francisco, Holden Day (1964).

H.C. Van de Hulst, Light scattering Tables, Formulas and Applications, Vol.1, New York, Academic Press (1980).

S. Vsessviatski \& E. Bougoslavskaya, The Solar Eclipse of 1936 June 19, Mon. Not. Roy. Astr. Soc., 104, 140-148, 1944.

E. Wigner, Group Theory [Russian translation], izd. IL, Moscow (1961).

E.G. Yanovitskij, Light Scattering in Inhomogeneous Atmospheres, New York, Springer (1997).

N.B. Yengibarian, A.G. Nikoghossian, Diffuse Reflection of Quanta from the Turbulent Medium, In: Stars, Nebulas, Galaxies,: Izd. Acad. Sci. Arm.SSR, Yerevan, pp.65-72 (1969).

N.B. Yengibarian, A.G. Nikoghossian, Incoherent Scattering. II, III., Astrophysics, 8, 39-51, 1972a, 128-136, 1972b.

N.B. Yengibarian, A.G. Nikoghossian, Diffuse Reflection of the Resonant Radiation from a Semi-Infinite Medium, Dokl. ANArmSSR, 54, 91-95, 1972c.

N.B. Yengibarian, On Factoriization of the Symmetric Integral Operators, Dokl. Akad. Nauk SSSR, 203, 4-8, 1972.

N.B. Yengibarian, A.G. Nikoghossian, Incoherent Scattering. IV. Infinite Medium, Astrophysics. 9, 41-50, 1973.

N.B. Yengibarian, A.G. Nikoghossian, Incoherent Scattering, J. Quantit. Spectrosc. Radiat. Transfer, 13, $787-$ $811,1973$.

N.B. Yengibarian, M.A. Mnatsakanian, Dokl. Akad. Nauk. SSSR, 217, 106, 1974.

N.B. Yengibarian, A.G. Nikoghossian, M.S. Gevorkian, Incoherent Scattering. V. Astrophysics., 11, 303-315, 1975.

Ya.B. Zel'dovich, R.A. Sunyaev, Astrophys. Space Sci., 4, 301 (1969).

V.S. Hayrapetian, A.G. Nikoghossian, The Linear Z-Pinch and Stellar Flare Phenomena, Astrophysics, 30, 329336, 1989.

V.Yu. Terebizh, Non-stationary Diffusion of Radiation in a Gas, Astrophysics, 4, 45-46, 1968. 